\title{
Nested sums of symbols and renormalised multiple zeta values
}

\author{
Dominique MANCHON, Sylvie PAYCHA
}

February 2, 2011

\begin{abstract}
We define renormalised nested sums of symbols on $\mathbb{R}$ which satisfy stuffle relations. For appropriate symbols these give rise to renormalised Euler-Zagier-Hoffman multiple zeta (and Hurwitz zeta) functions which satisfy stuffle relations at all arguments. We show the rationality of renormalised multiple zeta values at nonpositive integer arguments. These results generalise to radial symbols on $\mathbb{R}^{n}$ giving rise to a higherdimensional analog of multiple zeta functions.
\end{abstract}

\section{Acknowledgements}

The second author would like to thank Pierre Cartier for interesting discussions around multiple zeta functions as well as Steven Rosenberg for pointing out reference GSW. Thanks to both of them for giving the second author the opportunity to present part of the results at a preliminary stage of their development. The first author thanks Kurusch Ebrahimi-Fard and Michael E. Hoffman for crucial references and details about quasi-shuffle products, and Herbert Gangl for checking the table at the end of section 4 with a PARI routine. Both authors are indebted to Li Guo for various interesting discussions and for bringing to our attention and commenting for us his own work with Bin Zhang [GZ] which also uses a Birkhoff factorisation to renormalise multiple zeta functions. We also greatly appreciate comments sent by Shlomo Sternberg and Hoang Ngoc Minh.

The second author also thanks the Max Planck Institute in Bonn where parts of this paper were completed; she is specially grateful to Bin Zhang for discussions during her stay at the Max Planck Institute in the summer 2006, which were essential to clarify some fundamental points in the paper.

We thank the referees for their numerous and very helpful comments and suggestions which greatly contributed in improving the final presentation. 


\section{Introduction}

Multiple zeta function: 1

$$
\zeta\left(s_{1}, \ldots, s_{k}\right):=\sum_{0<n_{k}<\cdots<n_{1}} \frac{1}{n_{1}^{s_{1}}} \cdots \frac{1}{n_{k}^{s_{k}}}
$$

converge when $\sum_{j=1}^{m} \operatorname{Re} s_{j}>m$ for all $m \in\{1, \ldots, k\}$ (see [G] Theorem 2.25, [Zh] and can be meromorphically extended to $\mathbb{C}^{k}$, with singularities located at:

$$
\begin{aligned}
s_{1} & =1, \\
s_{1}+s_{2} & =2,1,0,-2,-4,-6, \ldots, \\
s_{1}+\cdots+s_{j} & \in \mathbb{Z} \cap]-\infty, j], j=3,4, \ldots, k
\end{aligned}
$$

( $\mathrm{AET}$, see also [Zh] and [G] Theorem 2.25). They satisfy quasi-shuffle (or "stuffle") relations on the convergence domain 3 , an example of which reads:

$$
\zeta\left(s_{1}\right) \zeta\left(s_{2}\right)=\zeta\left(s_{1}, s_{2}\right)+\zeta\left(s_{2}, s_{1}\right)+\zeta\left(s_{1}+s_{2}\right) .
$$

The aim of this article is threefold:

- to extend multiple zeta functions to all arguments $s_{i}$ using a renormalisation procedure à la Connes and Kreimer, in such a way that the renormalised values satisfy stuffle relations,

- to prove the rationality of multiple zeta values at nonpositive integer arguments $\left(s_{1}, \ldots, s_{k}\right)$, and the very much related holomorphicity of the maps $z \mapsto \zeta\left(s_{1}-\right.$ $\left.c_{1} z, \ldots, s_{k}-c_{k} z\right)$ for complex numbers $c_{1}, \ldots, c_{k}$ with positive real part.

- to provide natural higher dimensional analogs of multiple zeta functions which we renormalise in a similar manner.

In our approach, the requirement that multiple zeta functions should also obey stuffle relations at all arguments boils down to requiring that certain maps define characters on Hopf algebras given by the tensor product algebras of classical symbols with constant coefficients (see paragraph1.1.1 for the definition) equipped with the stuffle product 4 . Let $\chi$ be a smooth cut-off function which vanishes around the origin and such that $|\xi| \geq 1 \Rightarrow \chi(\xi)=1$, and consider the functions $\sigma_{s}(\xi):=\chi(\xi)|\xi|^{-s}$, which are elementary examples of classical symbols. It was shown in $\mathrm{MP}$ that, as $N \rightarrow+\infty$, for any $\left(s_{1}, \ldots, s_{k}\right) \in \mathbb{C}^{k}$ the sum:

$$
\sum_{0<n_{k}<\cdots<n_{1} \leq N} \sigma_{s_{1}}\left(n_{1}\right) \cdots \sigma_{s_{k}}\left(n_{k}\right)
$$

\footnotetext{
${ }^{1}$ also known under the purely greek-rooted expression "polyzeta functions" (P. Cartier). We refer the reader to e.g. [H],$[\mathrm{Z},[\mathrm{W}],[\mathrm{ENR}$, CEMP, [Mi], Zu] among a long list of articles on algebraic relations obeyed by multiple zeta functions.

${ }^{2}$ In our opinion, there is a misprint in both references concerning this convergence condition.

${ }^{3}$ We shall not consider shuffle relations and regularisation relations for multiple zeta values, which, at least to our knowledge, only make sense at positive integer arguments.

${ }^{4}$ In [GZ, the authors have a similar approach using a Birkhoff factorisation to renormalise multiple zeta functions. Our construction should relate to theirs via a Mellin transform.
} 
admits an asymptotic expansion in powers of $N$ and $\log N$, as does the iterated integral:

$$
\int_{r \leq x_{k} \leq \cdots \leq x_{1} \leq N} \sigma_{s_{1}}\left(x_{1}\right) \cdots \sigma_{s_{k}}\left(x_{k}\right) d x_{1} \cdots d x_{k}
$$

(for some $r>0$ ) to which the above sum is related via an iterated use of the EulerMacLaurin formula. We define the cut-off nested sum and the cut-off nested integral of the tensor product $\sigma_{s_{1}} \otimes \cdots \otimes \sigma_{s_{k}}$ as the respective constant terms in $N$, which we denote by

$$
\sum_{<}^{\text {Chen }} \sigma_{s_{1}} \otimes \cdots \otimes \sigma_{s_{k}} \quad \text { and } \quad f_{r}^{\text {Chen }} \sigma_{s_{1}} \otimes \cdots \otimes \sigma_{s_{k}}
$$

respectively, in honour of K.T. Chen Ch. When $\operatorname{Re}\left(s_{1}+\cdots+s_{m}\right)>m$ for all $m$ in $\{1, \ldots, k\}$, then the above cut-off nested integral and sum both converge and coincide with the ordinary nested integral and sum respectively, which obey shuffle, resp. stuffle relations. The nested sum is nothing but $\zeta\left(s_{1}, \ldots, s_{k}\right)$ in this case. However these shuffle and stuffle properties do not extend in a straightforward manner to cut-off integrals and sums; nevertheless, a holomorphic regularisation of the symbols (which perturbs their order) provides shuffle, resp. stuffle relations by analytic continuation as identities of meromorphic maps. These constructions lead to algebra morphisms $\Phi_{r}^{\mathcal{R}}$ (see Proposition 7 ) and $\Psi^{\mathcal{R}}$, (see Proposition 12) defined on Hopf algebras $\mathcal{H}_{0}$ and $\mathcal{H}$ respectively, with values in meromorphic functions, where $\mathcal{H}_{0}$ (resp. $\mathcal{H}$ ) is the tensor algebra built on the space of classical symbols on $\mathbb{R}$ (or a suitable subalgebra of it) equipped with the shuffle product (resp. a suitable stuffle product) and deconcatenation coproduct.

Let us make this statement more precise: on the one hand, a holomorphic regularisation $\mathcal{R}: \sigma \mapsto \sigma(z)$ (e.g. Riesz or dimensional regularisation, see section 1.5) extends multiplicatively to a regularisation $\tilde{\mathcal{R}}$ on the tensor algebra of classical symbols defined by

$$
\tilde{\mathcal{R}}\left(\sigma_{1} \otimes \cdots \otimes \sigma_{k}\right):=\mathcal{R}\left(\sigma_{1}\right) \otimes \cdots \otimes \mathcal{R}\left(\sigma_{k}\right),
$$

leading to a meromorphic map

$$
\Phi_{r}^{\mathcal{R}}\left(\sigma_{1} \otimes \cdots \otimes \sigma_{k}\right): z \mapsto f_{r}^{\text {Chen }} \tilde{\mathcal{R}}\left(\sigma_{1} \otimes \cdots \otimes \sigma_{k}\right)(z),
$$

with poles of order $\leq k$. The regularisation $\tilde{\mathcal{R}}$ is compatible with the shuffle product:

$$
\tilde{\mathcal{R}}(\sigma \amalg \tau)=\tilde{\mathcal{R}}(\sigma) \amalg \tilde{\mathcal{R}}(\tau) .
$$

On the other hand, one can extend a regularisation $\mathcal{R}$ on classical symbols to a regularisation $\tilde{\mathcal{R}}^{\star}$ on the tensor algebra of classical symbols which is compatible with the above mentioned stuffle product $\star$, i.e. such that

$$
\tilde{\mathcal{R}}^{\star}(\sigma \star \tau)=\tilde{\mathcal{R}}^{\star}(\sigma) \star \tilde{\mathcal{R}}^{\star}(\tau) .
$$

by "twisting" the regularisation $\tilde{\mathcal{R}}$ by the Hoffman Hopf algebra isomorphism from $\mathcal{H}_{0}$ to $\mathcal{H}$ (see section 3.1 and [H2]). This leads to a meromorphic map

$$
\Psi^{\mathcal{R}}\left(\sigma_{1} \otimes \cdots \otimes \sigma_{k}\right): z \longmapsto \sum_{<}^{\text {Chen }} \tilde{\mathcal{R}}^{\star}\left(\sigma_{1} \otimes \cdots \otimes \sigma_{k}\right)(z) .
$$


The meromorphicity property for cut-off iterated integrals and the upper bound on the order of the poles follow from properties of cut-off integrals of holomorphic families of log-polyhomogeneous symbols, a natural generalisation of classical symbols which includes positive integer powers of logarithms (see paragraph 1.1.1 and [L]). Similar meromorphicity properties and a similar pole structure hold for cut-off Chen sums (see section 3.2), which can be derived from the corresponding properties of cut-off Chen integrals via the Euler-MacLaurin formula. We prove this way (Proposition 6) that the maps

$$
\left(z_{1}, \ldots, z_{k}\right) \mapsto \sum_{<}^{\text {Chen }} \mathcal{R}\left(\sigma_{1}\right)\left(z_{1}\right) \otimes \cdots \otimes \mathcal{R}\left(\sigma_{k}\right)\left(z_{k}\right)
$$

are meromorphic with poles on a discrete set of hyperplanes

$$
q \sum_{i=1}^{j} z_{i} \in-\sum_{i=1}^{j} \alpha_{i}(0)+[-j, \infty[\cap \mathbb{Z}
$$

in the case when $\alpha_{i}(z)=\alpha_{i}(0)+q z$ is the holomorphic order of the symbol $\sigma_{i}(z)$. When $\sigma_{j}(z)(\xi)=\chi(\xi)|\xi|^{-z}$ (and hence $\alpha_{i}(z)=-z$ ), we recover (Corollary 国) the pole structure $\left.\left.\sum_{i=1}^{j} z_{i} \in\right]-\infty, j\right] \cap \mathbb{Z}$ of multiple zeta functions recalled at the beginning of the introduction, up to the specificities in depths one and two which are discussed later in the paper (Theorem 9).

The Birkhoff factorisation of these characters (see Theorems 2] 17) then provides renormalised values at $z=0$ for any tensor product $\sigma_{1} \otimes \cdots \otimes \sigma_{k}$ of classical symbols, which obey the same shuffle, resp. stuffle relations. Applying this to $\sigma_{i}(\xi)=|\xi|^{-s_{i}} \chi(\xi)$ and a regularisation procedure $\mathcal{R}_{\mu}\left(\sigma_{i}\right)(z)=\chi(\xi)|\xi|^{-s_{i}-z-\mu z^{2}} \quad(\mu \in \mathbb{R})$, leads on the one hand to renormalised continuous analogs of multiple zeta functions (see Corollary 1) which obey the expected shuffle relations and on the other hand to renormalised multiple zeta values $\zeta^{\mathcal{R}}\left(s_{1}, \ldots, s_{k}\right)$ (depending on the regularisation procedure $\mathcal{R}$ ) at all arguments $s_{i}$, which indeed verify the stuffle relations. Multiple zeta functions are sometimes considered in their "weak inequality version":

$$
\bar{\zeta}\left(s_{1}, \ldots, s_{k}\right):=\sum_{0<n_{k} \leq \cdots \leq n_{1}} \frac{1}{n_{1}^{s_{1}}} \cdots \frac{1}{n_{k}^{s_{k}}},
$$

which are simultaneously renormalised along the same lines.

We show that renormalised multiple zeta functions $\bar{\zeta}\left(s_{1}, \ldots, s_{k}\right)$ and $\zeta\left(s_{1}, \ldots, s_{k}\right)$ at non-positive integer arguments are rational numbers which do not depend on the regularisation chosen, so that we can drop the superscript $\mathcal{R}$ in this case (Theorem 10). This is due to the holomorphicity of the maps $z \mapsto \zeta\left(s_{1}+c_{1} z, \ldots, s_{k}+c_{k} z\right)$ at these arguments which holds for any complex numbers $c_{1}, \ldots, c_{k}$. Li Guo and Bin Zhang GZ have proposed another construction for stuffle-compatible multiple zeta values. Their construction however makes sense only when all the arguments have the same sign (positive or negative). They also use Birkhoff decomposition in an essential way, but starting from a different Hopf algebra, and the values are rational numbers at non-positive arguments as well. 
Proposals for multiple zeta values at nonpositive arguments have already been formulated (see e.g. G, AET]) but, ref. GZ excepted, without taking quasi-shuffle relations into account. Let us also mention the work of Jean Ecalle $\mathrm{E}$. on multiple zeta functions, in which a brief discussion of values at nonpositive arguments is outlined, in the language of mould calculus.

We give an explicit formula (in terms of Bernoulli numbers) for double zeta values (equation (77)). Our double zeta values at nonpositive arguments turn out to coincide with those proposed by S. Akiyama, S. Egami and Y. Tanigawa at the end of their paper as:

$$
\zeta\left(-a_{1},-a_{2}\right)=\lim _{z \rightarrow 0} \zeta\left(-a_{1}+z,-a_{2}+z\right)
$$

( $\mathrm{AET}$, Remark 2 therein), but this phenomenon does not survive in depth $k \geq 3$. Our table does not coincide with the table given in GZ, except at the diagonal arguments $(-a,-a)$ (as a consequence of the stuffle relations) and at points where the double zeta function is holomorphic in the two variables, i.e. $(-a,-b)$ with odd $a+b$.

It turns out that the method outlined above can be applied to more general situations: the Hurwitz multiple zeta functions:

$$
\zeta\left(s_{1}, \ldots, s_{k} ; v_{1}, \ldots, v_{k}\right):=\sum_{0<n_{k}<\cdots<n_{1}} \frac{1}{\left(n_{1}+v_{1}\right)^{s_{1}}} \cdots \frac{1}{\left(n_{k}+v_{k}\right)^{s_{k}}}, \quad v_{i} \geq 0,
$$

are renormalised exactly along the same lines, by considering symbols $\sigma_{s, v}(\xi):=$ $\chi(\xi)(|\xi|+v)^{-s}$ for some non negative real number $v$. Here again, at depth 2 we have

$$
\zeta\left(-a_{1},-a_{2} ; v\right)=\lim _{z \rightarrow 0} \zeta\left(-a_{1}+z,-a_{2}+z ; v\right) .
$$

Furthermore, the values of renormalised Hurwitz multiple zeta functions at nonpositive integer arguments:

$$
\zeta\left(-a_{1}, \ldots,-a_{k} ; v, \ldots, v\right)
$$

are rational numbers when $v \in \mathbb{Q}$, but the rationality issue for non-identical rational parameters $v_{1}, \ldots v_{k}$ is still an open question. Our proof for $v_{1}=\cdots=v_{k}=v$ relies on the algebra structure on the set of functions $\left\{\sigma_{-a, v}(x)=(x+v)^{a}, x>0, \quad a \in \mathbb{N}\right\}$. We show that our renormalized Hurwitz multiple zeta values at nonpositive integer arguments satisfy the two following identities:

$$
\zeta\left(-a_{1}, \ldots,-a_{k} ; v+1\right)=\zeta\left(-a_{1}, \ldots,-a_{k} ; v\right)-(v+1)^{a_{k}} \zeta\left(-a_{1}, \ldots,-a_{k-1} ; v+1\right)
$$

and

$$
\frac{d}{d v} \zeta\left(-a_{1}, \ldots,-a_{k} ; v\right)=\sum_{j=1}^{k} a_{j} \zeta\left(-a_{1}, \ldots,-a_{j-1},-a_{j}+1,-a_{j+1}, \ldots,-a_{k} ; v\right),
$$

which are well-known for arguments in the domain of convergence. Whereas the multiple zeta values $\zeta^{\text {alt }}\left(-a_{1},-a_{2} ; v\right):=\lim _{z \rightarrow 0} \zeta\left(-a_{1}+z,-a_{2}+z, \ldots,-a_{k}+z ; v\right)$ considered

${ }^{5}$ The case $k=1$ gives the Hurwitz zeta function with parameter $q=v+1$ since $\zeta(s ; v)=\zeta(s, v+1)$. This non-standard convention somewhat simplifies the notations when dealing with Hurwitz and ordinary multiple zeta functions simultaneously. 
in AET for non positive integers $a_{i}$ do not satisfy stuffle relations, they do satisfy the relations (9) and (10) as a result of the corrseponding identities of meromorphic functions (with respect to the variables $s_{j}$ ):

$$
\zeta\left(s_{1}, \ldots, s_{k} ; v+1\right)=\zeta\left(s_{1}, \ldots, s_{k} ; v\right)-(v+1)^{-s_{k}} \zeta\left(s_{1}, \ldots, s_{k-1} ; v+1\right)
$$

and:

$$
\frac{d}{d v} \zeta\left(s_{1}, \ldots, s_{k} ; v\right)=-\sum_{j=1}^{k} s_{j} \zeta\left(s_{1}, \ldots, s_{j-1}, s_{j}+1, s_{j+1}, \ldots, s_{k} ; v\right) .
$$

In the last part of the paper, we extend the renormalisation procedure described above to higher-dimensional iterated sums on $\mathbb{R}^{n}$ of the following type:

$$
\sum_{1 \leq\left|n_{k}\right|<\cdots<\left|n_{1}\right|} \sigma_{1} \otimes \cdots \otimes \sigma_{k} .
$$

Here |.| stands for the supremum norm on $\mathbb{R}^{n}$, and the $\sigma_{j}$ 's are radial functions $f_{j}$ ㅇ․| where $f_{j}$ is an ordinary symbol on $\mathbb{R}$ with support in $] 0,+\infty[$. Such a nested sum can be explicitly expressed as an ordinary (one-dimensional) iterated sum, namely:

$$
\sum_{1 \leq\left|n_{k}\right|<\cdots<\left|n_{1}\right|} \sigma_{1} \otimes \cdots \otimes \sigma_{k}=\sum_{1 \leq n_{k}<\cdots<n_{1}} A_{n} f_{1} \otimes \cdots \otimes A_{n} f_{k},
$$

where $A_{n}(t)=(2 t+1)^{n}-(2 t-1)^{n}$ is the natural interpolation of the number of points in the intersection of the sphere of radius $t$ with $\mathbb{Z}^{n}$.

This yields a natural way to define higher-dimensional renormalized Hurwitz multiple zeta values $\zeta_{n}\left(s_{1}, \ldots, s_{k} ; v_{1}, \ldots, v_{k}\right)$ in terms of renormalized nested sums of one-dimensional symbols. When $v_{1}=\cdots=v_{k}=v$, these turn out to be linear combinations with coefficients given by polynomial expressions in $v$ of renormalized onedimensional Hurwitz multiple zeta values, which converge for $\operatorname{Re}\left(s_{1}+\cdots+s_{m}\right)>n m$ for any $m \in\{1, \ldots, k\}$. An interesting but probably difficult problem is how to build similar renormalised higher dimensional multiple zeta values with the supremum norm replaced by the Euclidean norm, the main difficulty arising from the lack of an exact formula for the number of integer points in the euclidean sphere of given radius, centered at zero.

\section{Contents}

1 NESTED INTEGRALS OF SYMBOLS 8

1.1 Cut-off integrals of log-polyhomogeneous symbols . . . . . . . . . 8

1.1.1 Log-polyhomogeneous symbols . . . . . . . . . . . . . . 8

1.1.2 Cut-off integrals revisited . . . . . . . . . . . . . . . 9

1.1.3 Holomorphic families . . . . . . . . . . . . . . . . . . . . 10

1.2 Nested integrals on classical symbols . . . . . . . . . . . . . 11

1.3 Cut-off nested integrals of symbols . . . . . . . . . . . . . 13

1.4 A continuous analog of multiple zeta functions . . . . . . . . . . 14

1.5 Nested integrals of holomorphic families of symbols . . . . . . . . . 15

1.6 Shuffle relations for renormalised nested integrals of symbols . . . . . 17 
2 DISCRETE SUMS OF SYMBOLS 21

2.1 Discrete sums versus integrals of symbols . . . . . . . . . . . 21

2.2 The Riemann and Hurwitz zeta functions as regularised sums of a symbol 23

3 NESTED SUMS OF SYMBOLS

3.1 The Hoffman isomorphism and stuffle relations for discrete nested sums 26

3.2 Cut-off nested sums of symbols and multiple (Hurwitz) zeta functions 28

3.3 Renormalised nested sums of symbols . . . . . . . . . . . 31

4 RENORMALISED MULTIPLE ZETA FUNCTIONS 36

4.1 Stuffle relations for renormalised multiple zeta functions . . . . . . . . 36

4.2 Rationality of (Hurwitz) multiple zeta values at nonpositive arguments 37

4.3 An alternative renormalization method . . . . . . . . . . . . . . 44

4.4 Two identities preserved at nonpositive arguments . . . . . . . . 4 45

5 A HIGHER DIMENSIONAL ANALOG OF MULTIPLE ZETA FUNCTIONS

5.1 An algebra of radial symbols . . . . . . . . . . . . . . . . . 48

5.2 Higher-dimensional renormalised multiple zeta values . . . . . . . . . . 49

5.3 Explicit formulae for higher-dimensional multiple zeta values . . . . . 50

5.4 Higher-dimensional Hurwitz multiple zeta values renormalized at non positive integers ...................... 51 


\section{NESTED INTEGRALS OF SYMBOLS}

Throughout this part as well as parts 2 and $3,|\cdot|$ stands for a continuous norm on $\mathbb{R}^{n}$, smooth outside the origin.

\subsection{Cut-off integrals of log-polyhomogeneous symbols}

\subsubsection{Log-polyhomogeneous symbols}

We partly follow $\mathrm{L}$, Sections 2 and 3. For any complex number $\alpha$ and any nonnegative integer $k$ let us denote by $\mathcal{S}^{\alpha, k}\left(\mathbb{R}^{n}\right)$ the set of complex valued smooth functions on $\mathbb{R}^{n}$ which can be written $f(\xi)=\sum_{l=0}^{k} f_{l}(\xi) \log ^{l}|\xi|$ where the $f_{l}$ 's have the following asymptotic behaviour as $|\xi| \rightarrow \infty$ :

$$
f_{l}(\xi) \sim \sum_{j=0}^{\infty} f_{\alpha-j, l}(\xi)
$$

Here $f_{\alpha-j, l}(\xi)$ is positively homogeneous of degree $\alpha-j$. The notation $\sim$ stands for the existence, for any positive integer $N$ and any multi-index $\gamma$, of a positive constant $C_{\gamma, N}$ such that

$$
\left|\partial^{\gamma}\left(f_{l}(\xi)-\sum_{j=0}^{N} f_{\alpha-j, l}(\xi)\right)\right| \leq C_{\gamma, N}(1+|\xi|)^{\operatorname{Re} \alpha-N-1-|\gamma|}
$$

with $\partial^{\gamma}=\left(\frac{\partial}{\partial \xi_{1}}\right)^{\gamma_{1}} \cdots\left(\frac{\partial}{\partial \xi_{k}}\right)^{\gamma_{k}}$, with $|\gamma|=\gamma_{1}+\cdots+\gamma_{k}$ and where Re $\alpha$ stands for the real part of $\alpha$. We simplify notations setting $\mathcal{S}^{\alpha, k}:=\mathcal{S}^{\alpha, k}\left(\mathbb{R}^{n}\right)$ when there is no ambiguity on the dimension, which is one in most of the paper, except for Section 5 where it is $n>1$. We set:

$$
\mathcal{S}^{\alpha, *}:=\bigcup_{k=0}^{\infty} \mathcal{S}^{\alpha, k}
$$

and $\mathcal{S}^{*, k}$ stands for the linear span over $\mathbb{C}$ of all $\mathcal{S}^{\alpha, k}$ for $\alpha \in \mathbb{C}$. We also define:

$$
\mathcal{S}^{*, *}:=\bigcup_{k=0}^{\infty} \mathcal{S}^{*, k}
$$

which is a filtered algebra (with respect to the logarithmic power $k$ ) for the ordinary product of functions. If $f \in \mathcal{S}^{\alpha, k}$ and if there is an $l \in \mathbb{N}$ such that $f_{\alpha, l} \neq 0$ in (13) we call $\alpha$ the order of $f$. Note that the union $\bigcup_{\alpha \in-\mathbb{N}} \bigcup_{k=0}^{\infty} \mathcal{S}^{\alpha, k}$ is a subalgebra of $\mathcal{S}^{*, *}$, and that $\bigcup_{\alpha \in-\mathbb{N}} \mathcal{S}^{\alpha, 0}$ is a subalgebra of $\mathcal{S}^{*, 0}$. The real order of $f \in \mathcal{S}^{*, *}$ will be defined as:

$$
o(f)=\inf \left\{\lambda \in \mathbb{R}, \lim _{|\xi| \rightarrow+\infty}|\xi|^{-\lambda} f(\xi)=0\right\}
$$

hence $o(f)=\operatorname{Re} \alpha$ for $f \in \mathcal{S}^{\alpha, *}$ of order $\alpha$.

The algebra $\mathcal{S}^{*, *}$ (resp. the subalgebra $\mathcal{S}^{*, 0}$ ) coincides with the algebra of $\log$ polyhomogeneous (resp. classical) constant coefficient symbols on $\mathbb{R}^{n}$, which is often denoted by $C S^{*, *}$ (resp. $C S$ ) in the pseudodifferential literature. Typical examples of classical symbols are the polynomials in $n$ variables, and more generally complex 
powers of polynomials, which can be defined for any polynomial provided it nowhere vanishes on $\mathbb{R}^{n}$ and takes values outside an angular sector pointed at the origin of the complex plane. Log-polyhomogeneous symbols naturally appear by taking iterated primitives of suitable classical ones ([L], Section 3).

We recall results which belong to folklore knowledge, namely that the definition of $\mathcal{S}^{*, *}$ is independent of the choice of the norm. This is due to the fact that all norms are equivalent on a finite dimensional space: Let $|\cdot|$ and $|\cdot|_{1}$ be two norms smooth outside the origin. Let $f \in \mathcal{S}^{\alpha, k}$ which we write

$$
f(\xi)=\sum_{l=0}^{k} \sum_{j=0}^{K_{N}} f_{\alpha-j, l}(\xi) \log ^{l}|\xi|+f_{(N)}(\xi)
$$

with $f_{\alpha-j, l}$ positively homogeneous of order $\alpha-j$ and $f_{(N)}$ of order $\leq-N$. Then

$$
\begin{aligned}
f(\xi) & =\sum_{l=0}^{k} \sum_{j=0}^{K_{N}} f_{\alpha-j, l}(\xi) \log ^{l}|\xi|+f_{(N)}(\xi) \\
& =\sum_{l=0}^{k} \sum_{j=0}^{K_{N}} f_{\alpha-j, l}(\xi)\left(\log |\xi|_{1}+\log \frac{|\xi|}{|\xi|_{1}}\right)^{l}+f_{(N)}(\xi) \\
& =\sum_{l=0}^{k} \sum_{j=0}^{K_{N}} f_{\alpha-j, l}^{1}(\xi) \log ^{l}|\xi|_{1}+f_{(N)}(\xi)
\end{aligned}
$$

where we have set

$$
f_{\alpha-j, i}^{1}(\xi):=\sum_{l=0}^{k} f_{\alpha-j, l}(\xi)\left(\begin{array}{l}
l \\
i
\end{array}\right) \log ^{l-i} \frac{|\xi|}{|\xi|_{1}}
$$

It follows immediately that $f_{\alpha-j, i}^{1}$ is positively homogeneous of order $\alpha-j$, which proves the claim.

\subsubsection{Cut-off integrals revisited}

Cut-off integrals can be defined on the class $\mathcal{S}^{*, *}$ of (constant coefficient) log-polyhomogeneous symbols $[\mathrm{L}$. The result depends on the choice of the norm. We recall however the asymptotic behaviour of integrals on balls, which can be derived independently of the specific norm chosen.

Proposition 1 Let $d_{S} \xi$ be the volume measure on the unit sphere induced by the canonical Lebesgue measure on $\mathbb{R}^{n}$. Let $B(0, R)$ be the closed ball in $\mathbb{R}^{n}$ centered at 0 with radius $R$ and for any $0 \leq r \leq R$, let us set $B(r, R)=B(0, R)-B(0, r)$. Then for any fixed $r$ and for any $f \in \mathcal{S}^{*, k}$ displayed as in (14), the integral $\int_{B(r, R)} f(\xi) d \xi$ has an asymptotic expansion as $R \rightarrow+\infty$ of the type

$$
\begin{gathered}
\int_{B(r, R)} f(\xi) d \xi \quad \sim_{R \rightarrow \infty} \quad C_{r}(f)+\sum_{j=0, \alpha-j+n \neq 0}^{\infty} \sum_{l=0}^{k} P_{l}\left(f_{\alpha-j, l}\right)(\log R) R^{\operatorname{Re} \alpha-j+n} \\
+\quad \sum_{l=0}^{k} r_{l}(f) \log ^{l+1} R
\end{gathered}
$$


where the $r_{l}(f)$ are positive constants depending on $f_{l,-n}, P_{l}\left(f_{\alpha-j, l}\right)(X)$ is a polynomial of degree $l$ with coefficients depending on $f_{\alpha-j, l}$ and where the constant term $C_{r}(f)$ corresponds to the finite part:

$$
\begin{aligned}
C_{r}(f) & :=\int_{\mathbb{R}^{n}} f_{(N)}(\xi) d \xi+\int_{B(r, 1)} f(\xi) d \xi \\
& +\sum_{j=0, \alpha-j+n \neq 0}^{N} \sum_{l=0}^{k} \frac{(-1)^{l+1} l !}{(\alpha-j+n)^{l+1}} \int_{|\xi|=1} f_{\alpha-j, l}(\xi) d_{S} \xi
\end{aligned}
$$

which is independent of $N$ for $N \geq \alpha+n-1$.

Proof: A proof can be found e.g. in $\mathrm{L}$ (see also $\mathrm{MP}$ ) and references therein for the case $r=0$. The case $r>0$ then easily follows subtracting $\int_{B(0, r)} f(\xi) d \xi$ from the constant term so that $C_{r}(f)=C_{0}(f)-\int_{B(0, r)} f(\xi) d \xi$.

The cut-off integral of $f$ outside the ball of radius $r$ is defined as the finite part $C_{r}(f)$, and is denoted by:

$$
f_{r \leq|\xi|} f(\xi) d \xi
$$

It coincides with the ordinary integral when the latter converges, but may depend on the choice of the norm in the general case.

\subsubsection{Holomorphic families}

Let us first recall what we mean by a holomorphic family of elements of some topological vector space $\mathcal{A}$. Let $W \subset \mathbb{C}$ be a complex domain. A family $\{f(z)\}_{z \in W} \subset \mathcal{A}$ is holomorphic at $z_{0} \in W$ if the corresponding function $f: W \rightarrow \mathcal{A}$ admits a Taylor expansion in a neighbourhood $N_{z_{0}}$ of $z_{0}$

$$
f(z)=\sum_{k=0}^{\infty} f^{(k)}\left(z_{0}\right) \frac{\left(z-z_{0}\right)^{k}}{k !}
$$

which is convergent, uniformly on compact subsets of $N_{z_{0}}$, with respect to the topology on $\mathcal{A}$. The vector spaces of functions we consider here are $C\left(\mathbb{R}^{n}, \mathbb{C}\right)$ and $C^{\infty}\left(\mathbb{R}^{n}, \mathbb{C}\right)$ equipped with their usual topologies, namely uniform convergence on compact subsets, and uniform convergence of all derivatives on compact subsets respectively.

Definition 1 Let $k$ be a non-negative integer, and let $W$ be a domain in $\mathbb{C}$. A simple holomorphic family of log-polyhomogeneous symbols $f(z) \in \mathcal{S}^{*, k}$ parametrised by $W$ of order $\alpha: W \rightarrow \mathbb{C}$ means a holomorphic family $f(z)(\xi):=f(z, \xi)$ of smooth functions on $\mathbb{R}^{n}$ for which:

1. $f(z)(\xi)=\sum_{l=0}^{k} f_{l}(\xi) \log ^{l}|\xi|$ with

$$
f_{l}(z)(\xi) \sim \sum_{j \geq 0} f(z)_{\alpha(z)-j, l}(\xi) .
$$

Here $\alpha: W \rightarrow \mathbb{C}$ is a holomorphic map and $f(z)_{\alpha(z)-j, l}$ is positively homogeneous of degree $\alpha(z)-j$. 
2. For any positive integer $N$ there is some positive integer $K_{N}$ such that the remainder term

$$
f_{(N)}(z)(\xi):=f(z)(\xi)-\sum_{l=0}^{k} \sum_{j=0}^{K_{N}} f(z)_{\alpha(z)-j, l}(\xi) \log ^{l}|\xi|=o\left(|\xi|^{-N}\right)
$$

is holomorphic in $z \in W$ as a function of $\xi$ and verifies for any $\epsilon>0$ the following estimates:

$$
\partial_{\xi}^{\beta} \partial_{z}^{k} f_{(N)}(z)(\xi)=o\left(|\xi|^{-N-|\beta|+\epsilon}\right)
$$

for $k \in \mathbb{N}$ and $\beta \in \mathbb{N}^{n}$.

A holomorphic family of log-polyhomogeneous symbols is a finite linear combination (over $\mathbb{C}$ ) of simple holomorphic families.

Note that the notion of holomorphic order $\alpha(z)$ only makes sense for simple holomorphic families. Cut-off integrals of log-polyhomogeneous simple holomorphic families behave as follows: $[\mathrm{L}$ :

Proposition 2 Given a non-negative integer $k$, let $\{f(z)\}$ be a family in $\mathcal{S}^{*, k}$ holomorphic on $W \subset \mathbb{C}$ and simple. Let us assume that the order $\alpha(z)$ of $f(z)$ is of the form $\alpha(z)=-q z+\alpha(0)$ with $q \neq 0$. Then the map

$$
z \mapsto f_{\mathbb{R}^{n}} f(z)(\xi)
$$

is meromorphic with poles of order $\leq k+1$ in the discrete set:

$$
\alpha^{-1}\left(\left[-n,+\infty[\cap \mathbb{Z})=\left\{\frac{\alpha(0)+n-j}{q}, j \in \mathbb{N}\right\} .\right.\right.
$$

\subsection{Nested integrals on classical symbols}

Let us introduce the following nested integrals of symbols on compact sets:

Definition 2 Given $R>r>0$,

$$
\begin{aligned}
& \int_{B(r, R)}^{\text {Chen }} \sigma_{1} \otimes \cdots \otimes \sigma_{k} \\
:= & \int_{r \leq\left|\xi_{k}\right| \leq\left|\xi_{k-1}\right| \leq \cdots \leq\left|\xi_{1}\right| \leq R} d \xi_{1} \cdots d \xi_{k} \sigma_{1}\left(\xi_{1}\right) \sigma_{2}\left(\xi_{2}\right) \cdots \sigma_{k}\left(\xi_{k}\right) \\
= & \int_{r \leq r_{k} \leq r_{k-1} \leq \cdots \leq r_{1} \leq R} d r_{1} \cdots d r_{k} f_{1}\left(r_{1}\right) \cdots f_{k}\left(r_{k}\right)
\end{aligned}
$$

where we have set $f_{i}(r):=r^{n-1} \int_{|\xi|=1} \sigma_{i}(r \xi) d_{S} \xi$.

These nested integrals correspond to ordinary nested (or iterated) integrals

$$
\int_{r \leq r_{k} \leq \cdots \leq r_{1} \leq R} \omega_{1} \wedge \cdots \wedge \omega_{k}
$$

with $\omega_{i}(t)=f_{i}(t) d t$. As such they enjoy the usual properties of one-dimensional nested integrals (see e.g [Ch, or Appendix XIX.11 in $\mathrm{Ka}]$ ): 
Definition 3 The tensor algebra $\mathcal{T}\left(\mathcal{S}^{*, *}\right):=\bigoplus_{k=0}^{\infty}\left(\mathcal{S}^{*, *}\right)^{\otimes k}$ (over the base field $\mathbb{C}$ ) is equipped with a shuffle product:

$$
\left(\sigma_{1} \otimes \cdots \otimes \sigma_{k}\right) \amalg\left(\sigma_{k+1} \otimes \cdots \otimes \sigma_{k+l}\right):=\sum_{\tau \in \Sigma_{k ; l}} \sigma_{\tau^{-1}(1)} \otimes \cdots \otimes \sigma_{\tau^{-1}(k+l)}
$$

where $\tau$ runs over the set $\Sigma_{k ; l}$ of $(k, l)$-shuffles, i.e. permutations $\tau$ on $\{1, \ldots, k+l\}$ satisfying $\tau(1)<\cdots<\tau(k)$ and $\tau(k+1)<\cdots<\tau(k+l)$.

The shuffle product and the deconcatenation coproduct:

$$
\Delta\left(\sigma_{1} \otimes \cdots \otimes \sigma_{k}\right):=\sum_{j=0}^{k}\left(\sigma_{1} \otimes \cdots \otimes \sigma_{j}\right) \bigotimes\left(\sigma_{j+1} \otimes \cdots \otimes \sigma_{k}\right)
$$

endow $\mathcal{T}\left(\mathcal{S}^{*, *}\right)$ with a structure of connected graded commutative Hopf algebra. This algebraic construction holds if we replace $\mathcal{S}^{*, *}$ by any vector space $\mathrm{H} 2$. As in the case of ordinary nested integrals, we have the following shuffle relations:

Proposition 3 For any $\sigma, \tau \in \mathcal{T}\left(\mathcal{S}^{*, *}\right)$

$$
\int_{B(r, R)}^{\text {Chen }} \sigma \amalg \tau=\int_{B(r, R)}^{\text {Chen }} \sigma \int_{B(r, R)}^{\text {Chen }} \tau \text {. }
$$

Proof: We may assume that $\sigma$ and $\tau$ are indecomposable elements. Setting $\sigma=$ $\sigma_{1} \otimes \cdots \otimes \sigma_{k}$ and $\tau=\tau_{1} \otimes \cdots \otimes \tau_{l}, f_{i}(r)=r^{n-1} \int_{|\xi|=1} \sigma_{i}(r \xi) d_{S} \xi$ and $g_{j}(r)=$ $r^{n-1} \int_{|\xi|=1} \tau_{j}(r \xi) d_{S} \xi$ we have:

$$
\begin{aligned}
\int_{B(r, R)}^{\text {Chen } \sigma ш \tau} & =\int_{[r, R]}^{\text {Chen }}\left(f_{1} \otimes \cdots \otimes f_{k}\right) \amalg\left(g_{1} \otimes \cdots \otimes g_{l}\right) \\
& =\left(\int_{[r, R]}^{\text {Chen }} f_{1} \otimes \cdots \otimes f_{k}\right)\left(\int_{[r, R]}^{\text {Chen }} g_{1} \otimes \cdots \otimes g_{l}\right) \\
& =\int_{B(r, R)}^{\text {Chen }} \sigma \int_{B(r, R)}^{\text {Chen }} \tau .
\end{aligned}
$$

where we have used the shuffle property for usual nested Chen integrals between the bounds $r$ and $R$.

Finally, let us recall Chen's lemma:

Proposition 4 Given $\sigma=\otimes_{i=1}^{k} \sigma_{i} \in \otimes_{i=1}^{k} \mathcal{S}^{*, *}$ we have:

$$
\begin{aligned}
\int_{B(r, R)}^{\text {Chen }} \sigma & =\int_{B(r, \Lambda)}^{\text {Chen }} \sigma+\int_{B(\Lambda, R)}^{\text {Chen }} \sigma \\
& +\sum_{j=1, \ldots, k-1} \int_{B(r, \Lambda)}^{\text {Chen } \sigma_{i_{1}} \otimes \cdots \otimes \sigma_{i_{j}} \int_{B(\Lambda, R)}^{\text {Chen }} \sigma_{i_{j+1}} \otimes \cdots \otimes \sigma_{i_{k}} .}
\end{aligned}
$$

To summarise these two facts, the three linear maps $\int_{B(r, \Lambda)}^{\text {Chen }}, \int_{B(\Lambda, R)}^{\text {Chen }}$ and $\int_{B(r, R)}^{\text {Chen }}$ are characters of the Hopf algebra $\mathcal{T}\left(\mathcal{S}^{*, *}\right)$, and moreover satisfy the relation:

$$
\int_{B(r, R)}^{\text {Chen }}=\int_{B(r, \Lambda)}^{\text {Chen }} \star \int_{B(\Lambda, R)}^{\text {Chen }},
$$

where $\star$ stands for the convolution product. 


\subsection{Cut-off nested integrals of symbols}

In this paragraph we let $R$ tend to infinity, which requires a regularisation since the integrals do not a priori converge when $R \rightarrow \infty$. Let us first recall how nested integrations can turn classical symbols into log-polyhomogeneous symbols.

Lemma $1[M P]$ Let $r \geq 0$. The following operator on $C^{\infty}\left(\mathbb{R}^{n}\right)$ :

$$
\tilde{P}_{r}(f)(\eta):=\int_{r \leq|\xi| \leq|\eta|} f(\xi) d \xi
$$

maps $\mathcal{S}^{*, k-1}$ to $\mathcal{S}^{*, k}$ for any positive integer $k$.

Proof: It follows from setting $R=|\eta|$ in equation (15). The smoothness of the norm outside the origin ensures that whenever $f$ is a symbol, then the resulting map $\tilde{P}_{r}(f)$ is also a symbol.

As a result of Proposition 3 the map $\tilde{P}_{r}$ satisfies the following Rota-Baxter relation (see MP] Theorem 3):

$$
\tilde{P}_{r}(f) \tilde{P}_{r}(g)=\tilde{P}_{r}\left(f \tilde{P}_{r}(g)\right)+\tilde{P}_{r}\left(\tilde{P}_{r}(f) g\right) .
$$

Iterating Lemma 1 we get:

Lemma 2 Given $\sigma_{i} \in \mathcal{S}^{\alpha_{i}, 0}$ and $\sigma:=\otimes_{i=1}^{k} \sigma_{i}$, for any $r \geq 0$ and $k \geq 2$ the function

$$
\sigma_{r}^{\text {Chen }}: \xi \mapsto \sigma_{1}(\xi) \int_{B(r,|\xi|)}^{\text {Chen }} \sigma_{2} \otimes \cdots \otimes \sigma_{k}
$$

lies in $\mathcal{S}^{*, k-1}$ as a linear combination of log-polyhomogeneous functions of order $\alpha_{1}+$ $\alpha_{2}+\cdots+\alpha_{j}+(j-1) n, j=1, \ldots, k$. It has real order $\omega_{1}$, where we successively define $\omega_{k}=\operatorname{Re} \alpha_{k}$ and $\omega_{j}=\operatorname{Re} \alpha_{j}+\max \left(0, \omega_{j+1}+n\right)$.

We can define the cut-off nested integral of $\sigma$ as the cut-off integral of the symbol $\sigma_{r}^{\text {Chen }}$, namely:

Definition 4 Given $\sigma_{i} \in \mathcal{S}^{\alpha_{i}, 0}, i=1, \ldots, k$, setting $\sigma=\otimes_{i=1}^{k} \sigma_{i}$, for any $r>0$ we set

$$
f_{r}^{\text {Chen }} \sigma:=\mathrm{fp}_{R \rightarrow \infty} \int_{B(r, R)}^{\text {Chen }} \sigma .
$$

Remark 1 It follows from the above lemma that if $\sum_{j=1}^{m} \operatorname{Re} \alpha_{j}<-n m$ for any $m \in\{1, \ldots, k\}$, then the symbol $\sigma_{r}^{\text {Chen }}$ also has real order $<-n$ so that the nested integral converges and the cut-off integrals become ordinary integrals.

We now want to extend the shuffle relation (19) in the $R \rightarrow \infty$ limit.

Theorem 1 Given $\sigma \in \bigotimes_{i=1}^{k} \mathcal{S}^{\alpha_{i}, 0}$ and $\tau \in \bigotimes_{j=1}^{l} \mathcal{S}^{\beta_{j}, 0}$ then,

1. if all the partial sums $\alpha_{1}+\alpha_{2}+\cdots+\alpha_{m}+\beta_{1}+\beta_{2}+\cdots+\beta_{p}$ with $m \in\{1, \ldots, k\}$, $p \in\{1, \ldots, l\}$ are non-integer valued, the following shuffle relation holds:

$$
f_{r}^{\text {Chen }} \sigma ш \tau=\left(f_{r}^{\text {Chen }} \sigma\right)\left(f_{r}^{\text {Chen }} \tau\right) \text {. }
$$


2. If one of the two conditions: $\operatorname{Re}\left(\alpha_{1}+\cdots+\alpha_{m}\right)<-n m$ for any $m \in\{1, \ldots, k\}$, or $\operatorname{Re}\left(\beta_{1}+\cdots+\beta_{m}\right)<-n m$ for any $m \in\{1, \ldots, l\}$ holds, the shuffle relation also holds.

3. If $\operatorname{Re}\left(\alpha_{1}+\cdots+\alpha_{m}\right)<-n m$ for any $m \in\{1, \ldots, k\}$ and $\operatorname{Re}\left(\beta_{1}+\cdots+\beta_{m}\right)<$ $-n m$ for any $m \in\{1, \ldots, l\}$ both hold, the shuffle relation moreover becomes an equality of ordinary nested integrals.

Proof: This follows from taking finite parts when $R \rightarrow \infty$ in Proposition 3

1. Let us now assume that all the partial sums $\alpha_{1}+\alpha_{2}+\cdots+\alpha_{m}+\beta_{1}+\beta_{2}+\cdots+\beta_{p}$ with $m \in 1, \ldots, k, p \in\{1, \ldots, l\}$ are non-integer valued. Under this assumption, the finite part of the product of the integrals coincides with the product of the finite parts of the integrals. Let us make this statement more precise: On the right hand side of

$$
\int_{B(r, R)}^{\text {Chen }} \sigma \amalg \tau=\left(\int_{B(r, R)}^{\text {Chen }} \sigma\right)\left(\int_{B(r, R)}^{\text {Chen }} \tau\right),
$$

one might expect cancellations of divergences when $R \rightarrow \infty$ which could lead to new contributions to the finite part. Since the asymptotic expansion of

$$
\int_{B(r, R)}^{\text {Chen }} \sigma=\int_{r \leq|\xi| \leq R} \sigma_{r}^{\text {Chen }}(\xi) d \xi
$$

when $R \rightarrow \infty$ involves powers $R^{\alpha_{1}+\alpha_{2}+\cdots+\alpha_{m}+m n-j}$ for non-negative integers $j$ combined with powers of $\log R$ (and similarly for $\int_{B(r, R)}^{\text {Chen }} \tau$ ), such cancellations of divergences do not occur if $\alpha_{1}+\alpha_{2}+\cdots+\alpha_{m}+\beta_{1}+\beta_{2}+\cdots+\beta_{p}$ do not lie in $[-n, \infty[\cap \mathbb{Z}$.

2. If one of the conditions of item 2 holds, then one of the expressions $\int_{B(r, R)}^{\text {Chen }} \sigma$ or $\int_{B(r, R)}^{C h e n} \tau$ converges in the limit $R \rightarrow \infty$ so that such cancellations do not occur either. As a result, the shuffle property also holds in the limit.

3. If both conditions of item 2 hold, all the integrals converge and the finite parts are ordinary limits. Taking the limits when $R \rightarrow \infty$ in Proposition 3 yields the result.

\subsection{A continuous analog of multiple zeta functions}

We define a continuous analog of multiple zeta functions via nested integrals of symbols. For $\epsilon>0$, let $\chi_{\epsilon}$ be a smooth cut-off function on $\mathbb{R}^{n}$ that vanishes in the ball $B\left(0, \frac{\epsilon}{4}\right)$ and is constant equal to 1 outside the ball $B\left(0, \frac{\epsilon}{2}\right)$. The symbols

$$
\sigma_{i}(\xi)=\chi_{\epsilon}(\xi)|\xi|^{-s_{i}}
$$

lie in $\mathcal{S}^{*, 0}$. For $\underline{s}:=\left(s_{1}, \ldots, s_{k}\right) \in \mathbb{C}^{k}$ we set $\sigma_{\underline{s}}:=\sigma_{s_{1}} \otimes \cdots \otimes \sigma_{s_{k}}$ and define for $r \geq \frac{\epsilon}{2}$,

$$
\begin{aligned}
\tilde{\zeta}_{n}^{r}(\underline{s}) & :=f_{r \leq\left|\xi_{k}\right| \leq \cdots \leq\left|\xi_{1}\right|} \sigma_{\underline{s}} \\
& =f_{r \leq\left|\xi_{k}\right| \leq \cdots \leq\left|\xi_{1}\right|} d \xi_{1} \cdots d \xi_{k} \prod_{i=1}^{k}\left|\xi_{i}\right|^{-s_{i}} .
\end{aligned}
$$


If $s_{1}>n$ and $s_{1}+\cdots+s_{k}>n k$ then, by Remark $\mathbb{1} \tilde{\zeta}_{n}^{r}(\underline{s})$ converges and the regularised integrals reduce to ordinary ones. In dimension one we write:

$$
\tilde{\zeta}^{r}(\underline{s}):=f_{r \leq t_{k} \leq t_{k-1} \leq \cdots \leq t_{1}} d t_{1} \cdots d t_{k} \prod_{i=1}^{k} t_{i}^{-s_{i}} .
$$

This corresponds to $2^{-k} \tilde{\zeta}_{1}^{r}(\underline{s})$ the norm chosen being the usual absolute value. This clearly does not extend to $r=0$.

Remark 2 Note the analogy with the usual multiple zeta functions, an analogy which was made precise in [MP] via an Euler-MacLaurin formula.

The following proposition gives an analog of the "second shuffle relations" ENR, also called stuffle relations for multiple zeta functions (but without "diagonal terms", which arise only in the discrete setting. As such they appear as "ordinary" shuffle relations).

Proposition 5 Let $\underline{s}=\left(s_{1}, \ldots, s_{k}\right) \in \mathbb{R}^{k}, \underline{s}^{\prime}=\left(s_{k+1}, \ldots, s_{k+l}\right) \in \mathbb{R}^{l}$ such that $s_{1}+$ $\cdots+s_{m}>n m$ for any $m \in\{1, \ldots, k\}$ and $s_{k+1}+\cdots+s_{k+m}>n m$ for any $m \in$ $\{1, \ldots, k\}$. For any $r \geq 0$

$$
\tilde{\zeta}_{n}^{r}(\underline{s}) \tilde{\zeta}_{n}^{r}\left(\underline{s}^{\prime}\right)=\tilde{\zeta}_{n}^{r}\left(\underline{s} \amalg \underline{s}^{\prime}\right) .
$$

where $\underline{s} \amalg \underline{s}^{\prime}$ is defined as usual by:

$$
\left(s_{1}, \ldots, s_{k}\right) \amalg\left(s_{k+1}, \ldots, s_{k+l}\right)=\sum_{\tau \in \Sigma_{k ; l}}\left(s_{\tau^{-1}(1)}, \ldots, s_{\tau^{-1}(k+l)}\right) .
$$

Proof: It follows from applying the first item of Theorem 1 to $\sigma=\sigma_{\underline{\underline{s}}}$ and $\tau=\sigma_{\underline{\underline{s}^{\prime}}}$ since

$$
\sigma_{\underline{\underline{s}}} \amalg \sigma_{\underline{\underline{s}}^{\prime}}=\sigma_{\underline{\underline{s}} \amalg \underline{s}^{\prime}} .
$$

When $n=1, \epsilon<r$ and $|\cdot|$ is the usual absolute value, this yields:

$$
\tilde{\zeta}^{r}(\underline{s}) \tilde{\zeta}^{r}\left(\underline{s}^{\prime}\right)=\tilde{\zeta}^{r}\left(\underline{s} \amalg \underline{s}^{\prime}\right) .
$$

We also describe a continuous analog of polylogarithms in the Appendix.

\subsection{Nested integrals of holomorphic families of symbols}

Let us briefly recall the notion of holomorphic regularisation inspired by $\mathrm{KV}$.

Definition 5 A holomorphic regularisation procedure on $\mathcal{S}^{*, *}$ is a map

$$
\begin{aligned}
\mathcal{R}: \mathcal{S}^{*, *} & \rightarrow \operatorname{Hol}_{W}\left(\mathcal{S}^{*, *}\right) \\
f & \mapsto\{f(z)\}_{z \in W}
\end{aligned}
$$

where $W$ is an open subset of $\mathbb{C}$ containing 0 , and $\operatorname{Hol}_{W}\left(\mathcal{S}^{*, *}\right)$ is the algebra of holomorphic families in $\mathcal{S}^{*, *}$ as defined in Section 1, such that for any $f \in \mathcal{S}^{*, *}$,

1. $f(0)=f$, 
2. the holomorphic family $f(z)$ can be written as a linear combination of simple ones:

$$
f(z)=\sum_{j=1}^{k} f_{j}(z)
$$

the holomorphic order $\alpha_{j}(z)$ of which verifying $\operatorname{Re} \alpha_{j}^{\prime}(z)<0$ for any $z \in W$ and any $j \in\{1, \ldots, k\}$.

The holomorphic regularisation $\mathcal{R}$ is simple if for any log-polyhomogeneous symbol $f \in \mathcal{S}^{\alpha, k}$ the holomorphic family $\mathcal{R}(f)$ is simple.

A similar definition holds with suitable subalgebras of $\mathcal{S}^{*, *}$, e.g. classical symbols $\mathcal{S}^{*, 0}$ instead of log-polyhomogeneous ones. Simple holomorphic regularisation procedures naturally arise in physics:

Example 1 Let $z \mapsto \tau(z) \in \mathcal{S}^{*, 0}$ be a holomorphic family of classical symbols such that $\tau(0)=1$ and $\tau(z)$ has holomorphic order $t(z)$ with $\operatorname{Re} t^{\prime}(z)<0$. Then

$$
\mathcal{R}: \sigma \mapsto \sigma(z):=\sigma \tau(z)
$$

yields a holomorphic regularisation on $\mathcal{S}^{*, *}$ as well as on $\mathcal{S}^{*, 0}$. Choosing $\tau(z)(\xi):=$ $\chi(\xi)+(1-\chi(\xi))\left(H(z)|\xi|^{-z}\right)$ where $H$ is a scalar valued holomorphic map such that $H(0)=1$, and where $\chi$ is a cut-off function as defined in the introduction, we get

$$
\mathcal{R}(\sigma)(z)(\xi)=\chi(\xi) \sigma(\xi)+(1-\chi(\xi))\left(H(z) \sigma|\xi|^{-z}\right) .
$$

Dimensional regularisation commonly used in physics is of this type, where $H$ is expressed in terms of Gamma functions which account for a "complexified" volume of the unit sphere. When $H \equiv 1$, such a regularisation $\mathcal{R}$ is called Riesz regularisation.

From a given simple holomorphic regularisation procedure $\mathcal{R}: \sigma \mapsto \sigma(z)$ on $\mathcal{S}^{*, 0}$ which yields ordinary regularised integrals

$$
f_{r}^{\mathcal{R}} \sigma:=\mathrm{fp}_{z=0} f_{r \leq|\xi|} \sigma(z)(\xi) d \xi, \quad \forall r \geq 0,
$$

we wish to build regularised nested integrals

$$
f_{r}^{\mathcal{R}, \text { Chen }} \sigma_{1} \otimes \cdots \otimes \sigma_{k}:=f_{r \leq\left|\xi_{k}\right| \leq \cdots \leq\left|\xi_{1}\right|}^{\mathcal{R}} \sigma_{1}\left(\xi_{1}\right) \cdots \sigma_{k}\left(\xi_{k}\right) d \xi_{1} \cdots d \xi_{k}
$$

with the following

1. For $k=1$, the nested integral coincides with the ordinary regularised integral $f_{r}^{\mathcal{R}}$.

2. It coincides with the ordinary Chen nested integral

$$
\int_{r}^{\text {Chen }} \sigma_{1} \otimes \cdots \otimes \sigma_{k}:=\int_{r \leq\left|\xi_{k}\right| \leq \cdots \leq\left|\xi_{1}\right|} \sigma_{1}\left(\xi_{1}\right) \cdots \sigma_{k}\left(\xi_{k}\right) d \xi_{1} \cdots d \xi_{k}
$$

on symbols $\sigma_{1}, \ldots, \sigma_{k}$ with respective orders $\alpha_{1}, \ldots, \alpha_{k}$ such that $\operatorname{Re}\left(\alpha_{1}+\cdots+\right.$ $\left.\alpha_{m}\right)<-n m$ for any $m \in\{1, \ldots, k\}$. 
Given a simple holomorphic regularisation $\mathcal{R}: \sigma \mapsto \sigma(z)$ on $\mathcal{S}^{*, 0}$, we can assign to $\sigma=\sigma_{1} \otimes \cdots \otimes \sigma_{k}$ holomorphic maps

$$
z \mapsto \tilde{\mathcal{R}}(\sigma)(z):=\sigma_{1}(z) \otimes \cdots \otimes \sigma_{k}(z) .
$$

Clearly, for any tensor products $\sigma=\sigma_{1} \otimes \cdots \otimes \sigma_{k}$ and $\tau=\tau_{1} \otimes \cdots \otimes \tau_{l}$ we have $\tilde{\mathcal{R}}(\sigma \otimes \tau)=\tilde{\mathcal{R}}(\sigma) \otimes \tilde{\mathcal{R}}(\tau)$, so that the regularisation is compatible with the shuffle product:

$$
\tilde{\mathcal{R}}(\sigma \amalg \tau)=\tilde{\mathcal{R}}(\sigma) \amalg \tilde{\mathcal{R}}(\tau) .
$$

Proposition 6 Let $\mathcal{R}: \sigma \mapsto\{\sigma(z)\}_{z \in W}$ be a holomorphic regularisation procedure defined on a neighbourhood $W$ of 0 in the complex plane. Given any non negative real number $r$, for any $\sigma_{i} \in \mathcal{S}^{\alpha_{i}, 0}, i=1, \ldots, k$, the map

$$
z \mapsto f_{r}^{\text {Chen }} \sigma_{1}(z) \otimes \cdots \otimes \sigma_{k}(z)
$$

is meromorphic with poles of order at most $k$. If moreover the holomorphic regularisation is simple and if for any $i \in\{1, \ldots, k\}$ the symbol $\sigma_{i}(z)$ has order $\alpha_{i}(z)=$ $-q z+\alpha_{i}(0)$ affine in $z$ with $q>0$, the poles lie in the discrete set of points $W \cap D_{k}$, with:

$$
D_{k}:=\bigcup_{j=1}^{k}\left\{\frac{\sum_{i=1}^{j} \alpha_{i}(0)+j n-\gamma}{j q}, \gamma \in \mathbb{N}\right\} .
$$

Proof: This result extends Theorem 5 in $\underline{\mathrm{MP}}$ in the case $r=0$. The first assertion follows from

$$
f_{r}^{\text {Chen }} \sigma(z)=f_{|\xi| \geq r}(\sigma(z))^{\text {Chen }}(\xi) d \xi .
$$

The second assertion comes from the fact that by Lemma 2. $(\sigma(z))^{\text {Chen }}$ lies in $\mathcal{S}^{*, k-1}$ as a linear combination of functions in $\mathcal{S}^{*, j-1}$ of order $\alpha_{1}(z)+\alpha_{2}(z)+\cdots+\alpha_{j}(z)+(j-1) n$, $j=1, \ldots, k$. Applying Proposition2 we infer that the map $z \mapsto f_{|\xi| \geq r}(\sigma(z))^{\text {Chen }}(\xi) d \xi$ has poles at $z_{j, \gamma}:=\frac{\sum_{i=1}^{j} \alpha_{i}(0)+j n-\gamma}{j q}, \gamma \in \mathbb{N}$. A closer look shows that the symbols of order $\alpha_{1}(z)+\cdots+\alpha_{j}(z)+(j-1) n$ arise from the $j$-th outermost integration so that poles at $z_{j, \gamma}$ are of order $j$.

\subsection{Shuffle relations for renormalised nested integrals of sym- bols}

Proposition 7 Given an open neighbourhood $W$ of 0 in $\mathbb{C}$ and a holomorphic regularisation $\mathcal{R}: \tau \mapsto\{\tau(z)\}_{z \in W}$ on $\mathcal{S}^{*, 0}$, for any non-negative real number $r$ the map

$$
\begin{aligned}
\Phi_{r}^{\mathcal{R}}: \mathcal{T}\left(\mathcal{S}^{*, 0}\right) & \rightarrow \mathcal{M}(W) \\
\sigma & \mapsto f_{r}^{\text {Chen }} \tilde{\mathcal{R}}(\sigma)
\end{aligned}
$$

defines a character from the commutative algebra $\left(\mathcal{T}\left(\mathcal{S}^{*, 0}\right)\right.$,ш $)$ to the algebra $\mathcal{M}(W)$ of meromorphic functions on $W$. 
Proof: By Proposition 6. $\Phi_{r}^{\mathcal{R}}$ is $\mathcal{M}(W)$-valued. Applying Theorem 1 to $\tilde{\mathcal{R}}(\sigma)(z)$ and $\tilde{\mathcal{R}}(\tau)(z)$ for any two $\sigma, \tau \in \mathcal{T}\left(\mathcal{S}^{*, 0}\right)$, which makes sense outside a discrete set of complex numbers $z$, we have by (30):

$$
\begin{aligned}
\Phi_{r}^{\mathcal{R}}(\sigma \amalg \tau) & =f_{r}^{\text {Chen }}(\tilde{\mathcal{R}}(\sigma \amalg \tau)(z)) \\
& =f_{r}^{\text {Chen }}(\tilde{\mathcal{R}}(\sigma)(z) \amalg \tilde{\mathcal{R}}(\tau)(z)) \\
& =\left(f_{r}^{\text {Chen }} \tilde{\mathcal{R}}(\sigma)(z)\right)\left(f_{r}^{\text {Chen }} \tilde{\mathcal{R}}(\tau)(z)\right) \\
& =\Phi_{r}^{\mathcal{R}}(\sigma) \Phi_{r}^{\mathcal{R}}(\tau)
\end{aligned}
$$

as an equality of meromorphic functions, from which the above proposition follows.

Theorem 2 Let $W \subset \mathbb{C}$ be an open neighbourhood of 0 . Given a holomorphic regularisation $\mathcal{R}: \sigma \mapsto\{\sigma(z)\}_{z \in W}$ on classical symbols, for any non-negative real number $r$ the regularised integral $f_{r}^{\mathcal{R}}$ extends to a character

$$
\phi_{r}^{\mathcal{R}}:\left(\mathcal{T}\left(\mathcal{S}^{*, 0}\right), \amalg\right) \rightarrow \mathbb{C}
$$

on the tensor algebra equipped with the shuffle product, given by $\phi_{r}^{\mathcal{R}}:=\Phi_{r,+}^{\mathcal{R}}(0)$ from the Birkhoff decomposition of the $\mathcal{M}(W)$-valued character $\Phi_{r}^{\mathcal{R}}$. It coincides with $f_{r}^{\text {Chen }}$ on tensor products of symbols with non-integer partial sums of orders, i.e.:

$$
\phi_{r}^{\mathcal{R}}\left(\sigma_{1} \otimes \cdots \otimes \sigma_{k}\right)=f_{r}^{\text {Chen }} \sigma_{1} \otimes \cdots \otimes \sigma_{k}
$$

for any $\sigma_{i} \in \mathcal{S}^{\alpha_{i}, 0}, i=1, \ldots, k$ whenever the (left) partial sums of the orders $\alpha_{1}+$ $\alpha_{2}+\cdots+\alpha_{m}, m=1, \ldots, k$ are non-integer valued.

Proof: For convenience, we temporarily drop the subscript $r$. The Birkhoff decomposition [CK], M] for the minimal subtraction scheme reads:

$$
\Phi^{\mathcal{R}}=\left(\Phi_{-}^{\mathcal{R}}\right)^{\star-1} \star \Phi_{+}^{\mathcal{R}}
$$

where $\star$ is the convolution defined from the deconcatenation coproduct by $\phi \star \psi(\sigma)=$ $\phi(\sigma)+\psi(\sigma)+\sum_{(\sigma)} \phi\left(\sigma^{\prime}\right) \cdot \psi\left(\sigma^{\prime \prime}\right)$ with Sweedler's notations and where $\Phi_{+}^{\mathcal{R}}(\sigma)$ and $\Phi_{-}^{\mathcal{R}}(\sigma)$ are defined inductively on the degree of $\sigma \in \mathcal{T}\left(\mathcal{S}^{*, 0}\right)$. If $\pi$ denotes the projection onto the "pole part" $z^{-1} \mathbb{C}\left[z^{-1}\right]$ we have:

$$
\Phi_{-}^{\mathcal{R}}=-\pi\left[\Phi^{\mathcal{R}}+\sum_{(\sigma)} \Phi_{-}^{\mathcal{R}}\left(\sigma^{\prime}\right) \Phi^{\mathcal{R}}\left(\sigma^{\prime \prime}\right)\right], \quad \Phi_{+}^{\mathcal{R}}=(1-\pi)\left[\Phi^{\mathcal{R}}+\sum_{(\sigma)} \Phi_{-}^{\mathcal{R}}\left(\sigma^{\prime}\right) \Phi^{\mathcal{R}}\left(\sigma^{\prime \prime}\right)\right] .
$$

Since $\Phi_{+}^{\mathcal{R}}$ is also a character on $\left(\mathcal{T}\left(\mathcal{S}^{*, 0}\right), \amalg\right)$, it obeys the shuffle relation:

$$
\Phi_{+}^{\mathcal{R}}(\sigma \amalg \tau)(z)=\Phi_{+}^{\mathcal{R}}(\sigma)(z) \Phi_{+}^{\mathcal{R}}(\tau)(z)
$$

which holds as an equality of meromorphic functions holomorphic at $z=0$. Setting

$$
\phi^{\mathcal{R}}:=\Phi_{+}^{\mathcal{R}}(0)
$$


and applying the shuffle relation (32) at $z=0$ yields

$$
\phi^{\mathcal{R}}(\sigma \amalg \tau)=\phi^{\mathcal{R}}(\sigma) \phi^{\mathcal{R}}(\tau) .
$$

Now suppose that $\sigma=\sigma_{1} \otimes \cdots \otimes \sigma_{k}$ is such that none of the (left) partial sums of the orders $\alpha_{1}+\alpha_{2}+\cdots+\alpha_{m}, m=1, \ldots, k$ is an integer. Due to the particular form of the deconcatenation coproduct, the component $\sigma^{\prime}$ shares the same property (with $k$ replaced by the degree of $\sigma^{\prime}$ ). In other words, the linear span $\mathcal{K}$ of such $\sigma^{\prime}$ s is a right co-ideal of the coalgebra $\mathcal{T}\left(\mathcal{S}^{*, 0}\right)$. For any $\sigma \in \mathcal{K}$, the map $z \mapsto \Phi^{\mathcal{R}}(\sigma)(z)$ is holomorphic at 0 since poles only occur for values $z_{0}$ such that the partial orders lie in $\mathbb{Z}$. In particular $\pi\left(\Phi^{\mathcal{R}}(\sigma)\right)=0$, and it moreover shows for $k=1$ that:

$$
\Phi_{-}^{\mathcal{R}}(\sigma)=0 \text { and } \Phi_{+}^{\mathcal{R}}(\sigma)=\Phi^{\mathcal{R}}(\sigma) .
$$

On the grounds of the above recursive formulas for $\Phi_{-}^{\mathcal{R}}$ and $\Phi_{+}^{\mathcal{R}}$ we can then prove inductively on $k$ that (33) holds for any $k$, from which we infer that

$$
\phi^{\mathcal{R}}(\sigma)=\Phi^{\mathcal{R}}(\sigma)(0)=f^{\text {Chen }} \sigma_{1}(0) \otimes \cdots \otimes \sigma_{k}(0)=f^{\text {Chen }} \sigma_{1} \otimes \cdots \otimes \sigma_{k}
$$

since $\sigma_{i}(0)=\sigma_{i}$.

Motivated by this result we set the following definition.

Definition 6 Given a regularisation $\mathcal{R}: \tau \mapsto\{\tau(z)\}_{z \in W}$ on $\mathcal{S}^{*, 0}$, we define for any non-negative real number $r$ and any $\sigma_{1}, \ldots, \sigma_{k}$ the renormalised Chen integral of $\sigma_{1} \otimes \cdots \otimes \sigma_{k} b y$

$$
f_{r}^{\text {Chen, } \mathcal{R}} \sigma:=\phi_{r}^{\mathcal{R}}(\sigma)
$$

We illustrate this on an example which offers a continuous $n$-dimensional analog of regularised multiple zeta functions familiar to number theorists. For any $s \in \mathbb{R}$ we set for some non negative real number $v$

$$
\varphi_{s, v}(\xi)=\chi(\xi)(|\xi|+v)^{-s}
$$

where $\chi$ is some smooth cut-off function around 0 . For $\mu \in \mathbb{R}$ let $\gamma_{\mu}: \mathbb{C} \rightarrow \mathbb{C}$ be a holomorphic function such that $\gamma_{\mu}(z)=z+\mu z^{2}+o\left(z^{2}\right)$ for $z \rightarrow 0\left(\right.$ e.g. $\left.\gamma_{\mu}(z)=\frac{z}{1-\mu z}\right)$,

$$
\mathcal{R}_{\mu}: \sigma \mapsto(1-\chi) \sigma+\chi \sigma|\xi|^{-\gamma_{\mu}(z)}
$$

in which case

$$
\mathcal{R}_{\mu}\left(\varphi_{s}\right) \sim \varphi_{s+\gamma_{\mu}(z)}
$$

Applying the above construction with $r=0$ yields for non negative real numbers $v_{1}, \ldots, v_{k}$ renormalised quantities:

$$
\tilde{\zeta}_{n}^{\mu}\left(s_{1}, \ldots, s_{k} ; v_{1}, \ldots, v_{k}\right):=f^{\mathrm{Chen}, \mathcal{R}_{\mu}} \varphi_{s_{1}, v_{1}} \otimes \cdots \otimes \varphi_{s_{k}, v_{k}}
$$

When $v_{1}=\cdots=v_{k}=v$ we set

$$
\tilde{\zeta}_{n}^{\mu}\left(s_{1}, \ldots, s_{k} ; v\right)=\tilde{\zeta}_{n}^{\mu}\left(s_{1}, \ldots, s_{k} ; v_{1}, \ldots, v_{k}\right) .
$$


Corollary 1 For any $\mu \in \mathbb{R}, n \in \mathbb{N}$ and for any two words $u, v$ with letters in $\mathbb{C}$ we have:

$$
\tilde{\zeta}_{n}^{\mu}\left(u \amalg u^{\prime} ; v\right)=\tilde{\zeta}_{n}^{\mu}(u ; v) \cdot \tilde{\zeta}_{n}^{\mu}\left(u^{\prime} ; v\right) .
$$

Proof: This is a straightforward consequence of Theorem 2 applied to $\mathcal{R}_{\mu}$ and $\sigma_{i}=$ $\varphi_{s_{i}, v}$. 


\section{DISCRETE SUMS OF SYMBOLS}

We denote by $|$.$| the usual absolute value on \mathbb{R}$. The notation $\mathcal{P}^{\alpha, k}$ stands for positively supported symbols, i.e. symbols in $\mathcal{S}^{\alpha, k}(\mathbb{R})$ with support included in $] 0,+\infty[$. We keep mutatis mutandis the notations of subsection 1.1: in particular $\mathcal{P}^{*, 0}$ is a subalgebra of the filtered algebra $\mathcal{P}^{*, *}$.

We investigate in this second part a discrete counterpart of the results of part I. We will mostly stick to dimension $n=1$ : the reason for this lies in the crucial use of the Euler-MacLaurin formula. To the best of our knowledge a higher dimensional analogue, the Khovanskii-Pukhlikov formula (KSW2, GSW] exists only on polytopes, i.e. on balls with respect to norms which are not smooth outside the origin. The suitable framework for dealing with nested sums is then not clear. We return however to a higher dimensional situation at the end of the paper by considering radial functions with respect to the supremum norm $|\cdot|_{\infty}$ in $\mathbb{R}^{n}$, namely functions $\sigma=f \circ|\cdot|_{\infty}$ with $f \in \mathcal{P}^{*, *}$.

\subsection{Discrete sums versus integrals of symbols}

A Rota-Baxter operator on an algebra $A$ (defined, say over a field $k$ ) is a linear operator $P: A \rightarrow A$ such that the relation:

$$
P(f) P(g)=P(P(f) g+f P(g))-\theta P(f) P(g)
$$

holds for any $f, g \in A$. Here $\theta$ is a scalar in the field $k$ called the weight 6 . The operator $\tilde{P}: \mathcal{P}^{*, k} \rightarrow \mathcal{P}^{*, k+1}:$

$$
\tilde{P}(f)(\eta):=\int_{0}^{\eta} f(\xi) d \xi
$$

satisfies the weight zero Rota-Baxter relation. On the other hand the operator $P$ defined on sequences $f: \mathbb{N} \rightarrow \mathbb{C}$ by:

$$
P(f)(N)=\sum_{m=0}^{N} f(k)
$$

satisfies the Rota-Baxter relation of weight 1 , whereas the operator $Q=P$-Id satisfies the Rota-Baxter relation of weight -1 . We now want to compare the behaviour in the large of the continuous integrals $\tilde{P}(f)$ with that of discrete sums $P(f)$ when $f$ is a positively supported symbol (note that the sum actually begins with $m=1$ in this case). In order to compare $P(f)(N)$ and $\tilde{P}(f)(N)$ for large $N$ we make use of the Euler-MacLaurin formula ([Ha], [KSW1, see also [MP]). Formula (6) in KSW1] for $f \in \mathcal{P}^{*, *}$ yields:

$$
\begin{aligned}
P(f)(N)-\tilde{P}(f)(N) & =\frac{1}{2} f(N)+\sum_{k=2}^{2 K} \frac{B_{k}}{k !} f^{(k-1)}(N) \\
& +\frac{1}{(2 K+1) !} \int_{0}^{N} \overline{B_{2 K+1}}(x) f^{(2 K+1)}(x) d x .
\end{aligned}
$$

\footnotetext{
${ }^{6}$ Some authors use the opposite sign convention for the weight.
} 
with $\overline{B_{k}}(x)=B_{k}(x-[x])$. Here $B_{k}(x)=\sum_{i=0}^{k}\left(\begin{array}{c}k \\ i\end{array}\right) B_{k-i} x^{k}$ are the Bernoulli polynomials of degree $k$, the $B_{i}$ being the Bernoulli numbers, defined by the generating series:

$$
\frac{t}{e^{t}-1}=\sum_{i} \frac{B_{i}}{i !} t^{i}
$$

so that

$$
B_{0}=1, B_{1}=-\frac{1}{2}, B_{2}=\frac{1}{6}, B_{4}=-\frac{1}{30}, \ldots, \quad B_{2 j+1}=0 \text { for } j \geq 1 .
$$

Proposition 8 For any $f \in \mathcal{P}^{\alpha, k}$ and any $j \in \mathbb{N}$ chosen large enough the function $\bar{P}(f)$ defined by:

$$
\begin{aligned}
\bar{P}(f)(\eta) & :=\tilde{P}(f)(\eta)+\frac{1}{2} f(\eta)+\sum_{j=2}^{2 K} \frac{B_{j}}{j !} f^{(j-1)}(\eta) \\
& +\frac{1}{(2 K+1) !} \int_{0}^{\eta} \overline{B_{2 K+1}}(x) f^{(2 K+1)}(x) d x .
\end{aligned}
$$

lies in $\mathcal{P}^{\alpha+1, k+1}+\mathcal{P}^{0, k+1}$ and the difference $\bar{P}(f)-\tilde{P}(f)$ lies in $\mathcal{P}^{\alpha, k}$.

Remark 3 Note that when $\eta=N \in \mathbb{N}$ then $\bar{P}(f)(N)=P(f)(N)$. Hence $\bar{P}$ is an interpolation of $P$. Of course $\bar{Q}:=\bar{P}-\mathrm{Id}$ is an interpolation of $Q$ in the same sense.

Proof: The sum $\frac{1}{2} f(\eta)+\sum_{j=2}^{2 K} \frac{B_{j}}{j !} f^{(j-1)}(\eta)$ lies in $\mathcal{P}^{\alpha, k}$, whereas the integral $\tilde{P}(f)$ lies in $\mathcal{P}^{\alpha+1, k+1}+\mathcal{P}^{0, k+1}$. The result then follows from splitting the integral remainder term into $\int_{0}^{+\infty}(\ldots)-\int_{\eta}^{+\infty}(\ldots)$ : the first term in the sum is a constant for large enough $K$, and the second term is a symbol (with respect to the variable $\eta$ ) with arbitrarily small order $\alpha-(2 K+1)$ as $K$ grows, which lies in $\mathcal{P}^{\alpha, k}$.

We are now ready to introduce cut-off sums.

Corollary 2 For any $f \in \mathcal{P}^{*, *}$ and any positive integer $N$, the discrete $\operatorname{sum} \sum_{m=0}^{N} f(m)$ has an asymptotic expansion when $N \rightarrow \infty$ of the type (15) so that we can define the following cut-off sums as finite parts:

$$
\sum f:=\mathrm{fp}_{N \rightarrow \infty} \sum_{m=0}^{N} f(m)
$$

which coincides with $₹ f:=\mathrm{fp}_{N \rightarrow \infty} \sum_{m=0}^{N-1} f(m)$ whenever the order of $f$ does not belong to $\{0,1,2, \ldots\}$.

Proof: When $f \in \mathcal{P}^{\alpha, k}$ the maps $\eta \mapsto \bar{P}(f)(\eta)$ and $\eta \mapsto \bar{P}(f)(\eta)-f(\eta)$ lie in $\mathcal{P}^{*, k+1}$ and therefore have the expected asymptotic behaviour as $\eta \rightarrow \infty$. Setting $\eta=N$ and picking the constant term in the $N \rightarrow \infty$ expansion yields the cut-off sums $\sum f$ and $E f$. They a priori differ by $\operatorname{fp}_{N \rightarrow \infty} f(N)$ which vanishes whenever $f$ has order $\alpha \notin\{0,1,2, \ldots\}$. 
Proposition 9 Given a holomorphic regularisation $\mathcal{R}: h \mapsto h(z)$ on $\mathcal{P}^{*, k}$, for any $f \in \mathcal{P}^{*, k}$, the map

$$
z \mapsto f_{\mathbb{R}} f(z)-\sum f(z)
$$

is holomorphic for any $f \in \mathcal{P}^{*, k}$.

Consequently, the map $z \mapsto \sum f(z)$ is meromorphic with the same poles (of order $\leq k+1)$ as the map $z \mapsto f_{\mathbb{R}} f(z)$, which lie in the discrete set $\alpha^{-1}(\{-1,0,1,2, \ldots\})$ whenever $f(z)$ is a simple holomorphic family of order $\alpha(z)$. Moreover, $\sum f(z)$ and $\nexists f(z)$ coincide as meromorphic functions.

Proof: Let $f \mapsto f(z)$ be a holomorphic perturbation in $\mathcal{P}^{*, k}$. By Definition (38) in Proposition 8 , the difference $\operatorname{fp}_{N \rightarrow \infty} P(f(z))(N)-\mathrm{fp}_{N \rightarrow \infty} \tilde{P}(f(z))(N)$ is holomorphic. On the other hand, since the map $z \mapsto f f(z)=\operatorname{fp}_{N \rightarrow \infty} \tilde{P}(f(z))(N)$ is meromorphic with poles of order $\leq k+1$, the same property therefore holds for $z \mapsto$ $\mathrm{fp}_{N \rightarrow \infty} P(f(z))(N)=\sum f(z)$ and hence for $\sum f(z)$. When the regularisation is simple these poles lie in the discrete set $\alpha^{-1}(\{-1,0,1,2, \ldots\})$ where $\alpha(z)$ stands for the order of $f(z)$. Outside these poles, the sums $\sum f(z)$ and $₹ f(z)$ coincide so that they coincide as meromorphic functions.

On the grounds of this result we can set the following definition:

Definition 7 Given a holomorphic regularisation $\mathcal{R}: f \mapsto\{f(z)\}_{z \in W}$ on $\mathcal{P}^{*, k}$ we define for any $f \in \mathcal{P}^{*, k}$ the regularised sum

$$
\sum^{\mathcal{R}} f:=\operatorname{fp}_{z=0} \sum f(z)
$$

where by finite part we mean the constant term in the Laurent series expansion.

\subsection{The Riemann and Hurwitz zeta functions as regularised sums of a symbol}

Let $v$ be a non-negative real number. Riesz regularisation $\mathcal{R}: f(x) \mapsto f(x)|x|^{-z}$ applied to the symbol $f_{s ; v}: x \mapsto(x+v)^{-s}$ for any $\left.s \in\right] 0,+\infty$ [ gives rise to the Hurwitz $\zeta$-function 7 :

$$
\zeta(s ; v):=\sum^{\mathcal{R}} f_{s ; v}=\operatorname{fp}_{z=0} \sum_{n=1}^{\infty}(n+v)^{-s-z} .
$$

We recall the following elementary property of the Hurwitz zeta function on its domain of convergence:

$$
\zeta(s ; v+1)=\zeta(s ; v)-(v+1)^{-s} .
$$

In the following, we investigate its values together with the values of its derivatives

$$
\frac{d}{d v} \zeta(s ; v)=-s \zeta(s+1 ; v)
$$

at non-positive integer points.

\footnotetext{
${ }^{7}$ see Introduction for the notation convention
} 
Let us first recall the Euler-MacLaurin formula ([Ha], see also [C2, KSW1]). For any $f \in C^{\infty}\left(\mathbb{R}^{+}\right)$and for any integer $K \geq 0$,

$$
\begin{aligned}
\sum_{n=1}^{N} f(n) & =\int_{1}^{N} f(x) d x+\frac{f(1)+f(N)}{2}+\sum_{k=2}^{2 K} \frac{B_{k}}{k !}\left(f^{(k-1)}(N)-f^{(k-1)}(1)\right) \\
& +\frac{1}{(2 K+1) !} \int_{1}^{N} \overline{B_{2 K+1}}(x) f^{(2 K+1)}(x) d x
\end{aligned}
$$

with $\overline{B_{k}}(x)=B_{k}(x-[x])$ and where $B_{n}(x)=\sum_{k=0}^{n}\left(\begin{array}{l}n \\ k\end{array}\right) B_{n-k} x^{k}$ are the Bernoulli polynomials, the $B_{i}$ being the Bernoulli numbers. For any $a \in \mathbb{R}$ and $m \in \mathbb{N}-\{0\}$ we use the notation:

$$
[a]_{m}:=a(a-1) \cdots(a-m+1)
$$

which should not lead to any confusion with the integer part $[a]$ of $a$. The following proposition yields back the known Hurwitz zeta values at non positive integers (see e.g. [C2]) as well as their derivatives w.r. to the parameter.

Proposition 10 Let $z \mapsto \gamma(z)$ be a holomorphic function with $\gamma^{\prime}(0)=1$. Let $\lambda \in$ $\mathbb{C}-\{0\}$. The map $z \mapsto \sum_{n=1}^{\infty}(n+v)^{a-\lambda \gamma(z)}$ is holomorphic at zero for any $a \neq-1$. For any $a \in \mathbb{N}$ and any non negative real $v$ the Hurwitz zeta value $\zeta(-a ; v)=-\frac{B_{a+1}(1+v)}{a+1}$ can be obtained as:

$$
\zeta(-a ; v)=\operatorname{fp}_{z=0} \sum_{n=1}^{\infty}(n+v)^{a-\lambda \gamma(z)}=\lim _{z \rightarrow 0} \sum_{n=1}^{\infty}(n+v)^{a-\lambda \gamma(z)} .
$$

In particular it is a rational number when $v$ is rational. When $a=-1$ the residue at 0 which reads $\frac{1}{\lambda}$, is independent of $v$. Similar formulae hold for the derivatives at non positive integers:

$$
\begin{aligned}
\frac{d}{d v} \zeta(-a ; v) & =a \zeta(-a ; v) \quad \text { if } \quad a \neq 0 \\
& =-1 \quad \text { if } \quad a=0 .
\end{aligned}
$$

Proof: Applied to $f(x)=(x+v)^{a}$ with $a \in \mathbb{C}$, the Euler-MacLaurin formula gives:

$$
\begin{gathered}
\sum_{0<n \leq N}(n+v)^{a}=\frac{(N+v)^{a}+(1+v)^{a}}{2}+\int_{1}^{N}(x+v)^{a} d x \\
+\sum_{k=2}^{2 K} B_{k} \frac{[a]_{k-1}}{k !}\left((N+v)^{a-k+1}-(1+v)^{a-k+1}\right)+\frac{[a]_{2 K+1}}{(2 K+1) !} \int_{1}^{N} \overline{B_{2 K+1}}(x)(x+v)^{a-2 K-1} d x \\
=\left(1-\delta_{a+1}\right) \frac{(N+v)^{a+1}}{a+1}-\left(1-\delta_{a+1}\right) \frac{(1+v)^{a+1}}{a+1}+\frac{(N+v)^{a}+(1+v)^{a}}{2}+\delta_{a+1}(\log (N+v)-\log (1+v)) \\
+\sum_{k=2}^{2 K} B_{k} \frac{[a]_{k-1}}{k !}\left((N+v)^{a-k+1}-(1+v)^{a-k+1}\right)+\frac{[a]_{2 K+1}}{(2 K+1) !} \int_{1}^{N} \overline{B_{2 K+1}}(x)(x+v)^{a-2 K-1} d x . \quad(42)
\end{gathered}
$$

Let us set

$$
R_{K}(a):=\frac{[a]_{2 K+1}}{(2 K+1) !} \int_{1}^{N} \overline{B_{2 K+1}}(x)(x+v)^{a-2 K-1} d x ; \quad S_{K}(a):=\sum_{k=2}^{2 K} B_{k} \frac{[a]_{k-1}}{k !}(1+v)^{a-k+1} .
$$


Replacing $a$ in (42) by $a-\lambda \gamma(z)$ and taking finite parts as $N \rightarrow \infty$ we have:

$\sum_{1}^{\infty}(n+v)^{a-\lambda \gamma(z)}=-\frac{(1+v)^{a-\lambda \gamma(z)+1}}{a-\lambda \gamma(z)+1}+\frac{(1+v)^{a-\lambda \gamma(z)}}{2}-S_{K}(a-\lambda \gamma(z))+R_{K}(a-\lambda \gamma(z))$.

Hence $\operatorname{Res}_{z=0} \sum_{1}^{\infty}(n+v)^{a-\lambda \gamma(z)}=\delta_{a+1} \frac{1}{\lambda}$. Taking the finite part at 0 then yields:

$\mathrm{fp}_{z=0} \sum_{1}^{\infty}(n+v)^{a-\lambda \gamma(z)}=-\left(1-\delta_{a+1}\right) \frac{(1+v)^{a+1}}{a+1}-\delta_{a+1} \log (1+v)+\frac{(1+v)^{a}}{2}-S_{K}(a)+R_{K}(a)$,

which for a non negative integer $a$ gives:

$$
\lim _{z \rightarrow 0} \sum_{0}^{\infty}(n+v)^{a-\lambda \gamma(z)}=-\sum_{k=0}^{a+1}\left(\begin{array}{c}
a+1 \\
k
\end{array}\right) B_{k}(1+v)^{a+1}=-\frac{B_{a+1}(1+v)}{a+1} .
$$

We now turn to derivatives. Since $\frac{d}{d v} \zeta(s ; v)=-s \zeta(s+1 ; v)$, at a non negative integer $a$ we have:

$$
\begin{aligned}
\frac{d}{d v} \zeta(-a ; v) & =\operatorname{fp}_{z=0}\left((a-\lambda \gamma(z)) \sum_{1}^{\infty}(n+v)^{a-\lambda \gamma(z)}\right. \\
& =a \operatorname{fp}_{z=0} \sum_{1}^{\infty}(n+v)^{a-\lambda \gamma(z)} \quad \text { if } \quad a \neq 0 \\
& =-\lambda \operatorname{Res}_{z=0} \sum_{1}^{\infty}(n+v)^{a-\lambda \gamma(z)} \text { if } a=0
\end{aligned}
$$

so that

$$
\begin{aligned}
\frac{d}{d v} \zeta(-a ; v) & =a \zeta(-a ; v) \quad \text { if } \quad a \neq 0 \\
& =-\lambda \frac{1}{\lambda}=-1 \quad \text { if } \quad a=0
\end{aligned}
$$




\section{NESTED SUMS OF SYMBOLS}

\subsection{The Hoffman isomorphism and stuffle relations for discrete nested sums}

We recall here the explicit construction by M. Hoffman ([H2]) of an isomorphism between the stuffle Hopf algebra and the shuffle Hopf algebra.

Definition 8 Let $k, l, r \in \mathbb{N}$ with $k+l-r>0$. A $(k, l)$-quasi-shuffle of type $r$ is a surjective map $\pi$ from $\{1, \ldots, k+l\}$ onto $\{1, \ldots, k+l-r\}$ such that $\pi(1)<\cdots<\pi(k)$ and $\pi(k+1)<\cdots<\pi(k+l)$. We shall denote by $\operatorname{mix} \operatorname{sh}(k, l ; r)$ the set of $(k, l)-$ quasi-shuffles of type $r$. The elements of mix $\operatorname{sh}(k, l ; 0)$ are the ordinary $(k, l)$-shuffles. Quasi-shuffles are also called mixable shuffles or stuffles. We denote by $\operatorname{mix} \operatorname{sh}(k, l)$ the set of $(k, l)$-quasi-shuffles (of any type).

Let $(\mathcal{A}, \bullet)$ be a commutative (not necessarily unital) algebra. Let $\Delta$ be the deconcatenation coproduct on $\mathcal{T}(\mathcal{A})=\bigoplus_{k \geq 0} \mathcal{A}^{\otimes k}$, let $\amalg$ be the shuffle product, and let $\star_{\bullet}$ the product on $\mathcal{T}(\mathcal{A})$ defined by:

$$
\left(v_{1} \otimes \cdots \otimes v_{k}\right) \star_{\bullet}\left(v_{k+1} \otimes \cdots \otimes v_{k+l}\right)=\sum_{\pi \in \operatorname{mix} \operatorname{sh}(k, l)} w_{1}^{\pi} \otimes \cdots \otimes w_{k+l-r}^{\pi},
$$

with :

$$
w_{j}^{\pi}=\prod_{i \in\{1, \ldots, k+l\}, \pi(i)=j} v_{i} .
$$

(the product above is the product $\bullet$ of $\mathcal{A}$, and contains only one or two terms).

Theorem 3 (M. Hoffman, [H2] theorems 3.1 and 3.3)

- $\left(\mathcal{T}(\mathcal{A}), \star_{\bullet}, \Delta\right)$ is a commutative connected filtered Hopf algebra.

- There is an isomorphism of Hopf algebras :

$$
\exp :(T(\mathcal{A}), \amalg, \Delta) \stackrel{\sim}{\longrightarrow}\left(T(\mathcal{A}), \star_{\bullet}, \Delta\right) .
$$

M. Hoffman in $\mathrm{H} 2$ gives a detailed proof in a slightly more restricted context, which can be easily adapted in full generality (see also EG]). Hoffman's isomorphism is built explicitly as follows: let $\mathcal{P}(n)$ be the set of compositions of the integer $n$, i.e. the set of sequences $I=\left(i_{1}, \ldots, i_{k}\right)$ of positive integers such that $i_{1}+\cdots+i_{k}=n$. For any $u=v_{1} \otimes \cdots \otimes v_{n} \in T(\mathcal{A})$ and any composition $I=\left(i_{1}, \ldots, i_{k}\right)$ of $n$ we set:

$$
I[u]:=\left(v_{1} \bullet \cdots \bullet v_{i_{1}}\right) \otimes\left(v_{i_{1}+1} \bullet \cdots \bullet v_{i_{1}+i_{2}}\right) \otimes \cdots \otimes\left(v_{i_{1}+\cdots+i_{k-1}+1} \bullet \cdots \bullet v_{n}\right) .
$$

We then further define:

$$
\exp u=\sum_{I=\left(i_{1}, \ldots, i_{k}\right) \in \mathcal{P}(n)} \frac{1}{i_{1} ! \cdots i_{k} !} I[u]
$$

Moreover ( $\underline{\mathrm{H} 2}$, lemma 2.4), the inverse log of exp is given by :

$$
\log u=\sum_{I=\left(i_{1}, \ldots, i_{k}\right) \in \mathcal{P}(n)} \frac{(-1)^{n-k}}{i_{1} \cdots i_{k}} I[u] .
$$


For example for $v_{1}, v_{2}, v_{3} \in \mathcal{A}$ we have :

$$
\begin{array}{rll}
\exp v_{1}=v_{1} & , \quad \log v_{1}=v_{1}, \\
\exp \left(v_{1} \otimes v_{2}\right)=v_{1} \otimes v_{2}+\frac{1}{2} v_{1} \bullet v_{2} & , \quad \log \left(v_{1} \otimes v_{2}\right)=v_{1} \otimes v_{2}-\frac{1}{2} v_{1} \bullet v_{2}, \\
\exp \left(v_{1} \otimes v_{2} \otimes v_{3}\right)=v_{1} \otimes v_{2} \otimes v_{3} & +\frac{1}{2}\left(v_{1} \bullet v_{2} \otimes v_{3}+v_{1} \otimes v_{2} \bullet v_{3}\right)+\frac{1}{6} v_{1} \bullet v_{2} \bullet v_{3}, \\
\log \left(v_{1} \otimes v_{2} \otimes v_{3}\right)=v_{1} \otimes v_{2} \otimes v_{3} & -\frac{1}{2}\left(v_{1} \bullet v_{2} \otimes v_{3}+v_{1} \otimes v_{2} \bullet v_{3}\right)+\frac{1}{3} v_{1} \bullet v_{2} \bullet v_{3} .
\end{array}
$$

Let $V$ be the space of sequences $f: \mathbb{N} \rightarrow \mathbb{C}$ such that $f(0)=0$. It is a commutative algebra for the ordinary product.

Theorem 4 1. Consider the commutative algebra $(V, \bullet)$ where $\bullet$ is the ordinary product. For any $N \in \mathbb{N}$, the truncated discrete nested sums on $\mathcal{T}(V)$ defined by:

$$
\sum_{<}^{N, \text { Chen }} f_{1} \otimes \cdots \otimes f_{k}:=\sum_{0<n_{k}<\cdots<n_{1}<N} f_{1}\left(n_{1}\right) \cdots f_{k}\left(n_{k}\right)
$$

for any $f=f_{1} \otimes \cdots \otimes f_{k} \in V^{\otimes k}$ fulfill the following relations:

$$
\sum_{<}^{N, \text { Chen }} f \star_{\bullet} g=\left(\sum_{<}^{N, \text { Chen }} f\right)\left(\sum_{<}^{N, \text { Chen }} g\right) .
$$

2. Whenever the nested sums converge as $N \rightarrow \infty$, we have in the limit:

$$
\sum_{<}^{\text {Chen }} f \star_{\bullet} g=\left(\sum_{<}^{\text {Chen }} f\right)\left(\sum_{<}^{\text {Chen }} g\right) .
$$

3. The same statements hold with the weak inequality version:

$$
\sum_{\leq}^{N, \text { Chen }} f_{1} \otimes \cdots \otimes f_{k}:=\sum_{0<n_{k} \leq \cdots \leq n_{1} \leq N} f_{1}\left(n_{1}\right) \cdots f_{k}\left(n_{k}\right)
$$

provided $\bullet$ is now the opposite of the ordinary product, i.e. $v_{1} \bullet v_{2}=-v_{1} v_{2}$.

Proof: It is enough to prove the theorem for $f=f_{1} \otimes \cdots \otimes f_{k} \in V^{\otimes k}$ and $g=f_{k+1} \otimes \cdots \otimes f_{k+l} \in V^{\otimes l}$. Let us prove the theorem for the weak inequality case. The domain:

$$
P_{k, l}:=\left\{n_{1}>\cdots>n_{k}>0\right\} \times\left\{n_{k+1}>\cdots>n_{k+l}>0\right\} \subset(\mathbb{N}-\{0\})^{k+l}
$$

is partitioned into:

$$
P_{k, l}=\coprod_{\pi \in \operatorname{mix} \operatorname{sh}(k, l)} P_{\pi}
$$

where the domain $P_{\pi}$ is defined by:

$$
P_{\pi}=\left\{\left(n_{1}, \ldots, n_{k+l}\right) / n_{\pi_{m}}>n_{\pi_{p}} \text { if } m>p \text { and } \pi_{m} \neq \pi_{p} \text {, and } n_{m}=n_{p} \text { if } \pi_{m}=\pi_{p}\right\} \text {. }
$$

As we must replace strict inequalities by large ones, let us consider the "closures"

$$
\overline{P_{\pi}}:=\left\{\left(n_{1}, \ldots, n_{k+l}\right) / n_{\pi_{m}} \geq n_{\pi_{p}} \text { if } m \geq p \text { and } n_{m}=n_{p} \text { if } \pi_{m}=\pi_{p}\right\} .
$$


which then overlap. By the inclusion-exclusion principle we have:

$$
\overline{P_{k, l}}=\coprod_{0 \leq r \leq \min (k, l)}(-1)^{r} \coprod_{\pi \in \operatorname{mix} \operatorname{sh}(k, l ; r)} \overline{P_{\pi}},
$$

where we have set:

$$
\overline{P_{k, l}}:=\left\{n_{1} \geq \cdots \geq n_{k}>0\right\} \times\left\{n_{k+1} \geq \cdots \geq n_{k+l}>0\right\} \subset(\mathbb{N}-\{0\})^{k+l}
$$

Each term in equation (46) must be added if $r$ is even, and removed if $r$ is odd. Considering the summation of $f_{1} \otimes \cdots \otimes f_{k+l}$ over each $\overline{P_{\pi}}$, this decomposition immediately yields the equality:

$$
\begin{aligned}
& \left(\sum_{0 \leq n_{k} \leq \cdots \leq n_{1} \leq N} f_{1}\left(n_{1}\right) \cdots f_{i}\left(n_{k}\right)\right)\left(\sum_{0 \leq n_{k+l} \leq \cdots \leq n_{k+1} \leq N} f_{k+1}\left(n_{k+1}\right) \cdots f_{k+l}\left(n_{k+l}\right)\right) \\
= & \sum_{\leq}^{N, \text { Chen }} \sum_{\pi \in \operatorname{mix} \operatorname{sh}(k, l)} f^{\pi},
\end{aligned}
$$

where $f^{\pi}=f_{1}^{\pi} \otimes \cdots \otimes f_{k+l-r}^{\pi}$ is the tensor product defined by:

$$
f_{j}^{\pi}=\underset{i \in\{1, \ldots, k+l\}, \pi(i)=j}{\bullet} f_{i} .
$$

The stuffle relations (44) are then a re-writing of equality (47) using the commutative algebra $(V, \bullet)$. Taking the limit as $N \rightarrow \infty$ provides the second statement of the theorem. The proof is similar for the strict inequality case, using the domains $P_{\pi}$ rather than the "closures" $\overline{P_{\pi}}$. As there are no overlaps the signs disappear in the formula (46).

\subsection{Cut-off nested sums of symbols and multiple (Hurwitz) zeta functions}

In this section we iterate the interpolated summation operators $\bar{P}$ and $\bar{Q}$ defined on $\mathcal{P}^{*, *}$ by (38) in a way to be made precise. As a consequence of Proposition 8 we derive the following result.

Proposition 11 Given $\sigma_{i} \in \mathcal{P}^{\alpha_{i}, 0}, i=1, \ldots, k$, setting $\sigma:=\sigma_{1} \otimes \cdots \otimes \sigma_{k}$, the function $\widetilde{\sigma}$ defined by:

$$
\widetilde{\sigma}:=\sigma_{1} \bar{P}\left(\cdots \sigma_{k-2} \bar{P}\left(\sigma_{k-1} \bar{P}\left(\sigma_{k}\right)\right) \cdots\right)
$$

lies in $\mathcal{P}^{*, k-1}$ as a linear combination of (positively supported) symbols in $\mathcal{P}^{\alpha_{1}+\cdots+\alpha_{m}+m-1, m-1}$, $m \in\{1, \ldots, k\}$. It has real order $\omega_{1}$ as defined in Lemma 圆. The same holds for the function $(\widetilde{\sigma})^{\prime}$ defined the same way with $\bar{Q}=\bar{P}-\operatorname{Id}$ instead of $\bar{P}$.

Proof: Let us first observe that by Proposition 8 given two symbols $\tau_{1} \in \mathcal{P}^{\beta_{1}, j_{1}}$ and $\tau_{2} \in \mathcal{P}^{\beta_{2}, j_{2}}$, the expression $\tau_{1} \bar{P}\left(\tau_{2}\right)$ lies in $\mathcal{P}^{*, j_{1}+j_{2}+1}$ as a linear combination of symbols in $\mathcal{P}^{\beta_{1}, j_{1}}$ and $\mathcal{P}^{\beta_{1}+\beta_{2}+1, j_{1}+j_{2}+1}$. In particular, setting $\beta_{i}=\alpha_{i}$ and $j_{1}=j_{2}=0$ shows the proposition holds when $k=2$.

The statement for $k>2$ then follows from an induction procedure on $k$. Assuming 
that $\tau_{2}:=\sigma_{2} \bar{P}\left(\cdots \sigma_{k-2} \bar{P}\left(\sigma_{k-1} \bar{P}\left(\sigma_{k}\right)\right) \ldots\right)$ lies in $\mathcal{P}^{*, k-2}$ as a linear combination of logpolyhomogeneous symbols $\tau_{2, m}$ in $\mathcal{P}^{\alpha_{2}+\cdots+\alpha_{m}+m-2, m-2}, m$ varying in $\{2, \ldots, k\}$, we infer from our preliminary observation applied to $\tau_{1}=\sigma_{1}$ and each $\tau_{2, m}$ that $\sigma_{1} \bar{P}\left(\tau_{2}\right)$ lies in $\mathcal{P}^{*, k-1}$ as a linear combination of log-polyhomogeneous symbols $\sigma_{1} \bar{P}\left(\tau_{2, m}\right)$ in $\mathcal{P}^{\alpha_{1}+\alpha_{2}+\cdots+\alpha_{m}+m-1, m-1}, m \in\{1, \ldots, k\}$.

We are now ready to define discrete nested sums of symbols. Combining Propositions 11 and 8 shows that the cut-off sums of the symbols $\widetilde{\sigma}$ and $(\widetilde{\sigma})^{\prime}$ are well defined so that we can set the following definitions.

Definition 9 For $\sigma_{1}, \ldots, \sigma_{k} \in \mathcal{P}^{*, 0}$ and $\sigma:=\sigma_{1} \otimes \cdots \otimes \sigma_{k}$ we call

$$
\sum_{\leq}^{\text {Chen }} \sigma:=\sum_{n \in \mathbb{N}} \widetilde{\sigma}(n)=\sum_{0<n_{k} \leq \cdots \leq n_{1}} \sigma_{1}\left(n_{1}\right) \cdots \sigma_{k}\left(n_{k}\right),
$$

the cut-off nested sum of $\sigma=\sigma_{1} \otimes \cdots \otimes \sigma_{k}$. The strict inequality version is defined by:

$$
\sum_{<}^{\text {Chen }} \sigma:=\sum_{n \in \mathbb{N}}(\widetilde{\sigma})^{\prime}(n)=\sum_{0<n_{k}<\cdots<n_{1}} \sigma_{1}\left(n_{1}\right) \cdots \sigma_{k}\left(n_{k}\right) .
$$

Remark 4 This definition of cut-off nested sums (in its weak inequality version) matches with the one given in [MP] paragraph 6.3. The restriction to positively supported symbols allows us to drop the absolute values here.

Corollary 3 The discrete nested sums above converge whenever $\operatorname{Re}\left(\alpha_{1}+\cdots+\alpha_{m}\right)<$ $-m$ for any $m \in\{1, \ldots, k\}$ in which case they are ordinary discrete nested sums:

$$
\begin{aligned}
\sum_{\leq}^{\text {Chen }} \sigma & =\sum_{0<n_{k} \leq \cdots \leq n_{1}} \sigma_{1}\left(n_{1}\right) \otimes \cdots \otimes \sigma_{k}\left(n_{k}\right) \\
\text { and } \quad \sum_{<}^{\text {Chen }} \sigma & =\sum_{0<n_{k}<\cdots<n_{1}} \sigma_{1}\left(n_{1}\right) \otimes \cdots \otimes \sigma_{k}\left(n_{k}\right)
\end{aligned}
$$

Proof: By Proposition 8 , the orders of $\widetilde{\sigma}=\sigma_{1} \bar{P}\left(\cdots \sigma_{k-2} \bar{P}\left(\sigma_{k-1} \bar{P}\left(\sigma_{k}\right)\right) \ldots\right)$ and $\widetilde{\sigma}^{\prime}=$ $\sigma_{1} \bar{Q}\left(\cdots \sigma_{k-2} \bar{Q}\left(\sigma_{k-1} \bar{Q}\left(\sigma_{k}\right)\right) \ldots\right)$ coincide with that of $\sigma^{\text {Chen }}=\sigma_{1} \tilde{P}\left(\cdots \sigma_{k-2} \tilde{P}\left(\sigma_{k-1} \tilde{P}\left(\sigma_{k}\right)\right) \ldots\right)$. Since the latter is smaller than -1 under the assumptions of the corollary, the convergence follows from the definition of the nested sums.

In the latter case, we drop the bar across the summation sign and write $\sum_{\leq}^{\text {Chen }} \sigma$ and $\sum_{<}^{\text {Chen }} \sigma$. Let us now apply the above results to $\sigma_{i}(\xi):=\sigma_{s_{i}, v_{i}}(\xi):=\left(\xi+v_{i}\right)^{-s_{i}} \chi(x)$, where $s_{1}, \ldots, s_{k}$ are complex numbers and $v_{1}, \ldots, v_{k}$ non negative real numbers. Here $\chi$ is a cut-off function which vanishes on $]-\infty, \varepsilon[$ with $\varepsilon>0$ and such that $\chi(\xi)=1$ for $|\xi| \geq 1$. This will give back the multiple zeta functions familiar to number theorists as well as multiple Hurwitz zeta functions which provide a multiple analog of ordinary Hurwitz zeta functions (see e.g. C2 8 .

\footnotetext{
${ }^{8}$ The case $k=1$ gives back the Hurwitz zeta function $\zeta(s, v+1)$. We choose these conventions in order to deal with multiple zeta and multiple Hurwitz zeta functions simultaneously.
} 
Theorem 5 If $\operatorname{Re}\left(s_{1}+\cdots+s_{m}\right)>m$ for any $m \in\{1, \ldots, k\}$ the discrete nested sums:

$\zeta\left(s_{1}, \ldots, s_{k} ; v_{1}, \ldots, v_{k}\right):=\sum_{<}^{\text {Chen }} \sigma_{s_{1}, v_{1}} \otimes \cdots \otimes \sigma_{s_{k}, v_{k}}=\sum_{1 \leq n_{k}<n_{k-1}<\cdots<n_{1}}\left(n_{k}+v_{k}\right)^{-s_{k}} \cdots\left(n_{1}+v_{1}\right)^{-s_{1}}$

for non negative $v_{1}, \ldots, v_{k}$ converge and extend meromorphically to all $s_{i} \in \mathbb{C}$ by the cut-off nested sum:

$$
\zeta\left(s_{1}, \ldots, s_{k} ; v_{1}, \ldots, v_{k}\right):=\sum_{<}^{\text {Chen }} \sigma_{s_{1}, v_{1}} \otimes \cdots \otimes \sigma_{s_{k}, v_{k}} .
$$

Setting $v_{1}=\cdots=v_{k}=0$ gives similar statements for multiple zeta functions

$$
\zeta\left(s_{1}, \ldots, s_{k}\right):=\sum_{<}^{\text {Chen }} \sigma_{s_{1}} \otimes \cdots \otimes \sigma_{s_{k}} .
$$

A similar statement holds for the weak inequality version

$\bar{\zeta}\left(s_{1}, \ldots, s_{k} ; v_{1}, \ldots, v_{k}\right):=\sum_{\leq}^{\text {Chen }} \sigma_{s_{1}, v_{1}} \otimes \cdots \otimes \sigma_{s_{k}, v_{k}}=\sum_{1 \leq n_{k} \leq n_{k-1} \leq \cdots \leq n_{1}}\left(n_{k}+v_{k}\right)^{-s_{k}} \cdots\left(n_{1}+v_{1}\right)^{-s_{1}}$.

Proof: The existence of the extension follows immediately from applying the results of Corollary 3 to $\sigma_{i}=\sigma_{s_{i}, v_{i}}$ of order $-s_{i}$. We postpone the proof of meromorphicity to the next section (Corollary 4 ).

We have the following relations between both versions (see $[\underline{\mathrm{H}}$ ):

$$
\begin{aligned}
\bar{\zeta}\left(a_{1}, \ldots, a_{k} ; v_{1}, \ldots, v_{k}\right) & =\sum_{I=\left(i_{1}, \ldots, i_{r}\right) \in \mathcal{P}(k)} \zeta\left(b_{1}^{I}, \ldots b_{r}^{I} ; v_{1}, \ldots, v_{r}\right), \\
\zeta\left(a_{1}, \ldots, a_{k} ; v_{1}, \ldots, v_{k}\right) & =\sum_{I=\left(i_{1}, \ldots, i_{r}\right) \in \mathcal{P}(k)}(-1)^{k-r} \bar{\zeta}\left(b_{1}^{I}, \ldots b_{r}^{I} ; v_{1}, \ldots, v_{r}\right)
\end{aligned}
$$

with $b_{s}^{I}:=a_{i_{1}+\cdots+i_{s-1}+1}+\cdots+a_{i_{1}+\cdots+i_{s}}$. For any multi-index $\alpha=\left(\alpha_{1}, \ldots, \alpha_{k}\right)$ we have the following identity between meromorphic functions of $\left(s_{1}, \ldots, s_{k}\right)$ :

$\partial_{v_{1}}^{\alpha_{1}} \ldots \partial_{v_{k}}^{\alpha_{k}} \zeta\left(s_{1}, \ldots, s_{k} ; v_{1}, \ldots, v_{k}\right)=(-1)^{|\alpha|} s_{1}^{\alpha_{1}} \ldots s_{k}^{\alpha_{k}} \zeta\left(s_{1}+\alpha_{1}, \ldots, s_{k}+\alpha_{k} ; v_{1}, \ldots, v_{k}\right)$.

Higher-dimensional analogues of multiple zeta functions are discussed in Section 5 Let us also mention the following vanishing result:

Lemma 3 For any non-negative integers $a_{1}, \ldots, a_{k}$ and any rational numbers $v_{1}, \ldots v_{k}$, the expression

$$
\sum_{0<n_{k}<\cdots<n_{1} \leq N}\left(n_{1}+v_{1}\right)^{a_{1}} \cdots\left(n_{k}+v_{k}\right)^{a_{k}}
$$

is a polynomial expression in $N$ with rational coefficients. The following corresponding cut-off sum vanishes:

$$
\mathrm{fp}_{N \rightarrow \infty} \sum_{0<n_{k}<\cdots<n_{1} \leq N}\left(n_{1}+v_{1}\right)^{a_{1}} \cdots\left(n_{k}+v_{k}\right)^{a_{k}}=0 .
$$


Proof: First observe that once we know that the expression:

$$
f(N):=\sum_{0<n_{k}<\cdots<n_{1} \leq N}\left(n_{1}+v_{1}\right)^{a_{1}} \cdots\left(n_{k}+v_{k}\right)^{a_{k}}
$$

is a polynomial expression in $N$, then its finite part as $N \rightarrow+\infty$ corresponds to its value at $N=0$. Next, for any rational $v$ the polynomials $D_{m}^{v}: N \mapsto(N+v)^{m}-$ $(N+v-1)^{m}, m \geq 1$ form a basis of the vector space $\mathbb{Q}[N]$. This means that for any polynomial $P \in \mathbb{Q}[N]$ there is a polynomial $Q \in \mathbb{Q}[N]$ (defined up to an additive constant) such that $P(N)=Q(N+v)-Q(N+v-1)$. We obviously get for $N \geq 1$ :

$$
\sum_{n=1}^{N} P(n)=Q(N+v)-Q(v)
$$

hence a polynomial expression in $N$ with rational coefficients, which vanishes at $N=0$. The lemma follows then by induction on the depth $k$, by writing:

$$
f(N)=\sum_{n_{1}=1}^{N}\left(n_{1}+v\right)^{a_{1}}\left(\sum_{0<n_{k}<\cdots<n_{2}<n_{1}}\left(n_{2}+v\right)^{a_{2}} \cdots\left(n_{k}+v\right)^{a_{k}}\right),
$$

since the expression between parentheses is in $\mathbb{Q}\left[n_{1}\right]$ by the induction assumption.

\subsection{Renormalised nested sums of symbols}

In this section we mimic the construction of renormalised nested integrals of symbols carried out previously under the requirement that they obeyed shuffle relations. Via the Euler-MacLaurin formula, which provides a bridge to nested sums, we can similarly build meromorphic families of nested sums of symbols on $\mathbb{R}$.

Theorem 6 Let $W \subset \mathbb{C}$ be an open neighbourhood of 0 . Let $\sigma_{1}, \ldots, \sigma_{l} \in \mathcal{P}^{*, 0}$, and simple holomorphic perturbations $\mathcal{R}_{1}\left(\sigma_{1}\right)(z), \ldots, \mathcal{R}_{l}\left(\sigma_{l}\right)(z) \in \mathcal{P}^{*, 0}$ with holomorphic orders $\alpha_{1}(z), \ldots, \alpha_{l}(z), z \in W$, such that $\operatorname{Re}\left(\alpha_{1}^{\prime}(z)+\cdots+\alpha_{m}^{\prime}(z)\right)<0$ for any $m \in\{1, \ldots, m\}$ and for any $z \in W$. Let $\sigma:=\sigma_{1} \otimes \cdots \otimes \sigma_{l}$ and $\sigma(z):=\sigma_{1}(z) \otimes \cdots \otimes \sigma_{l}(z)$ where we have set $\sigma_{i}(z):=\mathcal{R}_{i}\left(\sigma_{i}\right)(z)$. Then

1. The maps

$$
\left(z_{1}, \ldots, z_{k}\right) \mapsto \sum_{<}^{\text {Chen }} \sigma_{1}\left(z_{1}\right) \otimes \cdots \otimes \sigma_{l}\left(z_{k}\right)
$$

are meromorphic with poles on a countable number of hypersurfaces

$$
\sum_{i=1}^{j} \alpha_{i}\left(z_{i}\right) \in-j+\mathbb{N}
$$

of multiplicity $j$ varying in $\{1, \ldots, k\}$.

2. the maps $z \mapsto \sum_{\leq}^{\text {Chen }} \sigma(z)$ and $z \mapsto \sum_{<}^{\text {Chen }} \sigma(z)$ are meromorphic on $W$ with poles of order $\leq k$. 
3. If $\operatorname{Re}\left(\alpha_{1}+\cdots+\alpha_{j}\right)<-j$ for any $j \in\{1, \ldots, k\}$, the nested sums converge and boil down to ordinary nested sums (independently of the perturbation):

$$
\begin{aligned}
\sum_{<}^{\text {Chen, } \mathcal{R}} \sigma & =\lim _{z \rightarrow 0} \sum_{<}^{\text {Chen }} \sigma(z)=\sum_{<}^{\text {Chen }} \sigma, \\
\sum_{\leq}^{\text {Chen, } \mathcal{R}} \sigma & =\lim _{z \rightarrow 0} \sum_{\leq}^{\text {Chen }} \sigma(z)=\sum_{\leq}^{\text {Chen }} \sigma .
\end{aligned}
$$

These convergent nested sums moreover obey stuffle relations:

$$
\begin{aligned}
& \sum_{<}^{\text {Chen }} \sigma \star_{\bullet} \tau=\sum_{<}^{\text {Chen }} \sigma \cdot \sum_{<}^{\text {Chen }} \tau, \\
& \sum_{\leq}^{\text {Chen }} \sigma \star_{\bullet} \tau=\sum_{\leq}^{\text {Chen }} \sigma \cdot \sum_{\leq}^{\text {Chen }} \tau,
\end{aligned}
$$

where - stands for the ordinary product in the first case, and stands for the opposite of the ordinary product in the second case.

Proof: We give the proof in the weak inequality case since the strict inequality case is similar.

1. With the notations of Proposition 11 and setting $\sigma\left(\underline{z}_{k}\right):=\sigma_{1}\left(z_{1}\right) \otimes \cdots \otimes \sigma_{k}\left(z_{k}\right)$ we have

$$
\sum_{\leq}^{\text {Chen }} \sigma\left(\underline{z}_{k}\right)=\sum \widetilde{\sigma\left(\underline{z}_{k}\right)}=\operatorname{fp}_{N \rightarrow \infty} \bar{P}\left(\widetilde{\sigma\left(\underline{z}_{k}\right)}\right)(N) .
$$

By Proposition 11, the map $\widetilde{\sigma\left(\underline{z}_{k}\right)}$ lies in $\mathcal{P}^{*, k-1}$ as a linear combination of symbols $\tau_{j}\left(z_{1}, \ldots, z_{j}\right)$ in $\mathcal{P} \sum_{i=1}^{j} \alpha_{i}\left(z_{i}\right)+j-1, j-1, j$ varying from 1 to $k$.

By Proposition 9 the maps $\left(z_{1}, \ldots, z_{j}\right) \mapsto \sum \tau_{j}\left(z_{1}, \ldots, z_{j}\right)$ are meromorphic with poles of order $\leq j$ on a countable set of hypersurfaces $\sum_{i=1}^{j} \alpha_{i}\left(z_{i}\right) \in[-j, \infty[\cap \mathbb{Z}$. Consequently, the map $\left(z_{1}, \ldots, z_{l}\right) \mapsto \sum_{\leq}^{\text {Chen }} \sigma\left(\underline{z}_{k}\right)$ is meromorphic with poles of order $\leq j$ on the countable set of hypersurfaces $\sum_{i=1}^{j} \alpha_{i}\left(z_{i}\right) \in[-j, \infty[\cap \mathbb{Z}, j$ varying in $\{1, \ldots, k\}$.

2. Setting $z_{j}=z$ yields the second item of the proposition.

3. By Corollary 3 we know that under the assumptions of the theorem the nested sums converge. Consequently, the meromorphic map $\sum_{\leq}^{\text {Chen }} \sigma(z)$ is holomorphic at zero and the value at $z=0$ coincides with the ordinary nested sum. The stuffle relations then hold as a result of (45).

Applying this theorem to $\sigma_{i}\left(z_{i}\right)(\xi)=\chi(\xi) \xi^{-z_{i}}\left(\operatorname{resp} . \sigma_{i}\left(z_{i}\right)(\xi)=\left(\xi+v_{i}\right)^{-z_{i}}, v_{i}>0\right)$ leads to the following properties of multiple zeta functions. 
Corollary 4 The extensions of multiple (resp. Hurwitz) zeta functions $\zeta\left(z_{1}, \ldots, z_{k}\right)$ (resp. $\left.\zeta\left(z_{1}, \ldots, z_{k} ; v_{1}, \ldots, v_{k}\right)\right)$ given by Theorem 5 satisfy stuffle relations and are meromorphic in all variables with poles on a countable family of hyperplanes $z_{1}+\cdots+$ $\left.\left.z_{j} \in\right]-\infty, j\right] \cap \mathbb{Z}, j$ varying from 1 to $k$.

Remark 5 More can be said about the pole structure for multiple zeta functions of depth 2 as recalled in the introduction and as we shall see in Theorem 9.

When the nested sums do not converge, one does not expect the stuffle relations to hold in general neither for cut-off nested sums, nor for finite parts of meromorphic perturbations of these sums, because of extra terms which occur when taking finite parts that might spoil the stuffle relations. However, just as for nested integrals, one can use instead a renormalisation procedure which takes care of these extra terms and thereby build renormalised nested sums which do obey the required stuffle relations. For this purpose, we first extend a holomorphic regularisation $\mathcal{R}$ on $\mathcal{P}^{*, 0}$ to one on the tensor algebra $\mathcal{T}\left(\mathcal{P}^{*, 0}\right)$ which is compatible with the stuffle product (with respect to a product $\bullet$ which will be $\mp$ the ordinary product of functions). This can be carried out by twisting by the Hoffman isomorphism the previously extended regularisation $\tilde{\mathcal{R}}$ compatible with the shuffle product. Applying this isomorphism to any subalgebra $\mathcal{A}$ of $\mathcal{P}^{*, 0}$ provides a regularisation $\tilde{\mathcal{R}}^{\star}$ on $\mathcal{T}(\mathcal{A})$ compatible with the stuffle product $\star_{\bullet}$, just as $\tilde{\mathcal{R}}$ was with the shuffle product $\amalg$ (see (30) $)$ :

Lemma 4 A regularisation $\mathcal{R}$ on any subalgebra $\mathcal{A}$ of $\mathcal{P}^{*, 0}$ extends to one on $\mathcal{T}(\mathcal{A})$ defined by

$$
\tilde{\mathcal{R}}^{\star}:=\exp \circ \tilde{\mathcal{R}} \circ \log
$$

which is compatible with $\star_{\bullet}$ :

$$
\tilde{\mathcal{R}}^{\star}\left(\sigma \star_{\bullet} \tau\right)=\tilde{\mathcal{R}}^{\star}(\sigma) \star_{\bullet} \tilde{\mathcal{R}}^{\star}(\tau) \quad \forall \sigma, \tau \in \mathcal{T}\left(\mathcal{P}^{*, 0}\right) .
$$

Proof: This is an immediate consequence of Theorem 3 .

Proposition 12 : Let $W$ be an open neighbourhood of 0 in $\mathbb{C}$, let $\mathcal{A}$ be a subalgebra of $\mathcal{P}^{*, 0}$, and let $\mathcal{R}: \tau \mapsto\{\tau(z)\}_{z \in W}$ be a holomorphic regularisation on $\mathcal{A}$ such that the perturbed symbol $\sigma(z)$ of any symbol $\sigma$ has order $\alpha(z)$ with $\operatorname{Re} \alpha^{\prime}(z)<0$ for any $z \in W$. We suppose that for any real $t$ there is a $z \in W$ such that for any symbol $\sigma$ of real order $t$, the symbol $\sigma(z)$ has real order $<-1$. Then the map

$$
\begin{aligned}
\mathcal{A} & \rightarrow \mathcal{M}(W) \\
\sigma & \mapsto \sum \sigma(z)
\end{aligned}
$$

extends to multiplicative maps

$$
\begin{aligned}
\Psi^{\mathcal{R}}\left(\operatorname{resp} . \Psi^{\prime \mathcal{R}}\right):\left(\mathcal{T}(\mathcal{A}), \star_{\bullet}\right) & \rightarrow \mathcal{M}(W) \\
\sigma & \mapsto \sum_{<}^{\text {Chen }} \tilde{\mathcal{R}}^{\star}(\sigma)(z) \quad\left(\operatorname{resp} . \sum_{\leq}^{\text {Chen }} \tilde{\mathcal{R}}^{\star}(\sigma)(z)\right)
\end{aligned}
$$

where $\bullet$ stands for the ordinary product - in the first case, and stands for the opposite of the ordinary product in the second case. In other words, $\Psi^{\mathcal{R}}$ and $\Psi^{\prime \mathcal{R}}$ satisfy the stuffle relations:

$$
\begin{aligned}
\Psi^{\mathcal{R}}\left(\sigma \star_{+} \cdot \tau\right) & =\Psi^{\mathcal{R}}(\sigma) \cdot \Psi^{\mathcal{R}}(\tau), \\
\Psi^{\prime \mathcal{R}}\left(\sigma \star_{-} \cdot \tau\right) & =\Psi^{\prime \mathcal{R}}(\sigma) \cdot \Psi^{\prime \mathcal{R}}(\tau)
\end{aligned}
$$


which hold as equalities of meromorphic functions.

Proof: The proof is carried out along the same lines as for continuous integrals. By construction, $\tilde{\mathcal{R}}^{\star}\left(\sigma_{1} \otimes \cdots \otimes \sigma_{k}\right)(z)$ is a finite linear combination of bullet-products and tensor products of the $\mathcal{R}\left(\sigma_{i}\right)(z)$ 's. For example:

$$
\tilde{\mathcal{R}}^{\star}\left(\sigma_{1} \otimes \sigma_{2}\right)(z)=\sigma_{1}(z) \otimes \sigma_{2}(z)-\frac{1}{2}\left(\sigma_{1} \bullet \sigma_{2}\right)(z)+\frac{1}{2} \sigma_{1}(z) \bullet \sigma_{2}(z) .
$$

It therefore follows from Theorem 6 that the cut-off nested sums $\Psi^{\mathcal{R}}(\sigma)(z)$ and $\Psi^{\prime \mathcal{R}}(\sigma)(z)$ define meromorphic functions. Moreover, the compatibility of $\tilde{\mathcal{R}}^{\star}$ with the stuffle product $\star_{\bullet}$ implies that

$$
\begin{aligned}
\Psi^{\mathcal{R}}\left(\sigma \star_{\bullet} \tau\right)(z) & =\sum_{\text {Chen }}^{\text {Chen }} \tilde{\mathcal{R}}^{\star}\left(\sigma \star_{\bullet} \tau\right)(z) \\
& =\sum_{\text {Chen }}^{\star} \tilde{\mathcal{R}}^{\star}(\sigma)(z) \star_{\bullet} \tilde{\mathcal{R}}^{\star}(\tau)(z) \\
& =\sum^{\star} \tilde{\mathcal{R}}^{\star}(\sigma)(z) \cdot \sum^{\text {Chen }} \tilde{\mathcal{R}}^{\star}(\tau)(z), \\
& =\Psi^{\mathcal{R}}(\sigma)(z) \star_{\bullet} \Psi^{\mathcal{R}}(\tau)(z),
\end{aligned}
$$

holds on a common domain of convergence of these sums as a result of the usual stuffle relations for convergent nested sums (see (52)). This identity of holomorphic functions on some common domain (which is non-empty due to the hypothesis on $W$ and the regularisation procedure) then extends by analytic continuation to an identity of meromorphic functions.

Birkhoff factorisation provides a way to extract finite parts which still obey stuffle relations:

Theorem 7 Given a subalgebra $\mathcal{A} \subset \mathcal{P}^{*, 0}$ and a holomorphic regularisation $\mathcal{R}: \sigma \mapsto$ $\{\sigma(z)\}_{z \in W}$ on $\mathcal{A}$ which satisfies the same assumption as in Proposition 12, the map

$$
\begin{aligned}
\mathcal{A} & \rightarrow \mathbb{C} \\
\sigma & \mapsto \mathrm{fp}_{z=0} \sum \sigma(z)
\end{aligned}
$$

extends to a character:

$$
\begin{aligned}
\psi^{\mathcal{R}}\left(\text { resp. } \psi^{\prime \mathcal{R}}\right):\left(\mathcal{T}(\mathcal{A}), \star_{\bullet}\right), & \longrightarrow \mathbb{C} \\
\sigma & \mapsto \Psi_{+}^{\mathcal{R}}(\sigma)(0) \quad\left(\text { resp. } \Psi_{+}^{\prime \mathcal{R}}(\sigma)(0)\right)
\end{aligned}
$$

defined from the Birkhoff decomposition of $\Psi^{\mathcal{R}}$ (resp. $\left.\Psi^{\prime \mathcal{R}}\right)$. It coincides with the ordinary nested sums $\sum_{<}^{\text {Chen }}$ (resp. $\sum_{\leq}^{\text {Chen }}$ ) on elements $\sigma=\sigma_{1} \otimes \cdots \otimes \sigma_{k}$ with $\sigma_{j} \in \mathcal{P}^{\alpha_{j}, 0}$ when $\operatorname{Re}\left(\alpha_{1}+\cdots+\alpha_{m}\right)<-m$ for any $m \in\{1, \ldots, k\}$. Here $\bullet$ stands for the product \pm . as in Proposition 12 .

Proof: Let us prove the strict inequality case: the Birkhoff decomposition [CK], $\mathrm{M}$ ] for the minimal subtraction scheme reads:

$$
\Psi^{\mathcal{R}}=\left(\Psi_{-}^{\mathcal{R}}\right)^{*-1} * \Psi_{+}^{\mathcal{R}}
$$


with $\bullet=$ ( see Theorem 2 2). Since $\Psi_{+}^{\mathcal{R}}$ is multiplicative on $\left(\mathcal{T}(\mathcal{A}), \star_{\bullet}\right)$, it obeys the stuffle relation:

$$
\Psi_{+}^{\mathcal{R}}\left(\sigma \star_{\bullet} \tau\right)(z)=\Psi_{+}^{\mathcal{R}}(\sigma)(z) \Psi_{+}^{\mathcal{R}}(\tau)(z)
$$

which holds as an equality of meromorphic functions holomorphic at $z=0$. Setting

$$
\psi^{\mathcal{R}}:=\Psi_{+}^{\mathcal{R}}(0)
$$

and applying the stuffle relations (56) at $z=0$ yields

$$
\psi^{\mathcal{R}}\left(\sigma \star_{\bullet} \tau\right)=\psi^{\mathcal{R}}(\sigma) \psi^{\mathcal{R}}(\tau)
$$

The tensor products $\sigma=\sigma_{1} \otimes \cdots \otimes \sigma_{k}$ with $\sigma_{j} \in \mathcal{P}^{\alpha_{j}, 0}$ where $\operatorname{Re}\left(\alpha_{1}+\cdots+\alpha_{m}\right)<-m$ for any $m \in\{1, \ldots, k\}$ span a right co-ideal $\mathcal{J}$ of $\mathcal{T}(\mathcal{A})$, namely $\Delta(\mathcal{J}) \subset \mathcal{J} \otimes \mathcal{T}(\mathcal{A})$. The restriction of $\Psi^{\mathcal{R}}$ to this right co-ideal takes values into functions which are holomorphic at $z=0$. By construction we then have:

$$
\psi^{\mathcal{R}}(\sigma)=\Psi_{+}^{\mathcal{R}}(\sigma)(0)=\Psi^{\mathcal{R}}(\sigma)(0)=\sum_{\leq}^{\text {Chen }} \sigma
$$

by a similar argument as in the proof of Theorem 2 The weak inequality case can be derived similarly setting $\bullet=-\cdot$.

On the grounds of this result we set the following definition:

Definition 10 For any $\sigma \in \mathcal{T}(\mathcal{A})$, the renormalised nested sums of $\sigma$ (in both weak and strict inequality versions) are defined by:

$$
\sum_{<}^{\text {Chen, } \mathcal{R}} \sigma:=\psi^{\mathcal{R}}(\sigma), \quad \sum_{\leq}^{\text {Chen, } \mathcal{R}} \sigma:=\psi^{\prime \mathcal{R}}(\sigma) .
$$

Remark 6 There are other multiplicative maps from $\left(\mathcal{T}(\mathcal{A}), \star_{\bullet}\right)$ to $\mathbb{C}$ defined by $\phi_{r}^{\mathcal{R}}$ 。 $\log$ with the notations of Theorem 2 . Those maps are not related to nested sums. 


\section{RENORMALISED MULTIPLE ZETA FUNCTIONS}

Recall that multiple Hurwitz zeta functions $\zeta\left(s_{1}, \ldots, s_{k} ; v_{1}, \ldots, v_{k}\right)$ and $\bar{\zeta}\left(s_{1}, \ldots, s_{k} ; v_{1}, \ldots, v_{k}\right)$ converge whenever $\operatorname{Re}\left(s_{1}+\cdots+s_{m}\right)>m$ for any $m \in\{1, \ldots, k\}$, in which case they obey stuffle relations. In this section, we implement the renormalisation procedure described in section 10 to extend them to other complex values of $s_{i}$ while preserving the stuffle relations. We then specialise to nonnegative integer arguments for which we prove rationality of the renormalised multiple zeta values.

\subsection{Stuffle relations for renormalised multiple zeta functions}

Let $\widetilde{\mathcal{A}}$ be the subalgebra of $\mathcal{P}^{*, 0}$ generated by the continuous functions with support inside the interval $] 0,1[$ and the set

$$
\left\{f \in \mathcal{P}^{*, 0}, \exists v \in \mathbb{R}_{+}, \exists s \in \mathbb{C}, f(\xi)=(\xi+v)^{-s} \text { when } \xi \geq 1\right\} .
$$

Consider the ideal $\mathcal{N}$ of $\widetilde{\mathcal{A}}$ of continuous functions with support included in the interval ]0,1[. The quotient algebra $\mathcal{A}=\widetilde{\mathcal{A}} / \mathcal{N}$ is then generated by the elements $\sigma_{s}^{v}$, where $\sigma_{s}^{v}$ is the class of any $f \in \widetilde{\mathcal{A}}$ such that $f(\xi)=(\xi+v)^{-s}$ for $\xi \geq 1$. Notice that for any $v \in \mathbb{R}_{+}$the subspace $\mathcal{A}_{v}$ of $\mathcal{A}$ generated by $\left\{\sigma_{s}^{v}, s \in \mathbb{C}\right\}$ is a subalgebra of $\mathcal{A}$. We choose the product $\bullet$ as the opposite of the ordinary product, so that we have:

$$
\sigma \bullet \sigma^{\prime}=-\sigma \sigma^{\prime} \quad \forall\left(\sigma, \sigma^{\prime}\right) \in \mathcal{A}^{2} ; \quad \text { resp. } \quad \sigma_{s}^{v} \bullet \sigma_{s^{\prime}}^{v}=-\sigma_{s+s^{\prime}}^{v} \quad \forall\left(\sigma_{s}^{v}, \sigma_{s^{\prime}}^{v}\right) \in \mathcal{A}_{v}^{2} .
$$

Let $\mathcal{W}$ be the $\mathbb{C}$-vector space freely spanned by sequences $\left(u_{1}, \ldots, u_{k}\right)$ of real numbers. Let us define the stuffle product on $\mathcal{W}$ by:

$$
\left(u_{1}, \ldots, u_{k}\right) \star\left(u_{k+1}, \ldots, u_{k+l}\right)=\sum_{0 \leq r \leq \min (k, l)}(-1)^{r} \sum_{\pi \in \operatorname{mix} \operatorname{sh}(k, l ; r)}\left(u_{1}^{\pi}, \ldots, u_{k+l-r}^{\pi}\right),
$$

with:

$$
u_{j}^{\pi}=\sum_{i \in\{1, \ldots, k+l\}, \pi(i)=j} u_{i}
$$

(the sum above contains only one or two terms). Define a map $u \mapsto \sigma_{u}^{v}$ from $W$ to $\mathcal{T}\left(\mathcal{A}_{v}\right)$ by:

Then

$$
\sigma_{\left(u_{1}, \ldots, u_{k}\right)}^{v}:=\sigma_{u_{1}}^{v} \otimes \cdots \otimes \sigma_{u_{k}}^{v} .
$$

$$
\sigma_{u}^{v} \star_{\bullet} \sigma_{u^{\prime}}^{v}=\sigma_{u \star u^{\prime}}^{v}
$$

The same holds with $\bullet=\cdot$ provided we drop the signs $(-1)^{r}$ in equation (57) defining the stuffle product on $\mathcal{W}$.

Definition 11 Let $W$ be an open neighbourhood of 0 in $\mathbb{C}$. Let $\mathcal{R}: \sigma \mapsto\{\sigma(z)\}_{z \in W}$ be a holomorphic regularisation procedure on $\widetilde{\mathcal{A}}$ such that the order condition of Proposition 12 is satisfied, and which respects the ideal $\mathcal{N}$, hence giving rise to a regularisation procedure on the quotient $\mathcal{A}$. The renormalised multiple Hurwitz zeta functions (with respect to $\mathcal{R}$ ) are defined by:

$$
\begin{aligned}
& \zeta^{\mathcal{R}}\left(s_{1}, \ldots, s_{k} ; v_{1}, \ldots, v_{k}\right):=\psi^{\mathcal{R}}\left(\sigma_{s_{1}}^{v_{1}} \otimes \cdots \otimes \sigma_{s_{k}}^{v_{k}}\right), \\
& \bar{\zeta}^{\mathcal{R}}\left(s_{1}, \ldots, s_{k} ; v_{1}, \ldots, v_{k}\right):=\psi^{\prime \mathcal{R}}\left(\sigma_{s_{1}}^{v_{1}} \otimes \cdots \otimes \sigma_{s_{k}}^{v_{k}}\right) .
\end{aligned}
$$

For $v_{1}=\cdots=v_{k}=v$ we simply set

$$
\zeta^{\mathcal{R}}\left(s_{1}, \ldots, s_{k} ; v\right):=\psi^{\mathcal{R}}\left(\sigma_{s_{1}, \ldots, s_{k}}^{v}\right) ; \quad \bar{\zeta}^{\mathcal{R}}\left(s_{1}, \ldots, s_{k} ; v\right):=\psi^{\prime \mathcal{R}}\left(\sigma_{s_{1}, \ldots, s_{k}}^{v}\right) .
$$


By Theorem 7 applied to $\widetilde{\mathcal{A}}$ defined at the beginning of the section, combined with the fact that the nested sums and the regularisation are well-defined on the quotient $\mathcal{A}$, we know that $\psi^{\mathcal{R}}$ is compatible with the stuffle product:

$$
\psi^{\mathcal{R}}\left(\left(\sigma_{s_{1}}^{v_{1}} \otimes \cdots \otimes \sigma_{s_{k}}^{v_{k}}\right) \star\left(\sigma_{s_{1}^{\prime}}^{v_{1}^{\prime}} \otimes \cdots \otimes \sigma_{s_{k^{\prime}}^{\prime}}^{v_{k^{\prime}}^{\prime}}\right)\right)=\psi^{\mathcal{R}}\left(\sigma_{s_{1}}^{v_{1}} \otimes \cdots \otimes \sigma_{s_{k}}^{v_{k}}\right) \psi^{\mathcal{R}}\left(\sigma_{s_{1}^{\prime}}^{v_{1}^{\prime}} \otimes \cdots \otimes \sigma_{s_{k^{\prime}}^{\prime}}^{v_{k^{\prime}}^{\prime}}\right)
$$

and similarly for $\psi^{\prime \mathcal{R}}$. When setting the $v_{i}$ 's equal to some fixed $v$, Theorem 7 applied to $\widetilde{\mathcal{A}}_{v}$ leads to the following stuffle properties of families of Hurwitz multiple zeta functions which contain the ordinary multiple zeta functions obtained by setting $v=0$.

Theorem 8 1. Renormalised Hurwitz multiple zeta values have the following properties:

$$
\bar{\zeta}^{\mathcal{R}}\left(u \star u^{\prime} ; v\right)=\bar{\zeta}^{\mathcal{R}}(u ; v) \bar{\zeta}^{\mathcal{R}}\left(u^{\prime} ; v\right)
$$

when the stuffle product $\star$ is defined by (57), and:

$$
\zeta^{\mathcal{R}}\left(u \star u^{\prime} ; v\right)=\zeta^{\mathcal{R}}(u ; v) \zeta^{\mathcal{R}}\left(u^{\prime} ; v\right)
$$

when the stuffle product $\star$ is defined by $\sqrt{57})$ with signs $(-1)^{r}$ removed.

2. for any positive integer $k$, and for $\left(s_{1}, \ldots, s_{k}\right) \in \mathbb{C}^{k}$ such that $\operatorname{Re}\left(s_{1}+\cdots+s_{m}\right)>$ $m$ for any $m \in\{1, \ldots, k\}$, the renormalised values are independent of $\mathcal{R}$ and can be written as ordinary nested sums:

$$
\begin{aligned}
\bar{\zeta}^{\mathcal{R}}\left(s_{1}, \ldots, s_{k} ; v\right) & =\sum_{0<s_{1} \leq \cdots \leq n_{k}}\left(n_{1}+v\right)^{-s_{1}} \cdots\left(n_{k}+v\right)^{-s_{k}}=\bar{\zeta}\left(s_{1}, \ldots, s_{k} ; v\right), \\
\zeta^{\mathcal{R}}\left(s_{1}, \ldots, s_{k} ; v\right) & =\sum_{0<s_{1}<\cdots<n_{k}}\left(n_{1}+v\right)^{-s_{1}} \cdots\left(n_{k}+v\right)^{-s_{k}}=\zeta\left(s_{1}, \ldots, s_{k} ; v\right) .
\end{aligned}
$$

This provides the uniqueness of the extension of Riemann multiple zeta functions to regularised multiple zeta functions satisfying stuffle relations, once the value $\theta$ at the argument 1 is imposed; see [H], W], ZZ]. Indeed, the expressions $\bar{\zeta}^{\mathcal{R}}\left(s_{1}, \ldots, s_{k}\right)$ and $\zeta^{\mathcal{R}}\left(s_{1}, \ldots, s_{k}\right)$ converge whenever $s_{1}>1$ since by assumption, all the $s_{i}$ are no smaller than 1. When they converge, they obey the stuffle relations (58) and (59) respectively. The uniqueness of the extension to the case $s_{1}=1$ then follows by induction on the length $k$ from the stuffle relations (58) which "push" the leading term $s_{1}=1$ whenever it arises, away from the first position and therefore expresses divergent expressions in terms of convergent expressions. Given a real number $\mu$, we can consider the holomorphic regularisation (cfr. (34))

$$
\xi \mapsto \mathcal{R}_{\mu}(\tau)(\xi):=\tau(\xi)|\xi|^{-\gamma_{\mu}(z)}
$$

which is well defined on $\mathcal{A}$. For example we can consider $\gamma_{\mu}(z)=z+\mu z^{2}$. In this case the parameter $\mu$ plays the role of the constant $\theta$.

\subsection{Rationality of (Hurwitz) multiple zeta values at nonposi- tive arguments}

The following theorem provides refined meromorphicity and holomorphicity results for multiple zeta functions which go beyond the meromorphicity results of Corollary 4. 
Theorem 9 Let $\gamma$ be a holomorphic function in an open neighbourhood $W$ on 0 in $\mathbb{C}$ such that $\gamma(0)=0$ and $\gamma^{\prime}(0)=1$. Let $l \in \mathbb{N}-\{0\}$. Let $\left(\beta_{1}, \ldots, \beta_{l}\right)$ be functions $\beta_{j}(z)=b_{j}-c_{j} \gamma(z)$ such that $\operatorname{Re} c_{j}>0$ for $j=1, \ldots l$.

1. For any $v_{1} \geq 0, \ldots, v_{l} \geq 0$ the maps

$$
\left(z_{1}, \ldots, z_{l}\right) \longmapsto \sum_{1 \leq n_{l}<\cdots<n_{1}}\left(n_{1}+v_{1}\right)^{\beta_{1}\left(z_{1}\right)} \cdots\left(n_{l}+v_{l}\right)^{\beta_{l}\left(z_{l}\right)}
$$

are meromorphic with poles on hyperplanes $\sum_{i=1}^{m} c_{i} z_{i} \in-\sum_{i=1}^{m} b_{i}-m+\mathbb{N}, m$ varying in $\{1, \ldots, l\}$ so that poles at $\left(z_{1}, \ldots, z_{l}\right)=(0, \ldots, 0)$ can arise whenever $\sum_{i=1}^{m} b_{i} \in\left[-m ;+\infty\left[\cap \mathbb{Z}\right.\right.$. When $l=2$ and $v_{1}=\cdots=v_{l}=v$ for some non negative real number $v$, poles actually only arise when $b_{1}=-1$ or $b_{1}+b_{2} \in$ $\{-2,-1,0,2,4,6, \ldots\}$.

2. Let us assume that $b_{1}, \ldots, b_{l-1} \in \mathbb{N}$ and $b_{l} \in \mathbb{Z}$.

- If $b_{l} \in \mathbb{N}$, for any non negative real number $v$, the map

$$
z \longmapsto \sum_{1 \leq n_{l}<\cdots<n_{1}}\left(n_{1}+v\right)^{\beta_{1}(z)} \cdots\left(n_{l}+v\right)^{\beta_{l}(z)}
$$

is holomorphic around $z=0$. Its value at $z=0$ is a polynomial expression in $v$ with coefficients given by rational functions in the $c_{j}$ 's that do not depend on the choice of $\gamma$.

- If $b_{l} \leq-1$, the above map is meromorphic with a possible pole of order at most 1 at $z=0$. The residue at $z=0$ is a polynomial expression in $v$ with coefficients given by rational functions in the $c_{j}$ 's that do not depend on the choice of $\gamma$.

Remark 7 Our proof, which relies on the algebra structure of the set $\{\xi \mapsto(\xi+$ $\left.v)^{a}, \quad \xi>0, a \in \mathbb{N}\right\}$ does not a priori extend to show holomorphicity and rationality for all non negative values $v_{1}, \ldots, v_{k}$ which therefore remains an open question.

Proof: By Theorem 6 applied to $\sigma_{i}(\xi)=\left(\xi+v_{i}\right)^{-s_{i}} \chi(x)$ where $\chi$ is a smooth cut-off function which vanishes at zero and is identically one outside the unit interval, the maps

$$
\left(z_{1}, \ldots, z_{l}\right) \longmapsto \sum_{1 \leq n_{l}<\cdots<n_{1}}\left(n_{1}+v_{1}\right)^{\beta_{1}\left(z_{1}\right)} \cdots\left(n_{l}+v_{l}\right)^{\beta_{l}\left(z_{l}\right)}
$$

are meromorphic with poles on hyperplanes $\sum_{i=1}^{m} c_{i} z_{i} \in-\sum_{i=1}^{m} b_{i}-m+\mathbb{N}$ with $m$ varying in $\{1, \ldots, l\}$ from which we infer the first part of the proposition concerning the pole structure for a general $l$ and general $b_{i}$ 's which generalise the results of Corollary 4. In the case $l=2$, more can be said on the structure of the poles; we postpone the proof, leaving this case for later. Since $\operatorname{Re}\left(c_{i}\right)<0$ for any $i \in\{1, \ldots, l\}$ we can set $z_{i}=z$, from which the meromorphicity of the map $z \mapsto \sum_{1 \leq n_{l}<\cdots<n_{1}}\left(n_{1}+\right.$ $\left.v_{1}\right)^{\beta_{1}(z)} \cdots\left(n_{l}+v_{l}\right)^{\beta_{l}(z)}$ follows. We actually recover the meromorphicity of this map by the inductive proof below. To prove Part 2 of the theorem, we indeed proceed by induction on the depth $l$ and set in order to lighten the notations:

$$
\sigma_{j}(z)(\xi):=(\xi+v)^{\beta_{j}(z)}, \quad \text { with } \beta_{j}(z)=b_{j}-c_{j} \gamma(z),
$$

for $\xi \geq 1$. 
1. In the case $l=1$ we have $\sum_{1 \leq n_{1}}\left(n_{1}+v\right)^{\beta_{1}(z)}=\zeta\left(-\beta_{1}(z) ; v\right)$ where, for any $v \geq 0$, we have set $\zeta(s ; v)=\sum_{n=1}^{\infty}(n+v)^{-s}$ which relates to the usual Hurwitz zeta function $\zeta(s, v):=\sum_{n=0}^{\infty}(n+v)^{-s}$ by $\zeta(s ; v)=\zeta(s, v+1)$. The first step of the induction follows from Proposition 10 which gives

$$
\operatorname{Res}_{z=0} \zeta\left(-\beta_{1}(z)\right)=\delta_{b_{1}+1} \frac{1}{c_{1}} ; \quad \zeta(-k ; v)=-\frac{B_{k+1}(1+v)}{k+1} \quad \text { if } \quad b_{1} \in \mathbb{N} .
$$

2. Now suppose $l \geq 2$. It is useful to observe that each summation raises the order of the corresponding interpolated symbols by 1 , so that to pick out the finite part as $N \rightarrow \infty$, which amounts to extracting the homogeneous part of the symbol of degree 0 , we need to take into consideration homogeneous parts of the symbol of negative degree as we go into the depths of the nested sums, i.e. of degree $\geq-J$ after $J$ summations. For a symbol $\sigma$ of order $a$ and for any real $\omega$ we call $\sigma_{\geq \omega}$ the (finite) sum of terms of real order $\geq \omega$ in the asymptotic expansion of the symbol $\sigma$. We have for any $z$ in a small neighbourhood of 0 :

$$
\begin{gathered}
\left(\sum_{0<n_{l}<\cdots<n_{1}<N}\left(n_{1}+v\right)^{\beta_{1}(z)} \cdots\left(n_{l}+v\right)^{\beta_{l}(z)}\right)_{\geq 0} \\
=\left(\sum_{n_{1}=1}^{N-1}\left(n_{1}+v\right)^{\beta_{1}(z)}\left(\sum_{0<n_{l}<\cdots<n_{1}}\left(n_{2}+v\right)^{\beta_{2}(z)} \cdots\left(n_{l}+v\right)^{\beta_{l}(z)}\right)_{\geq-b_{1}-1}\right)_{\geq 0} \\
=\left(\sum_{n_{1}=0}^{N-1}\left(n_{1}+v\right)^{\beta_{1}(z)}\left(\sum_{n_{2}=1}^{n_{1}-1}\left(n_{2}+v\right)^{\beta_{2}(z)} \ldots\left(\sum_{n_{l}=1}^{n_{l-1}-1}\left(n_{l}+v\right)^{\beta_{l}(z)}\right)_{\geq-b_{l-1}-b_{l-2} \cdots-b_{1}-l+1} \ldots\right)_{\geq-b_{1}-1}\right)_{\geq 0} .
\end{gathered}
$$

3. We extend the notation $[a]_{j}$ to $j=0$ and $j=-1$ by setting:

$$
[a]_{0}:=1, \quad[a]_{-1}:=\frac{1}{a+1} .
$$

Let us use (42) to expand the interpolating symbol $\bar{Q}\left(\sigma_{l}(z)\right)$ for the deepest sum $Q\left(\sigma_{l}(z)\right)(N)=\sum_{0<n_{l}<N} \sigma_{l}(z)\left(n_{l}\right)$, writing:

$$
\begin{aligned}
\bar{Q}\left(\sigma_{l}(z)\right)(\xi)= & \sum_{j=0}^{2 J_{l}} B_{j} \frac{\left[\beta_{l}(z)\right]_{j-1}}{j !}\left((\xi+v)^{\beta_{l}(z)-j+1}-1\right) \\
& +\frac{\left[\beta_{l}(z)\right]_{2 J_{l}+1}}{\left(2 J_{l}+1\right) !} \int_{1}^{\xi} \overline{B_{2 J_{l}+1}}(y)(y+v)^{\beta_{l}(z)-2 J_{l}-1} d y
\end{aligned}
$$

choosing $J_{l} \geq\left[\frac{1}{2}\left(\sum_{j=1}^{l} b_{j}+l\right)\right]$ we have $b_{l}-2 J_{l}-1<-b_{1}-\cdots-b_{l-1}-l+1$ so that we do not miss any terms in (63).

4. The $l=2$ case is instructive and worth being treated separately since it presents some specificities; in that case $J_{2} \geq\left[\frac{1}{2}\left(b_{1}+b_{2}\right)\right]+1$ so that if $b_{1}+b_{2}$ is even, we take $J_{2}=\frac{1}{2}\left(b_{1}+b_{2}\right)+1$ and if $b_{1}+b_{2}$ is odd, we take $J_{2}=\frac{1}{2}\left(b_{1}+b_{2}\right)+\frac{3}{2}$. 
By (65), we have

$$
\begin{aligned}
\sum_{<}^{\text {Chen }} \sigma_{1}\left(z_{1}\right) \otimes \sigma_{2}\left(z_{2}\right)= & \sum_{0}^{\infty} \sigma_{1}\left(z_{1}\right) \bar{Q}\left(\sigma_{2}\left(z_{2}\right)\right) \\
= & \sum_{j=0}^{2 J_{2}} B_{j} \frac{\left[\beta_{2}\left(z_{2}\right)\right]_{j-1}}{j !}\left(\sum_{0}^{\infty}(n+v)^{\beta_{1}\left(z_{1}\right)}(n+v)^{\beta_{2}\left(z_{2}\right)-j+1}-\sum_{0}^{\infty}(n+v)^{\beta_{1}\left(z_{1}\right)}\right) \\
& -\frac{\left[\beta_{2}\left(z_{2}\right)\right]_{2 J_{2}+1}}{\left(2 J_{2}+1\right) !} \sum_{0}^{\infty}\left((n+v)^{\beta_{1}\left(z_{1}\right)} \int_{1}^{n} \overline{B_{2 J_{l}+1}}(y)(y+v)^{\beta_{2}\left(z_{2}\right)-2 J_{2}-1} d y\right) . \\
= & \sum_{j=0}^{2 J_{2}} B_{j} \frac{\left[\beta_{2}\left(z_{2}\right)\right]_{j-1}}{j !}\left(\zeta\left(-\beta_{1}\left(z_{1}\right)-\beta_{2}\left(z_{2}\right)+j-1 ; v\right)-\zeta\left(-\beta_{1}\left(z_{1}\right) ; v\right)\right. \\
& +\frac{\left[\beta_{2}\left(z_{2}\right)\right]_{2 J_{2}+1}}{\left(2 J_{2}+1\right) !} \sum_{0}^{\infty}\left((n+v)^{\beta_{1}\left(z_{1}\right)} \int_{1}^{n} \overline{B_{2 J_{l}+1}}(y)(y+v)^{\beta_{2}\left(z_{2}\right)-2 J_{2}-1} d y\right)
\end{aligned}
$$

Since $J_{2} \geq \frac{1}{2}\left(b_{1}+b_{2}\right)+1$, it follows that $b_{1}+b_{2}-2 J_{2}<-1$ so that the last term is absolutely convergent and hence holomorphic. Moreover, we observe that $\left[b_{2}\right]_{2 J_{2}+1}=0$ whenever $b_{2}$ is an integer since $b_{2}-2 J_{2} \leq-b_{1}-2<0$, so that the last term actually vanishes at $\left(z_{1}, z_{2}\right)=(0,0)$ in that case. The map $\left(z_{1}, z_{2}\right) \mapsto \sum_{<}^{\text {Chen }} \sigma_{1}\left(z_{1}\right) \otimes \sigma_{2}\left(z_{2}\right)$ is therefore meromorphic with poles on a finite number of hyperplanes $b_{1}-c_{1} z_{1}=-1$ and $c_{1} z_{1}+c_{2} z_{2}=b_{1}+b_{2}-j+2, \quad j \in$ $\left\{0,1,2,4, \ldots, 2 J_{2}\right\}$ since Bernoulli numbers $B_{j}$ vanish for odd $j$ larger than 2 and the only pole of the zeta function is 1 . Poles at zero therefore only arise if $b_{1}=-1$ or $b_{1}+b_{2} \in\{-2,-1,0,2,4,6, \ldots\}$, thus confirming known results AET, see also Zh and G] Theorem 2.25.

The map is actually holomorphic at zero for any value of $\left(b_{1}, b_{2}\right)$ along any hyperplane $z_{1}=\lambda z_{2}$ with $\lambda>0$. Indeed, setting $z_{1}=\lambda z_{2}$ in the above expression gives rise to (simple) poles in $z$ which are compensated by terms $\left[\beta_{2}(z)\right]_{j-1}$ since these involve a factor $z$ as a consquence of the inequality $b_{2}-j+$ $2=-b_{1} \leq 0$ resulting from $b_{1}+b_{2}+2=j$. Combining the above results moreover shows that the evaluation at $z=0$ gives rise to a rational number whenever $b_{1}$ and $b_{2}$ are integers as a result of the rationality of Bernoulli numbers.

5. Let us now assume $l \geq 2$, thus including the case $l=2$ even though it was treated separately. We write:

$$
\sum_{<}^{\text {Chen }} \sigma_{1}(z) \otimes \cdots \otimes \sigma_{l}(z)=\sum \sigma_{1}(z) \otimes \cdots \otimes \sigma_{l-2}\left(z_{l-2}\right) \otimes \sigma_{l-1}\left(z_{l-1}\right) \bar{Q}\left(\sigma_{l}\right)\left(z_{l}\right)
$$

which is a finite linear combination of nested sums $\sum_{<}^{\text {Chen }} \sigma_{1}(z) \otimes \cdots \otimes \sigma_{l-2}(z) \otimes$ $\sigma_{l-1}(z) \sigma_{l, j}(z)$ of depth $l-1$ : with the above choice of $J_{l}, b_{l}-2 J_{l} \leq-\sum_{i=1}^{l} b_{i}-$ $l+1<0$ so that by similar argument as in the $l=2$ case, one checks that the remainder term $\sigma_{1}(z) \otimes \cdots \otimes \sigma_{l-2}(z) \otimes \sigma_{l-1}(z) \rho_{\beta_{l}}(z)$ with:

$$
\rho_{\beta_{l}}(z)(\xi):=-\frac{\left[\beta_{l}\left(z_{l}\right)\right]_{2 J_{l}+1}}{\left(2 J_{l}+1\right) !} \int_{1}^{\xi} \overline{B_{2 J_{l}+1}}(y)(y+v)^{\beta_{l}\left(z_{l}\right)-2 J_{l}-1} d y
$$


contributes by a holomorphic term which vanishes at $z=0$. Applying (65) we have:

$$
\begin{aligned}
& \sum_{1 \leq n_{l}<\cdots<n_{l}}\left(n_{1}+v\right)^{\beta_{1}(z)} \cdots\left(n_{l}+v\right)^{\beta_{l}(z)} \\
& =\sum_{j=0}^{2 J_{l}} H_{l}^{j}\left(z_{l}\right) \sum_{1 \leq n_{l-1}<\cdots<n_{1}}\left(n_{1}+v\right)^{\beta_{1}\left(z_{1}\right)} \cdots\left(n_{l-2}+v\right)^{\beta_{l-2}(z)}\left(n_{l-1}+v\right)^{\kappa_{l-1}^{j}\left(z_{l-1}, z_{l}\right)}
\end{aligned}
$$

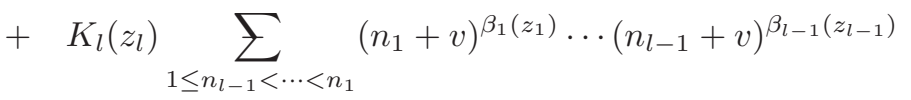

with

$$
\begin{gathered}
\kappa_{l-1}^{j}\left(z_{l-1}, z_{l}\right)=\beta_{l-1}\left(z_{l-1}\right)+\beta_{l}\left(z_{l}\right)+1-j, \\
H_{l}^{j}\left(z_{l}\right)=\frac{B_{j}}{j !}\left[\beta_{l}\left(z_{l}\right)\right]_{j-1}, \\
K_{l}\left(z_{l}\right)=-\sum_{j=0}^{2 J_{l}} \frac{B_{j}}{j !}\left[\beta_{l}\left(z_{l}\right)\right]_{j-1}+\rho_{\beta_{l}\left(z_{l}\right)} .
\end{gathered}
$$

The function $z \mapsto K_{l}(z)$ is holomorphic at $z=0$ unless $b_{l}=-1$. The functions $z \mapsto H_{l}^{j}(z)$ are holomorphic in $z=0$ unless $j=0$ and $b_{l}=-1$. If $b_{1}=-1$, the functions $z \mapsto H_{l}^{0}(z)$ and $z \mapsto K_{l}(z)$ have a simple pole in $z=0$ with residue $-\frac{1}{c_{l}}$ and $\frac{1}{c_{l}}$ respectively.

6. We are now ready to carry out the proof of the second item in the proposition by induction on the depth $l$. Suppose that the proposition is verified up to depth $l-1$. The left-hand side of (66) is then meromorphic at $z=0$. For any meromorphic function $f$ let us call $\operatorname{Res}_{z=0}^{j}(f)$ the coefficient of $z^{-j}$ in the Laurent expansion of $f$ at $z=0$. In particular $\operatorname{Res}_{z=0}^{0}(f)$ stands for the finite part, $\operatorname{Res}_{z=0}^{1}(f)$ stands for the ordinary residue and $\operatorname{Res}_{z=0}^{-1}(f)$ stands for the coefficient of $z$ in the Laurent expansion of $f(z)$. Picking out the coefficient of $z^{-m}$ in (66) (for some $m \in \mathbb{Z}$ ) we get:

$$
\begin{aligned}
& \sum_{1 \leq n_{l}<\cdots<n_{1}}\left(n_{1}+v\right)^{\beta_{1}(z)} \cdots\left(n_{l}+v\right)^{\beta_{l}(z)}
\end{aligned}
$$

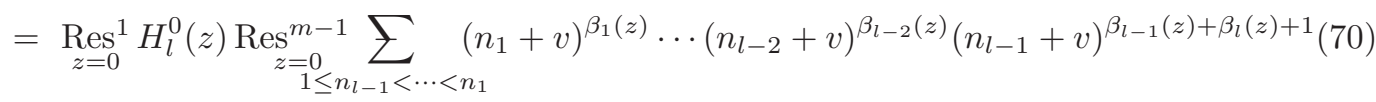

$$
\begin{aligned}
& +\operatorname{Res}_{z=0}^{1} K_{l}(z) \operatorname{Res}_{z=0}^{m-1} \sum_{1 \leq n_{l-1}<\cdots<n_{1}}\left(n_{1}+v\right)^{\beta_{1}(z)} \cdots\left(n_{l-1}+v\right)^{\beta_{l-1}(z)}
\end{aligned}
$$

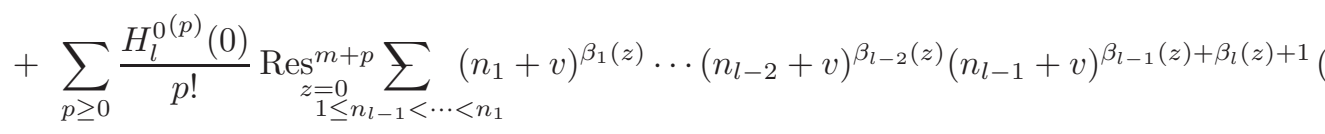

$$
\begin{aligned}
& +\sum_{p \geq 0} \frac{K_{l}^{(p)}(0)}{p !} \operatorname{Res}_{z=0}^{m+p} \sum_{1 \leq n_{l-1}<\cdots<n_{1}}\left(n_{1}+v\right)^{\beta_{1}(z)} \cdots\left(n_{l-1}+v\right)^{\beta_{l-1}(z)} \\
& +\sum_{j=1}^{2 J_{l}} \sum_{p \geq 0} \frac{H_{l}^{j(p)}(0)}{p !} \operatorname{Res}_{z=0}^{m+p} \sum_{1 \leq n_{l-1}<\cdots<n_{1}}\left(n_{1}+v\right)^{\beta_{1}(z)} \cdots\left(n_{l-2}+v\right)^{\beta_{l-2}(z)}\left(n_{l-1}+v\right)^{\kappa_{l-1}^{j}(z)} \text {. }
\end{aligned}
$$


- Suppose first $b_{l} \in \mathbb{N}$ and $m \geq 1$ in the equation above. The terms (70) and (71) vanish. The terms (72) and (73) also vanish according to the induction hypothesis. According to the induction assumption, all the terms in the sum (74) vanish as well, except possibly when $p=0$ and $m=1$, and when $j$ is big enough to ensure $\kappa_{l-1}^{j}(0) \leq-1$. But in that case $j \geq b_{l-1}+b_{l}+2 \geq b_{l}+2$, which implies $H_{l}^{j}(0)=0$. The left-hand side vanishes then for any $m \geq 1$, hence the function $z \mapsto \sum_{1 \leq n_{l}<\cdots<n_{1}}\left(n_{1}+v\right)^{\beta_{1}(z)} \cdots\left(n_{l}+v\right)^{\beta_{l}(z)}$ is holomorphic at $z=0$.

Now set $m=0$ in the equation above. The terms (70) and (71) still vanish, and only $p=0$ and $p=1$ give nonzero contribution in the terms (72), (73) and (74) according to the induction hypothesis. For $p=1$, these three terms are rational expressions in $c_{1}, \ldots, c_{l}$ and in the first derivative of $\gamma$ at $z=0$ (which is 1 by hypothesis). For $p=0$ this is still the case for (72) and (73) as well as for the terms in the sum (744) corresponding to $j \leq b_{l}+b_{l-1}+1$, again due to the induction hypothesis. The other terms in the sum (74) do not contribute as $H_{l}^{j}(0)=0$ for $j \geq b_{l}+1$.

- Now suppose $b_{l} \leq-1$ and suppose $m \geq 2$ in the equation above. The terms (72), (73) and (744) vanish according to the induction hypothesis. The terms (70) and (711) also vanish unless possibly when $b_{l}=-1$. But in that case we have $b_{l-1}+b_{l}+1=b_{l-1} \geq 0$, and these two terms also vanish in view of the induction hypothesis. Hence the pole is of order at most one.

Finally set $m=1$ in the equation above. Only $p=0$ gives a nonzero contribution in the terms (72), (73) and (74) according to the induction hypothesis. The residue $\operatorname{Res}_{z=0}^{1}$ is then a rational expression in $c_{1}, \ldots, c_{l}$ independent of $\gamma$ according to the induction hypothesis and the explicit expression of the residues of $H_{l}^{0}$ and $K_{l}$ at $z=0$.

Theorem 10 Let $v \in \mathbb{R}^{+}$and let $\mathcal{R}_{0, v}: \tau \mapsto\left(\xi \mapsto \tau(z)(\xi)=\tau(\xi)(\xi+v)^{-z}\right)$ be the ordinary Riesz regularisation shifted by $v$, and let $\mathcal{R}_{v}(\tau)(z):=\mathcal{R}_{0, v}(\tau)(\gamma(z))$ where $\gamma$ is a holomorphic function of $z$ with $\gamma^{\prime}(0)=1$. Let us set $\sigma_{s_{i}}^{v}(\xi)=\chi(\xi)(\xi+v)^{-s_{i}}$ and $\sigma^{v}:=\sigma_{-a_{1}}^{v} \otimes \cdots \otimes \sigma_{-a_{k}}^{v}$. At non-positive integer arguments $s_{1}=-a_{1}, \ldots, s_{k}=-a_{k}$, the map

$$
z \mapsto \sum_{<}^{\text {Chen }} \tilde{\mathcal{R}}_{v}^{*}\left(\sigma^{v}\right)(z)
$$

is holomorphic at $z=0$ and the renormalised multiple zeta values

$$
\zeta\left(-a_{1}, \ldots,-a_{k} ; v\right)=\lim _{z \rightarrow 0} \sum_{<}^{\text {Chen }} \tilde{\mathcal{R}}_{v}^{*}\left(\sigma^{v}\right)(z)
$$

is a polynomial of degree $k$ in $v$ with rational coefficients independent of the regularisation function $\gamma$.

Proof: We have $\zeta^{\mathcal{R}_{v}}\left(-a_{1}, \ldots,-a_{k}\right)=\Psi_{+}^{\mathcal{R}_{v}}\left(\sigma^{v}\right)(z)_{z=0}$, where $\Psi^{\mathcal{R}_{v}}(z)\left(\sigma^{v}\right)=\sum_{<}^{\text {Chen }} \widetilde{\mathcal{R}}_{v}^{\star}\left(\sigma^{v}\right)(z)$ is a finite linear combination with rational coefficients of terms of the type:

$$
\sum_{<}^{\text {Chen }} \sigma_{-b_{1}+c_{1} \gamma(z), v} \otimes \cdots \otimes \sigma_{-b_{l}+c_{l} \gamma(z), v}
$$


where $l \in\{1, \ldots, k\}$, where $b_{1}, \ldots, b_{l} \in \mathbb{N}$ and where the $c_{j}$ 's are positive integers. This comes from the explicit expression:

$$
\mathcal{R}_{v}^{\star}\left(\sigma^{v}\right)(z)=\sum_{I=\left(i_{1}, \ldots, i_{r}\right) \in \mathcal{P}(k)} \frac{(-1)^{k-r}}{i_{1} \cdots i_{r}} \sum_{J=\left(c_{1}, \ldots, c_{l}\right) \in \mathcal{P}(r)} \frac{1}{c_{1} ! \cdots c_{l} !} \sigma_{-b_{1}+c_{1} \gamma(z)}^{v} \otimes \cdots \otimes \sigma_{-b_{l}+c_{l} \gamma(z)}^{v},
$$

where $b_{j}$ stands for the sum of the $a_{s}^{\prime} s$ for $s$ inside the $j^{\text {th }}$ packet of the product composition $J \circ I$. The result then follows from Theorem 9 according to which these maps are holomorphic at $z=0$ and their values at $z=0$ rational numbers independent of the choice of $\gamma$. Thus, the restriction of $\Psi^{\mathcal{R}}$ to the Hopf subalgebra $\mathcal{T}\left(\mathcal{A}_{v, \mathbb{N}}\right)$ of $\mathcal{T}\left(\mathcal{A}_{v}\right)$, spanned by $\left\{\sigma_{-a}^{v}: \xi \mapsto \chi(\xi)(\xi+v)^{a}, a \in \mathbb{N}\right\}$, takes its values in holomorphic functions at $z=0$. This implies that $\left.\Psi_{+}^{\mathcal{R}_{v}}\right|_{\mathcal{T}\left(\mathcal{A}_{v, \mathbb{N}}\right)}=\left.\Psi^{\mathcal{R}_{v}}\right|_{\mathcal{T}\left(\mathcal{A}_{v, \mathbb{N}}\right)}$.

Following these steps and using (55), one obtains the following explicit formula for multiple zeta values (i.e. $v=0$ ) at two nonpositive arguments:

$$
\begin{aligned}
\zeta(-a,-b)=\mathrm{fp}_{z=0} \sum_{1 \leq n_{2}<n_{1}} n_{1}^{a-z} n_{2}^{b-z} \\
=\frac{1}{b+1} \sum_{s=0}^{b+1}\left(\begin{array}{c}
b+1 \\
s
\end{array}\right) B_{s} \zeta(-a-b+s-1)+\zeta(-a) \zeta(-b)+(-1)^{a+1} \frac{a ! b !}{2(a+b+2) !} B_{a+b+2} .
\end{aligned}
$$

Formula (77) yields the following table of values $\zeta(-a,-b)$ for $a, b \in\{0, \ldots, 6\}$ :

\begin{tabular}{c|c|c|c|c|c|c|c|}
$\zeta(-a,-b)$ & $a=0$ & $a=1$ & $a=2$ & $a=3$ & $a=4$ & $a=5$ & $a=6$ \\
\hline$b=0$ & $\frac{3}{8}$ & $\frac{1}{12}$ & $\frac{7}{720}$ & $-\frac{1}{120}$ & $-\frac{11}{2520}$ & $\frac{1}{252}$ & $\frac{1}{224}$ \\
\hline$b=1$ & $\frac{1}{24}$ & $\frac{1}{288}$ & $-\frac{1}{240}$ & $-\frac{19}{10080}$ & $\frac{1}{504}$ & $\frac{41}{20160}$ & $-\frac{1}{480}$ \\
\hline$b=2$ & $-\frac{7}{720}$ & $-\frac{1}{240}$ & 0 & $\frac{1}{504}$ & $\frac{113}{151200}$ & $-\frac{1}{480}$ & $-\frac{307}{166320}$ \\
\hline$b=3$ & $-\frac{1}{240}$ & $\frac{1}{840}$ & $\frac{1}{504}$ & $\frac{1}{28800}$ & $-\frac{1}{480}$ & $-\frac{281}{332640}$ & $\frac{1}{264}$ \\
\hline$b=4$ & $\frac{11}{2520}$ & $\frac{1}{504}$ & $-\frac{113}{151200}$ & $-\frac{1}{480}$ & 0 & $\frac{1}{264}$ & $\frac{117977}{75675600}$ \\
\hline$b=5$ & $\frac{1}{504}$ & $-\frac{103}{60480}$ & $-\frac{1}{480}$ & $\frac{1}{1232}$ & $\frac{1}{264}$ & $\frac{1}{127008}$ & $-\frac{691}{65520}$ \\
\hline$b=6$ & $-\frac{1}{224}$ & $-\frac{1}{480}$ & $\frac{307}{166320}$ & $\frac{1}{264}$ & $-\frac{117977}{75675600}$ & $-\frac{691}{65520}$ & 0 \\
\hline
\end{tabular}


Remark 8 If $a+b$ is odd and $b \neq 0$, all terms in equation (77) vanish except the one with $s=1$. This yields:

$$
\zeta(-a,-b)=-\frac{1}{2} \zeta(-a-b),
$$

a fact also established by L. Guo and B. Zhang by different means [GZ]. We also have for odd $a$ :

$$
\zeta(-a, 0)=-\zeta(-a)
$$

This is due to the fact that both versions of the renormalised value coincide with the analytic continuation of the double zeta function, which is holomorphic at those points. As a consequence of the quasi-shuffle relations, the table of values in [GZ] also matches with ours on the diagonal points $(-a,-a)$. It however diverges from ours at other singular points.

\subsection{An alternative renormalization method}

An alternative proposal for multiple zeta values at non-positive arguments can be found in AET (Remark 2 therein) and easily extended to Hurwitz multiple zeta values at non-positive arguments defining as follow:?

$$
\zeta^{\text {alt }}\left(-a_{1}, \ldots,-a_{k} ; v\right):=\lim _{z \rightarrow 0} \zeta\left(-a_{1}+z, \ldots,-a_{k}+z ; v\right)
$$

This uses the $\widetilde{\mathcal{R}}$ regularisation instead of our twisted $\widetilde{\mathcal{R}}^{\star}$ regularisation, so that the stuffle relations are not expected to hold. Multiple Hurwitz zeta functions at nonpositive integer arguments $-a_{1}, \ldots,-a_{k}$ are a priori sensitive to the twisting of the regularisation by the Hoffman isomorphism because of the dependence on the coefficients $c_{1}>0, \ldots, c_{k}>0$ of the $\operatorname{limit}_{\lim _{z \rightarrow 0}} \zeta\left(-a_{1}+c_{1} z, \ldots,-a_{k}+c_{k} z ; v\right)$ studied in Theorem 9. They nevertheless coincide for depths 1 and 2:

Proposition 13 For any non positive integers $a_{1}$ and $a_{2}$ and any non negative real number $v$, we have:

$$
\zeta\left(-a_{1} ; v\right)=\zeta^{\text {alt }}\left(-a_{1} ; v\right) \text { and } \quad \zeta\left(-a_{1},-a_{2} ; v\right)=\zeta^{\text {alt }}\left(-a_{1},-a_{2}: v\right) .
$$

Proof: This follows from the holomorphicity of the ordinary Hurwitz zeta function at non-positive integers.

However, the two methods are expected to differ for depth $k>2$; this is confirmed in the depth 3 case investigated in the following example:

Example 2 For $v=0$ and any three symbols $\sigma_{1}, \sigma_{2}, \sigma_{3}$ corresponding to non-positive integer arguments $s_{1}=-a_{1}, s_{2}=-a_{2}, s_{3}=-a_{3}$, one infers from the explicit formulae for Hoffman's log and exp given in Section 3.1 that:

$$
\begin{aligned}
& \tilde{\mathcal{R}}^{*}\left(\sigma_{1} \otimes \sigma_{2} \otimes \sigma_{3}\right)(z)-\widetilde{\mathcal{R}}\left(\sigma_{1} \otimes \sigma_{2} \otimes \sigma_{3}\right)(z) \\
= & \frac{1}{2}\left(\sigma_{1}(z) \sigma_{2}(z) \otimes \sigma_{3}(z)+\sigma_{1}(z) \otimes \sigma_{2}(z) \sigma_{3}(z)-\left(\sigma_{1} \sigma_{2}\right)(z) \otimes \sigma_{3}(z)-\sigma_{1}(z) \otimes\left(\sigma_{2} \sigma_{3}\right)(z)\right) \\
+ & \frac{1}{6} \sigma_{1}(z) \sigma_{2}(z) \sigma_{3}(z)-\frac{1}{4}\left(\sigma_{1} \sigma_{2}\right)(z) \sigma_{3}(z)-\frac{1}{4} \sigma_{1}(z)\left(\sigma_{2} \sigma_{3}\right)(z)+\frac{1}{3}\left(\sigma_{1} \sigma_{2} \sigma_{3}\right)(z),
\end{aligned}
$$

${ }^{9}$ modulo the reverse convention in the order of the arguments 
so that, using the holomorphicity of the zeta function at non-positive integer arguments, we get

$$
\begin{gathered}
\Delta\left(-a_{1},-a_{2},-a_{3}\right)=\zeta\left(-a_{1},-a_{2},-a_{3}\right)-\zeta^{\text {alt }}\left(-a_{1},-a_{2},-a_{3}\right) \\
=\lim _{z \rightarrow 0} \frac{1}{2}\left(\zeta\left(-a_{1}-a_{2}+2 z,-a_{3}+z\right)+\zeta\left(-a_{1}+z,-a_{2}-a_{3}+2 z\right)-\zeta\left(-a_{1}-a_{2}+z,-a_{3}+z\right)-\zeta\left(-a_{1}+z,-a_{2}-a_{3}+z\right)\right) \\
\quad+\lim _{z \rightarrow 0}\left[\frac{1}{6} \zeta\left(-a_{1}-a_{2}-a_{3}+3 z\right)-\frac{1}{4} \zeta\left(-a_{1}-a_{2}-a-a_{3}+2 z\right)-\frac{1}{4} \zeta\left(-a_{1}-a_{2}-a_{3}+2 z\right)+\frac{1}{3} \zeta\left(-a_{1}-a_{2}-a_{3}+z\right)\right] \\
=\lim _{z \rightarrow 0} \frac{1}{2}\left(\zeta\left(-a_{1}-a_{2}+2 z,-a_{3}+z\right)+\zeta\left(-a_{1}+z,-a_{2}-a_{3}+2 z\right)-\zeta\left(-a_{1}-a_{2}+z,-a_{3}+z\right)-\zeta\left(-a_{1}+z,-a_{2}-a_{3}+z\right)\right) .
\end{gathered}
$$

In particular, we have

$$
\begin{aligned}
& \Delta(0,-1,-1 ; v)=\zeta(0,-1,-1 ; v)-\zeta^{\text {alt }}(0,-1,-1 ; v) \\
= & \frac{1}{2} \operatorname{fp}_{z \rightarrow 0} \operatorname{fp}_{N \rightarrow \infty}\left(\sum_{n_{1}=1}^{N} \sum_{n_{2}=1}^{n_{1}-1}\left(n_{1}+v\right)^{1-2 z}\left(n_{2}+v\right)^{1-z}+\sum_{n_{1}=1}^{N} \sum_{n_{2}=1}^{n_{1}-1}\left(n_{1}+v\right)^{-z}\left(n_{2}+v\right)^{2-2 z}\right) \\
& -\frac{1}{2} \operatorname{fp}_{z \rightarrow 0} \operatorname{fp}_{N \rightarrow \infty}\left(\sum_{n_{1}=1}^{N} \sum_{n_{2}=1}^{n_{1}-1}\left(n_{1}+v\right)^{1-z}\left(n_{2}+v\right)^{1-z}-\sum_{n_{1}=1}^{N} \sum_{n_{2}=1}^{n_{1}-1}\left(n_{1}+v\right)^{-z}\left(n_{2}+v\right)^{2-z}\right) \\
= & \frac{B_{4}}{288}(2-8-3+6) \neq 0 .
\end{aligned}
$$

Remarkably enough, the result does not depend on $v$.

\subsection{Two identities preserved at nonpositive arguments}

Hurwitz multiple zeta functions verify the following two identities on the domain of convergence, namely for any $\left(s_{1}, \ldots, s_{k}\right) \in \mathbb{C}^{k}$ with $\operatorname{Re}\left(s_{1}+\cdots+s_{m}\right)>m$ for all $m \in\{1, \ldots, k\}:$

$$
\zeta\left(s_{1}, \ldots, s_{k} ; v+1\right)=\zeta\left(s_{1}, \ldots, s_{k} ; v\right)-(v+1)^{-s_{k}} \zeta\left(s_{1}, \ldots, s_{k-1} ; v+1\right)
$$

and

$$
\frac{d}{d v} \zeta\left(s_{1}, \ldots, s_{k} ; v\right)=\sum_{j=1}^{k}-s_{j} \zeta\left(s_{1}, \ldots, s_{j-1}, s_{j}+1, s_{j+1}, \ldots, s_{k} ; v\right) .
$$

The proof is straightforward and left to the reader. We show here that both identities are preserved at nonpositive integer arguments, for both renormalisations $\zeta$ and $\zeta^{\text {alt }}$.

Proposition 14 For any $a_{1}, \ldots, a_{k} \in \mathbb{N}$ we have:

$\zeta^{\text {alt }}\left(-a_{1}, \ldots,-a_{k} ; v+1\right)=\zeta^{\text {alt }}\left(-a_{1}, \ldots,-a_{k} ; v\right)-(v+1)^{a_{k}} \zeta^{\text {alt }}\left(-a_{1}, \ldots,-a_{k-1} ; v+1\right)$

and moreover if $a_{1}, \ldots, a_{k} \geq 1$ :

$$
\frac{d}{d v} \zeta^{\text {alt }}\left(-a_{1}, \ldots,-a_{k} ; v\right)=-\sum_{j=1}^{k} a_{j} \zeta^{\text {alt }}\left(-a_{1}, \ldots,-a_{j-1},-a_{j}+1,-a_{j+1}, \ldots,-a_{k} ; v\right) .
$$


Proof: This follows from the two identities of meromorphic functions (with respect to the variables $s_{j}$ ):

$$
\zeta\left(s_{1}, \ldots, s_{k} ; v+1\right)=\zeta\left(s_{1}, \ldots, s_{k} ; v\right)-(v+1)^{-s_{k}} \zeta\left(s_{1}, \ldots, s_{k-1} ; v+1\right)
$$

and:

$$
\frac{d}{d v} \zeta\left(s_{1}, \ldots, s_{k} ; v\right)=-\sum_{j=1}^{k} s_{j} \zeta\left(s_{1}, \ldots, s_{j-1}, s_{j}+1, s_{j+1}, \ldots, s_{k} ; v\right) .
$$

which in turn yield the following identities of holomorphic functions:

$\zeta\left(-a_{1}+z, \ldots,-a_{k}+z ; v+1\right)=\zeta\left(-a_{1}+z, \ldots,-a_{k}+z ; v\right)-(v+1)^{a_{k}+z} \zeta\left(-a_{1}+z, \ldots,-a_{k-1}+z ; v+1\right)$

and:

$$
\frac{d}{d v} \zeta\left(-a_{1}+z, \ldots,-a_{k}+z ; v\right)=\sum_{j=1}^{k} a_{j} \zeta\left(-a_{1}, \ldots,-a_{j-1},-a_{j}+1,-a_{j+1}, \ldots,-a_{k} ; v\right) .
$$

Theorem 11 For any $a_{1}, \ldots, a_{k} \in \mathbb{N}$ we have:

$$
\zeta\left(-a_{1}, \ldots,-a_{k} ; v+1\right)=\zeta\left(-a_{1}, \ldots,-a_{k} ; v\right)-(v+1)^{a_{k}} \zeta\left(-a_{1}, \ldots,-a_{k-1} ; v+1\right)
$$

and moreover when $a_{1}, \ldots, a_{k} \geq 1$ :

$$
\frac{d}{d v} \zeta\left(-a_{1}, \ldots,-a_{k} ; v\right)=-\sum_{j=1}^{k} a_{j} \zeta\left(-a_{1}, \ldots,-a_{j-1},-a_{j}+1,-a_{j+1}, \ldots,-a_{k} ; v\right) .
$$

Proof: Equation (88) is equivalent to:

$$
\zeta\left(-a_{1}, \ldots,-a_{k} ; v+1\right)=\sum_{r=0}^{k}(-1)^{r}(v+1)^{a_{k}+\cdots+a_{k-r+1}} \zeta\left(-a_{1}, \ldots,-a_{k-r} ; v\right),
$$

where the term corresponding to $r=0$ (resp. $r=k$ ) is $\zeta\left(-a_{1}, \ldots,-a_{k} ; v\right.$ ) (resp. $\left.(-1)^{k}(v+1)^{a_{k}+\cdots+a_{1}}\right)$. We prove (90) by induction on the depth $k$. We denote by $\delta_{1}$ any positively supported symbol such that $\delta_{1}(1)=1$ and $\delta_{1}(\xi)=0$ for $\xi \geq 2$. We set $\sigma^{v}(\xi):=\sigma(\xi+v)$ for any symbol $\sigma$, as well as $(\sigma \otimes \tau)^{v}:=\sigma^{v} \otimes \tau^{v}$ and so on. Using 
the notations of Theorem 10 we compute:

$$
\begin{aligned}
& \zeta\left(-a_{1}, \ldots,-a_{k} ; v\right)-\zeta\left(-a_{1}, \ldots,-a_{k} ; v+1\right) \\
= & \lim _{z \rightarrow 0} \sum^{\text {Chen }} \widetilde{\mathcal{R}}_{v}^{*}\left(\sigma_{s_{1}}^{v} \otimes \cdots \otimes \sigma_{s_{k}}^{v}\right)(z)-\widetilde{\mathcal{R}}_{v+1}^{*}\left(\sigma_{s_{1}}^{v+1} \otimes \cdots \otimes \sigma_{s_{k}}^{v+1}\right)(z) \\
= & \lim _{z \rightarrow 0} \sum^{\text {Chen }}\left(\widetilde{\mathcal{R}}_{0}^{*}\left(\sigma_{s_{1}} \otimes \cdots \otimes \sigma_{s_{k}}\right)(z)^{v}-\widetilde{\mathcal{R}}_{0}^{*}\left(\sigma_{s_{1}} \otimes \cdots \otimes \sigma_{s_{k}}\right)(z)^{v+1}\right) \\
= & \lim _{z \rightarrow 0} \sum^{\text {Chen }} \widetilde{\mathcal{R}}_{v}^{*}\left(\sigma_{s_{1}}^{v} \otimes \cdots \otimes \sigma_{s_{k-1}}^{v} \otimes \sigma_{s_{k}}^{v} \delta_{1}\right)(z) \\
= & \lim _{z \rightarrow 0} \sum^{\text {Chen }} \widetilde{\mathcal{R}}_{v}^{*}\left(\left(\sigma_{s_{1}}^{v} \otimes \cdots \otimes \sigma_{s_{k-1}}^{v}\right) \star \sigma_{s_{k}}^{v} \delta_{1}\right)(z)-\lim _{z \rightarrow 0} \sum^{\text {Chen }} \widetilde{\mathcal{R}}_{v}^{*}\left(\left(\sigma_{s_{1}}^{v} \otimes \cdots \otimes \sigma_{s_{k-2}}^{v}\right) \otimes \sigma_{s_{k-1}}^{v} \sigma_{s_{k}}^{v} \delta_{1}\right)(z) \\
= & \sum_{r=1}^{k}(-1)^{r-1} \lim _{z \rightarrow 0} \sum^{\mathrm{Chen}} \widetilde{\mathcal{R}}_{v}^{*}\left(\left(\sigma_{s_{1}}^{v} \otimes \cdots \otimes \sigma_{s_{k-r}}^{v}\right) \star\left(\sigma_{s_{k-r+1}}^{v} \cdots \sigma_{s_{k}}^{v} \delta_{1}\right)\right)(z) \\
= & \sum_{r=1}^{k}(-1)^{r-1}(v+1)^{a_{k}+\cdots+a_{k-r+1}} \lim _{z \rightarrow 0} \sum^{\mathrm{Chen}} \widetilde{\mathcal{R}}_{v}^{*}\left(\sigma_{s_{1}}^{v} \otimes \cdots \otimes \sigma_{s_{k-r}}^{v}\right)(z) \\
= & \sum_{r=1}^{k}(-1)^{r-1}(v+1)^{a_{k}+\cdots+a_{k-r+1}} \zeta\left(-a_{1}, \ldots,-a_{k-r} ; v\right) .
\end{aligned}
$$

Here we have used Theorem [10 and the expression (76) implicitly. Equation (89) is proved as follows, still applying Theorem [10. Here $a_{1}, \ldots, a_{k}$ are positive integers (i.e. $\geq 1$ ) with $s_{j}=-a_{j}, j=1, \ldots, k$, and the operator $\frac{d}{d v}$ will also be denoted by a dot:

$$
\begin{aligned}
& \frac{d}{d v} \zeta\left(-a_{1}, \ldots,-a_{k} ; v\right)=\frac{d}{d v}\left(\left.\sum^{\text {Chen }} \widetilde{\mathcal{R}}_{v}^{*}\left(\sigma_{s_{1}}^{v} \otimes \cdots \otimes \sigma_{s_{k}}^{v}\right)(z)\right|_{z=0}\right) \\
= & \left.\left(\frac{d}{d v} \sum^{\text {Chen }} \widetilde{\mathcal{R}}_{v}^{*}\left(\sigma_{s_{1}}^{v} \otimes \cdots \otimes \sigma_{s_{k}}^{v}\right)(z)\right)\right|_{z=0}(\text { by holomorphy at } z=0) \\
= & \left.\left(\sum^{\text {Chen }} \frac{d}{d v} \widetilde{\mathcal{R}}_{v}^{*}\left(\sigma_{s_{1}}^{v} \otimes \cdots \otimes \sigma_{s_{k}}^{v}\right)(z)\right)\right|_{z=0} \\
= & \left.\left(\sum^{\text {Chen }} \frac{d}{d v} \exp \circ \widetilde{\mathcal{R}}_{v} \circ \log \left(\sigma_{s_{1}}^{v} \otimes \cdots \otimes \sigma_{s_{k}}^{v}\right)(z)\right)\right|_{z=0} \\
= & \left.\left(\sum^{\text {Chen }}\left(\exp \circ \dot{\widetilde{\mathcal{R}}}_{v} \circ \log \left(\sigma_{s_{1}}^{v} \otimes \cdots \otimes \sigma_{s_{k}}^{v}\right)(z)+\sum_{j=0}^{k} \exp \circ \widetilde{\mathcal{R}}_{v} \circ \log \left(\sigma_{s_{1}}^{v} \otimes \cdots \otimes \dot{\sigma}_{s_{j}}^{v} \otimes \cdots \otimes \sigma_{s_{k}}^{v}\right)(z)\right)\right)\right|_{z=0} \\
= & -\left.\sum_{j=1}^{k}\left(z+s_{j}\right)\left(\sum^{\text {Chen }} \exp \circ \widetilde{\mathcal{R}}_{v} \circ \log \left(\sigma_{s_{1}}^{v} \otimes \cdots \otimes \sigma_{s_{j}+1}^{v} \otimes \cdots \otimes \sigma_{s_{k}}^{v}\right)(z)\right)\right|_{z=0} \\
= & \sum_{j=1}^{k} a_{j} \zeta\left(-a_{1}, \ldots,-a_{j-1},-a_{j}+1,-a_{j+1}, \ldots, a_{k} ; v\right) .
\end{aligned}
$$




\section{A HIGHER DIMENSIONAL ANALOG OF MUL- TIPLE ZETA FUNCTIONS}

Using radial symbols we extend the constructions carried out previously in the onedimensional case to a higher dimensional setup using nested cubes. Proposition ?? and Theorem 12 relate the higher dimensional multiple zeta functions to the onedimensional ones, thus enabling us to carry out to this higher dimensional setting the techniques implemented in the one dimensional case.

\subsection{An algebra of radial symbols}

Let $|\cdot|$ be any norm on $\mathbb{R}^{n}$. Let $\bullet$ be the product on complex valued functions on $\mathbb{Z}^{n}$ vanishing at the origin, defined by

$$
\sigma_{1} \bullet \sigma_{2}(x):=\frac{1}{A_{n}(|x|)} \sum_{\left|x_{1}\right|=\left|x_{2}\right|=|x|} \sigma_{1}\left(x_{1}\right) \cdot \sigma_{2}\left(x_{2}\right) \quad \forall x \in \mathbb{Z}^{n},
$$

where for a non-negative number $t=|x|$ for some $x \in \mathbb{Z}^{n}$, the number of points with integer coordinates in the sphere centered at zero of radius $t$ is denoted by $A_{n}(t)$. We have $A_{n}(0)=1$ and, when $|\cdot|$ is the supremum norm $|x|=\sup _{i=1}^{n}\left|x_{i}\right|$ on $\mathbb{R}^{n}$, then $A_{n}(t)=(2 t+1)^{n}-(2 t-1)^{n}$ for any positive integer $t$. The product $\bullet$ is commutative and associative as a result of the commutativity and associativity of the ordinary product.

Definition 12 For any complex valued function $\sigma$ on $\mathbb{Z}^{n}$ and any $x \in \mathbb{Z}^{n}$ let us set:

$$
R_{n}(\sigma)(x):=\sum_{0<|y|<|x|, y \in \mathbb{Z}^{n}} \sigma(y)
$$

This construction can be immediately extended to complex valued functions on $\mathbb{R}^{n}$ provided the function $A_{n}$ is suitably interpolated to all positive real numbers by a nowhere vanishing function. We now specialise to radial functions $\sigma=f \circ|\cdot|$ on $\mathbb{R}^{n}$ in which case the product $\bullet$ reads:

$$
\sigma_{1} \bullet \sigma_{2}(x)=A_{n}(|x|) \sigma_{1}(x) \sigma_{2}(x) .
$$

The radial part $\rho_{n}$ of the operator $R_{n}$, defined by:

$$
R_{n}(f \circ|\cdot|)=\rho_{n}(f) \circ|\cdot|
$$

reads as follows:

$$
\rho_{n}(f)(N):=\sum_{0<p<N} A_{n}(p) f(p)
$$

where the sum runs over all positive real numbers $p$ such that the sphere of radius $p$ has non-empty intersection with $\mathbb{Z}^{n}$. Let $\mathcal{P}_{1}=\mathcal{P}^{*, 0}$ be the algebra of positively supported classical symbols, let $\mathcal{A}$ be a subalgebra of $\mathcal{P}_{1}$, and let $M$ the linear operator on $\mathcal{A}$ with values into radial functions given by:

$$
M(f):=\frac{f}{A_{n}} \circ|\cdot| .
$$


Let $\mathcal{A}_{n}$ be the image of $\mathcal{A}$ by the operator $M$; by (93) we have $M\left(f_{1} f_{2}\right)=M\left(f_{1}\right) \bullet$ $M\left(f_{2}\right)$ so that $M$ yields an algebra isomorphism from $(\mathcal{A},$.$) onto \left(\mathcal{A}_{n}, \bullet\right)$. This induces a Hopf algebra isomorphism

$$
\mathcal{M}:\left(\mathcal{H}_{\infty}, \star\right) \rightarrow\left(\mathcal{H}_{\curlyvee}, \star_{\bullet}\right)
$$

between the two corresponding quasi-shuffle Hopf algebras.

\subsection{Higher-dimensional renormalised multiple zeta values}

We now specialise to the supremum norm $|x|=\sup _{i=1}^{n}\left|x_{i}\right|$ on $\mathbb{R}^{n}$. The reason for this is that the image of $\mathbb{Z}^{n}$ by this norm is equal to $\mathbb{N}$, so that sums over $\mathbb{Z}^{n}$ boil down to sums over the integers. The methods developped previously in the one-dimensional situation can then be transposed to the $n$-dimensional one by means of the Hopf algebra isomorphism $\mathcal{M}$ defined in the previous paragraph. We do not know at this stage how to build meromorphic extensions of multiple zeta functions for radial symbols when $|\cdot|$ is the Euclidean norm since we do not know how to count the integer points inside the unit sphere.

We interpolate the number of points with integer coordinates on spheres by setting:

$$
A_{n}(t)=(2 t+1)^{n}-(2 t-1)^{n}
$$

for any $t \geq 0$. The vanishing of this interpolated $A_{n}$ at $t=0$ is harmless as we deal with symbols which vanish at the origin.

Definition 13 Let $\mathcal{A}$ be a subalgebra of $\mathcal{P}_{1}$ and let $\mathcal{R}$ be a holomorphic regularisation on $\mathcal{A}$. Let $\sum_{<}^{\text {Chen, } \mathcal{R}}$ be the renormalised nested sum character of Definition 10 (in the strict inequality version) on $\mathcal{H}_{1}=\mathcal{T}(\mathcal{A})$. The higher-dimensional renormalised nested sum character on radial symbols is defined by:

$$
\sum_{<}^{\text {Chen, } \mathcal{R}, n} \sigma:=\sum_{<}^{\text {Chen, } \mathcal{R}} \mathcal{M}^{-1}(\sigma) .
$$

for any $\sigma \in \mathcal{H}_{n}$.

The fact that this defines a character for the $\star_{\bullet}$ quasi-shuffle product on $\mathcal{H}_{n}$ follows from

$$
\sum_{<}^{\text {Chen, } \mathcal{R}} \mathcal{M}^{-1}(\sigma)=\psi^{\mathcal{R}} \circ \mathcal{M}^{-1}(\sigma)
$$

where $\psi^{\mathcal{R}}:\left(\mathcal{H}_{1}, \star\right) \rightarrow \mathbb{C}$ is the Birkhoff-Hopf character introduced in Theorem 7 . A similar definition holds for the weak inequality version $\sum_{\leq}^{\operatorname{Chen,} \mathcal{R}, n}:$ details are left to the reader. We now apply the above constructions to symbols $\sigma_{s}^{v} \sim(|\cdot|+v)^{-s}, s \in \mathbb{C}$, $v \in \mathbb{R}_{+}$i.e. to the algebra of radial classical symbols:

$$
\widetilde{\mathcal{A}}_{n}:=\{f \circ|\cdot|, \quad f \in \mathcal{A}\}
$$

where $\mathcal{A}$ was defined in Paragraph 4.1. We set the following definition: 
Definition 14 Let $\mathcal{R}$ be a holomorphic regularisation on $\mathcal{A}$. Given any $s_{i} \in \mathbb{C}, v_{i} \in$ $\mathbb{R}_{+}$and $\sigma_{s_{i}}^{v_{i}}=\left(|\cdot|+v_{i}\right)^{-s_{i}}$ for some non negative real number $v$, we define the renormalised multiple Hurwitz zeta values at $\left(s_{1}, \ldots, s_{k}\right)$ by

$$
\zeta_{n}^{\mathcal{R}}\left(s_{1}, \ldots, s_{k} ; v_{1}, \ldots, v_{k}\right):=\sum_{<}^{\text {Chen, } \mathcal{R}, n} \sigma_{s_{1}}^{v_{1}} \otimes \cdots \otimes \sigma_{s_{k}}^{v_{k}}
$$

where the renormalised nested sum is taken as in Definition 13. As in the onedimensional case, we denote by $\zeta_{n}^{\mathcal{R}}\left(s_{1}, \ldots, s_{k} ; v\right)$ the Hurwitz multiple zeta value with parameters $v_{1}=\cdots=v_{k}=v$ and define renormalised higher-dimensional multiple zeta values by setting the parameters $v_{i}$ to zero:

$$
\zeta_{n}^{\mathcal{R}}\left(s_{1}, \ldots, s_{k}\right):=\zeta_{n}^{\mathcal{R}}\left(s_{1}, \ldots, s_{k} ; 0, \ldots, 0\right)
$$

\subsection{Explicit formulae for higher-dimensional multiple zeta val- ues}

The linear map

$$
\begin{aligned}
N: \mathcal{A} & \rightarrow \widetilde{\mathcal{A}}_{n} \\
f & \mapsto N(f):=f \circ|\cdot|
\end{aligned}
$$

where as before $|\cdot|$ stands for the supremum norm, induces an isomorphism of tensor algebras $\mathcal{N}: \mathcal{T}(\mathcal{A}) \rightarrow \mathcal{T}\left(\widetilde{\mathcal{A}}_{n}\right)$. On the other hand, the composition $M \circ N^{-1}:$ $\left(\widetilde{\mathcal{A}}_{n}, \cdot\right) \rightarrow\left(\mathcal{A}_{n}, \bullet\right)$ yields an isomorphism of algebras. Let us observe that

$$
\begin{aligned}
\zeta_{n}^{\mathcal{R}}\left(s_{1}, \ldots, s_{k} ; v_{1}, \ldots, v_{k}\right) & =\sum_{<}^{\text {Chen, } \mathcal{R}} \mathcal{M}^{-1} \circ \mathcal{N}\left(f_{s_{1}}^{v_{1}} \otimes \cdots \otimes f_{s_{k}}^{v_{k}}\right) \\
& =\sum_{<}^{\text {Chen, } \mathcal{R}} A_{n} f_{s_{1}}^{v_{1}} \otimes \cdots \otimes A_{n} f_{s_{k}}^{v_{k}}
\end{aligned}
$$

where we have set $f_{s_{i}}^{v_{i}}(t):=\left(t+v_{i}\right)^{-s_{i}}$ and where $A_{n}$ was defined in Paragraph 5.1. Equation (101) yields the following explicit expression for higher-dimensional renormalised multiple zeta values (here $v=0$ ):

Theorem 12

$$
\zeta_{n}^{\mathcal{R}}\left(s_{1}, \ldots, s_{k}\right)=\sum_{J=\left(j_{1}, \ldots, j_{k}\right), j_{r}=0, \ldots,\left[\frac{n}{2}\right]} 2^{n k-2 \mid J]}\left(\begin{array}{c}
n \\
2 j_{1}+1
\end{array}\right) \cdots\left(\begin{array}{c}
n \\
2 j_{k}+1
\end{array}\right) \zeta^{\mathcal{R}}\left(s_{1}-n+2 j_{1}+1, \ldots, s_{k}-n+2 j_{k}+1\right) .
$$

Examples of particular instances of (102) are:

$$
\begin{aligned}
\zeta_{1}^{\mathcal{R}}(s) & =2 \zeta^{\mathcal{R}}(s) \\
\zeta_{2}^{\mathcal{R}}(s) & =8 \zeta^{\mathcal{R}}(s-1) \\
\zeta_{3}^{\mathcal{R}}(s) & =24 \zeta^{\mathcal{R}}(s-2)+2 \zeta^{\mathcal{R}}(s) \\
\zeta_{1}^{\mathcal{R}}\left(s_{1}, s_{2}\right) & =4 \zeta^{\mathcal{R}}\left(s_{1}, s_{2}\right) \\
\zeta_{2}^{\mathcal{R}}\left(s_{1}, s_{2}\right) & =64 \zeta^{\mathcal{R}}\left(s_{1}-1, s_{2}-1\right) \\
\zeta_{3}^{\mathcal{R}}\left(s_{1}, s_{2}\right) & =576 \zeta^{\mathcal{R}}\left(s_{1}-2, s_{2}-2\right)+48 \zeta^{\mathcal{R}}\left(s_{1}-2, s_{2}\right)+48 \zeta^{\mathcal{R}}\left(s_{1}-2, s_{2}\right)+4 \zeta^{\mathcal{R}}\left(s_{1}, s_{2}\right)
\end{aligned}
$$




\subsection{Higher-dimensional Hurwitz multiple zeta values renor- malized at non positive integers}

Since $A_{n}(t)$ is polynomial in $t$ with integer coefficients:

$$
A_{n}(t)=\sum_{j=0}^{\left[\frac{n}{2}\right]} 2^{n-2 j}\left(\begin{array}{c}
n \\
2 j+1
\end{array}\right) t^{n-2 j-1}
$$

it can be written as a polynomial in the variable $t+v$ with coefficients given by polynomials in $v$ with integer coefficients. Thus, when $v_{1}=\cdots=v_{k}=v$ the tensor product $A_{n} f_{s_{1}}^{v_{1}} \otimes \cdots \otimes A_{n} f_{s_{k}}^{v_{k}}$ can be written as a finite linear combination of tensor products $f_{s_{1}-b_{1}}^{v_{1}} \otimes \cdots \otimes f_{s_{k}-b_{k}}^{v_{k}}$ with coefficients given by polynomials in $v$ with integer coefficients and for some non negative integers $b_{1}, \ldots, b_{k}$. By Theorem [10, setting $v_{i}=v$ and $s_{i}=-a_{i}$ for any $i \in\{1, \cdots, k\}$, for non negative integers $a_{i}$ the expression Chen, $\mathcal{R}$

$\sum_{<}^{\text {Chen } \mathcal{R}} f_{s_{1}-b_{1}}^{v_{1}} \otimes \cdots \otimes f_{s_{k}-b_{k}}^{v_{k}}$ is a polynomial expression in $v$ with rational coefficients, hence following result.

Proposition 15 Multiple zeta values $\zeta_{n}^{\mathcal{R}}\left(-a_{1}, \ldots,-a_{k} ; v\right)$ at non positive integers are polynomials with rational coefficients in $v$. In particular, higher-dimensional multiple zeta values are rational at nonpositive arguments. 


\section{Appendix: A continuous analog of polylogarithms}

We describe a continuous analog of polylogarithms similar to their discrete counterparts in number theory. In contrast to the latter, the shuffle relations obtained here are of the same type as the original ones but they hold on a different Hopf algebra. We define a continuous analog of polylogarithms setting for $1 \geq r>0,|z|<1, s_{1}>1$ and $s_{i} \geq 1$ for $1<i \leq k$ :

$$
\tilde{L} i_{\underline{s}}^{r}(z)=\int_{r \leq t_{k} \leq \cdots \leq t_{1}} d t_{1} \cdots d t_{k} z^{t_{1}} \prod_{i=1}^{k} t_{i}^{-s_{i}} .
$$

Note that

$$
\tilde{L} i_{\underline{s}}^{r}(1)=\tilde{\zeta}^{r}(\underline{s}) \text {. }
$$

Proposition 16 Let $\underline{s} \in \mathbb{R}^{k}$. For any $0<z<1$,

1. if $s_{1}=1$ then

$$
\frac{d}{d z} \tilde{L} i_{\underline{s}}(z)=-\frac{1}{z \log z} \tilde{L} i_{s_{2}, \ldots, s_{k}}^{r}(z) .
$$

2. Otherwise

$$
\frac{d}{d z} \tilde{L} i_{\underline{s}}^{r}(z)=\frac{1}{z} \tilde{L} i_{\left(s_{1}-1, s_{2}, \ldots, s_{k}\right)}^{r}(z) .
$$

Proof:

1. Let us first assume that $s_{1}=1$.

$$
\begin{aligned}
\frac{d}{d z} \tilde{L} i_{\underline{s}}^{r}(z) & =\frac{d}{d z} \int_{r \leq t_{k} \leq \cdots \leq t_{2} \leq t_{1}} d t_{1} \cdots d t_{k} \frac{z^{t_{1}}}{t_{1}} \prod_{i=2}^{k} t_{i}^{-s_{i}} \\
& =\frac{d}{d z} \int_{r \leq t_{k} \leq \cdots \leq t_{3} \leq t_{2}} d t_{2} \cdots d t_{k} f_{t_{2}}^{\infty} \frac{z^{t_{1}}}{t_{1}} d t_{1} \prod_{i=2}^{k} t_{i}^{-s_{i}} \\
& =\int_{r \leq t_{k} \leq \cdots \leq t_{3} \leq t_{2}} d t_{2} \cdots d t_{k}\left[\frac{z^{t_{1}-1}}{\log z}\right]_{t_{2}}^{\infty} \prod_{i=2}^{k} t_{i}^{-s_{i}} \\
& =-\frac{1}{z \log z} \tilde{L}_{\left(s_{2}, \ldots, s_{k}\right)}^{r}(z) .
\end{aligned}
$$

2. Let us now assume that $s_{1} \neq 1$.

$$
\begin{aligned}
\frac{d}{d z} \tilde{L} i_{\underline{s}}^{r}(z) & =\frac{d}{d z} \int_{r \leq t_{k} \leq \cdots \leq t_{1}} d t_{1} \cdots d t_{k} z^{t_{1}} \prod_{i=1}^{k} t_{i}^{-s_{i}} \\
& =\frac{1}{z} \int_{r \leq t_{k} \leq \cdots \leq t_{1}} d t_{1} \cdots d t_{k} \frac{z^{t_{1}}}{t_{1}^{s_{1}-1}} \prod_{i=2}^{k} t_{i}^{-s_{i}} \\
& =\frac{1}{z} \tilde{L}_{\left(s_{1}-1, s_{2}, \ldots, s_{k}\right)}^{r}(z) .
\end{aligned}
$$


Following number theorists (see e.g. [C1], ENR, [W], Z]), we now restrict ourselves to positive integer values of the indices $s_{i}$, in which case $\tilde{\zeta}(\underline{s})$ converges if $s_{1}>1$ since all indices $s_{i} \geq 1$. To keep track of the condition $s_{1}>1$ it is useful to introduce a coding of multi-indices $\underline{s}$ by words over the alphabet $X=\left\{x_{0}, x_{1}\right\}$ by the rule:

$$
\underline{s} \mapsto x_{0}^{s_{1}-1} x_{1} x_{0}^{s_{2}-1} x_{1} \cdots x_{0}^{s_{k}-1} x_{1} .
$$

This means that an $x_{1}$ arises every time we have $s_{i}=1$ for some $i$. In contrast, $s_{i}>1$ gives rise to $x_{0}^{s_{i}-1} x_{1}$. Condition $s_{1}>1$ therefore translates to the requirement that the word starts with $x_{0}$. Since the last letter is $x_{1}$ whether $s_{k}=1$ or not, the words arising from a multiindex $\left(s_{1}, \ldots, s_{k}\right)$ must end with $x_{1}$. Following number theorists, we call admissible all words starting with $x_{0}$ and ending with $x_{1}$ and set for any admissible word $w=x_{\underline{s}}$

$$
\tilde{\zeta}_{1}^{r}\left(x_{\underline{s}}\right)=\tilde{\zeta}_{1}^{r}(\underline{s}) .
$$

Clearly, restricting to admissible words amounts to considering only convergent cases. Let us introduce the $\mathbb{Q}$-algebra of polynomials $\mathbb{Q}\langle X\rangle=\mathbb{Q}\left\langle x_{0}, x_{1}\right\rangle$ in two non-commutative variables $x_{0}$ and $x_{1}$ graded by the degree, with $x_{i}$ of degree $1 . \mathbb{Q}\langle X\rangle$ is identified with the graded $\mathbb{Q}$-vector space $\mathcal{H}$ spanned by the monomials in the variables $x_{0}$ and $x_{1}$. We equip $\mathcal{H}$ with the same shuffle product as in the bulk of the paper, defined inductively by:

$$
1 ш w=w \amalg 1=w ; \quad x_{j} u \amalg x_{k} v=x_{j}\left(u \amalg x_{k} v\right)+x_{k}\left(x_{j} u \amalg v\right) .
$$

For any word $w=x_{\underline{s}}$ ending with $x_{1}$ we set 10 :

$$
\tilde{L} i_{x_{\underline{s}}}^{r}(z):=\tilde{L} i_{\underline{s}}^{r}(z) \text {. }
$$

Then the above result translates to:

$$
\frac{d}{d z} \tilde{L} i_{x_{j} u}^{r}(z)=\omega_{j}(z) \tilde{L} i_{u}^{r}(z)
$$

for any non-empty word $u$ and where

1. $\omega_{j}(z)=-\frac{1}{z \log z}$ if $x_{j}=x_{1}$ which corresponds to $s_{1}=1$,

2. and $\omega_{j}(z)=\frac{1}{z}$ if $x_{j}=x_{0}$ which corresponds to $s_{1}>1$.

Note that

$$
\begin{aligned}
\frac{d}{d z} \tilde{L} i_{x_{1}}^{r}(z) & =\frac{d}{d z} \int_{r}^{\infty} \frac{z^{t}}{t} d t=\int_{r}^{\infty} z^{t-1} d t \\
& =\left[\frac{z^{t-1}}{\log z}\right]_{t=r}^{\infty}=-\frac{z^{r-1}}{\log z} .
\end{aligned}
$$

These results motivate the following alternative description of generalised polylogarithms extended to all words: let us set $\tilde{L} i_{1}^{r}(z)=1$ where the subscript 1 denotes the empty word. On the grounds of equation (105) we can inductively extend the polylogarithms from words ending with $x_{1}$ to all words defining them as primitives that

\footnotetext{
${ }^{10}$ Here, as long as $|z|<1$, the restriction on the first letter needed for the convergence of the multiple zeta functions is not necessary anymore.
} 
vanish at $z=0$ (as does the original expression (105)) for words $w=x_{j} u$ containing the letter $x_{1}$ :

$$
\tilde{L} i_{x_{1}}^{r}(z):=\int_{0}^{z} v^{r} \omega_{1}(v), \quad \text { and } \quad \tilde{L}_{x_{j} u}^{r}(z)=\int_{0}^{z} \omega_{j}(v) \tilde{L} i_{u}^{r}(v)
$$

if $u \neq 1$. For words of the form $x_{0}^{s}$ we set:

$$
\tilde{L} i_{x_{0}^{s}}^{r}(z)=\frac{\log ^{s} z}{s !} .
$$

These extended polylogarithms still satisfy equations (105) and therefore coincide with the original polylogarithms for words ending with $x_{1}$.

Theorem 13 The map $w \mapsto \tilde{L} i_{w}^{r}(z)$ is a homomorphism of the algebra $(\mathcal{H}, \amalg)$ into continuous functions on $] 0,1[$. Equivalently,

$$
\tilde{L} i_{w_{1} \amalg w_{2}}^{r}(z)=\tilde{L} i_{w_{1}}^{r}(z) \tilde{L}_{w_{2}}^{r}(z) .
$$

Proof: The proof goes as for usual generalised polylogarithms by a straightforward derivation using (105) (see e.g. Lemma 2 in [Zu]). One shows by induction on the total degree $\left|w_{1}\right|+\left|w_{2}\right|$ that

$$
\frac{d}{d z}\left(\tilde{L} i_{w_{1}}^{r}(z) \tilde{L}_{w_{2}}^{r}(z)\right)=\frac{d}{d z}\left(\tilde{L}_{w_{1} \amalg w_{2}}^{r}(z)\right) .
$$

Hence

$$
\tilde{L} i_{w_{1} ш w_{2}}^{r}(z)=\tilde{L} i_{w_{1}}^{r}(z) \tilde{L} i_{w_{2}}^{r}(z)+C .
$$

The substitution $z=0$ gives $C=0$ for words containing $x_{1}$ and the substitution $z=1$ gives $C=0$ for words built from $x_{0}$ only.

By (105) we obtain the following nested integral representation for the words $w=$ $x_{i_{1}} \cdots x_{i_{k}}$ ending by $x_{1}$ in the domain $0<z<1$ :

$$
\tilde{L} i_{w}^{r}(z)=\int_{0 \leq z_{k} \leq \cdots \leq z_{1} \leq z} \omega_{i_{1}}\left(z_{1}\right) \cdots \omega_{i_{k-1}}\left(z_{k-1}\right) z_{k}^{r} \omega_{i_{k}}\left(z_{k}\right) d z_{1} \cdots d z_{k} .
$$

If $x_{i_{1}} \neq x_{1}$ then this expression is also defined for $z=1$ and provides an alternative nested integral representation for the continuous analog of the multiple zeta functions:

$$
\tilde{\zeta}^{r}(w)=\int_{0 \leq z_{k} \leq \cdots \leq z_{1} \leq 1} \omega_{i_{1}}\left(z_{1}\right) \cdots \omega_{i_{k-1}}\left(z_{k-1}\right) z_{k}^{r} \omega_{i_{k}}\left(z_{k}\right) d z_{1} \cdots d z_{k},
$$

which therefore obey the following shuffle relations:

$$
\tilde{\zeta}^{r}\left(w_{1} \amalg w_{2}\right)=\tilde{\zeta}^{r}\left(w_{1}\right) \tilde{\zeta}^{r}\left(w_{2}\right) .
$$




\section{References}

[AET] S. Akiyama, S. Egami, Y. Tanigawa, Analytic continuation of multiple zeta functions and their values at non-positive integers, Acta Arith. 98, 107-116 (2001).

[C1] P. Cartier, Fonctions polylogarithmes, nombres polyzeta et groupes prounipotents, Séminaire Bourbaki, Astérisque n. 282 (2002) 137-173

[C2] P. Cartier, An introduction to zeta functions, in "From number theory to physics", ed. M. Walschmidt, P. Moussa, J.-M. Luck, C. Itzykson Springer Verlag (1992).

[Ch] K-T. Chen, Iterated path integrals, Bull. Amer. Math. Soc. 83, no. 5, 831-879 (1977).

[CM] A. Connes, M. Marcolli, From Physcs to Number theory via Noncommutative Geometry Frontiers in number theory, physics, and geometry. I, 269-347, Springer, Berlin, 2006

[CEMP] C. Costermans, H.N. Minh, J.Y. Enjalbert, M. Petitot, Structure and asymptotic expansion of multiple harmonic sums, ISSAC Proceedings, Beijing, 24-27 July 2005.

[CK] A. Connes, D. Kreimer, Hopf algebras, Renormalisation and Noncommutative Geometry, Comm. Math. Phys. 199 (1988) 203-242 .

[EG] K. Ebrahimi-Fard, L. Guo, Mixable Shuffles, Quasi-shuffles and Hopf Algebras J. Algebraic Combin. 24, no. 1 (2006) 83-101

[E] J. Ecalle, ARI/GARI, la dimorphie et l'arithmétique des multizêtas : un premier bilan, J. Théor. Nomb. Bordeaux, 15 No 2, 411-478 (2003).

[ENR] M. Espie, J-Ch. Novelli, G. Racinet, Formal computations about multiple zeta values, IRMA Lect. Math. Theor. Phys. 3, Berlin (2003).

[G] A. Goncharov, Multiple polylogarithms and mixed Tate motives, preprint, arxiv:math/0103059

[GZ] L. Guo and B. Zhang, Renormalization of multiple zeta values, arxiv:math.NT/0606076 (2006).

[GSW] V. Guillemin, S. Sternberg, J. Weitsman, The Ehrart function for symbols, Surveys in Differential Geometry. Special memorial volume dedicated to S. S. Chern (2006). arXiv:math.CO/0601714v1.

[Ha] G. Hardy, Divergent series, Oxford University Press, 1967.

[H] M. Hoffman, Multiple harmonic series, Pacific J. Math. 152 (1992) 275-290; The algebra of multiple harmonic series, Journ. Algebra 194 (1997) 477-495, The Hopf algebra structure of multiple harmonic sums, arXiv:math.QA/0406589 (2004). 
[H2] M. Hoffman, Quasi-shuffle products, J. Algebraic Combin. 11, 49-68 (2000)

[Ka] Ch. Kassel, Quantum groups, Graduate Texts in Mathematics, 155, SpringerVerlag, New York, 1995.

[K] D. Kreimer, Chen's iterated integral represents the operator product expansion, Adv. Theo. Math. Phys.3 (1999)

[KSW1] Y. Karshon, S. Sternberg, J. Weitsman, The Euler-Maclaurin formula for simple integral polytopes, Proc. Natl. Acad. Sci. USA 100, no.2 (2003). 426-433.

[KSW2] Y. Karshon, S. Sternberg, J. Weitsman, Euler-MacLaurin with remainder for a simple integral polytope, Duke Math. Journ. 130, no.3 (2005) 401-434.

[KV] M. Kontsevich, S. Vishik, Determinants of elliptic pseudo-differential operators, Max Planck Institut preprint, 1994.

[L] M. Lesch, On the non-commutative residue for pseudo-differential operators with log-polyhomogeneous symbols, Ann. of Global Anal. and Geom. 17 (1998) 151187.

[M] D. Manchon, Hopf algebras in renormalisation, Handbook of Algebra, Vol. 5 (M. Hazewinkel ed.), 365-427 (2008). See also arXiv:math. QA/0408405.

[Mi] H.N. Minh, Des propriétés structurelles des polylogarithmes aux aspects algorithmiques des sommes harmoniques multiples, Notes de l'exposé du 12/01/06 au groupe de travail "Polylogarithmes et Polyzeta", Université Paris VII.

[MMP] Y. Maeda, D. Manchon, S. Paycha, Stokes' formulae on classical symbol valued forms and applications, preprint arXiv:math.DG/0510454 (2005).

[MP] D. Manchon, S. Paycha, Shuffle relations for regularised integrals of symbols Comm. Math. Phys 270 (2007) 13-31

[P] S. Paycha, From heat-operators to anomalies; a walk through various regularization techniques in mathematics and physics, Emmy Nöther Lectures, Göttingen, 2003 (http://www.math.uni-goettingen.de); Anomalies and regularisation techniques in mathematics an physics, Lecture Notes, Preprint, Colombia, 2003 (http://www.lma.univ-bpclermont.fr/ paycha/publications/html).

[PS] S. Paycha, S. Scott, A Laurent expansion for regularised integrals of holomorphic symbols, Geom. and Funct. Anal. (2) 17 (2007) 491-536

[W] M. Waldschmidt, Valeurs zeta mutiples. Une introduction. Journal de Théorie des Nombres de Bordeaux 12 (2000) 581-595.

[Z] D. Zagier, Values of zeta functions and their applications, in "First European Congress of Mathematics", Vol. II, 497-512, Birkhaüser, 1994; Multizeta values (manuscript).

[Zh] J. Zhao, Analytic continuation of multiple zeta functions, Proc. Amer. Math. Soc. 128, no.5, 1275-1283 (1999).

[Zu] V.V. Zudilin, Algebraic relations for multiple zeta values, Russ. Math. Surveys 58:1 (2003) 3-32. 